\title{
Integration of Neonatal Mortality Prediction Models into a Clinical Decision Support System
}

\author{
by \\ Hasmik Martirosyan, B.Sc.
}
A thesis submitted to the Faculty of Graduate and Postdoctoral Affairs in partial fulfillment of the requirements for the degree of Master of Applied Science
in
Biomedical Engineering

Ottawa - Carleton Institute for Biomedical Engineering (OCIBME)

Carleton University

Ottawa, Ontario

(C) 2015

Hasmik Martirosyan 


\begin{abstract}
This thesis describes the development of neonatal mortality risk estimation models using Artificial Neural Networks (ANNs), the integration of these models into the Physician-Parent Decision Support (PPADS) tool, and the pilot study to test the PPADS tool.
\end{abstract}

A set of data mining programs were created to automate the data preparation, the development of ANN models and the selection of models that satisfy the usefulness criteria specified by our clinician partners. These programs were used to classify neonatal mortality data ( $6 \%$ mortality rate) with the average sensitivity and specificity of $81 \%$ and $98 \%$ respectively.

The mortality models were integrated with the PPADS tool to provide predictions about the risk of mortality for neonates admitted to the Neonatal Intensive Care Unit (NICU).

The observational and survey study conducted with parents whose infant did not graduate (died) from the NICU gave encouraging results regarding the usefulness of the PPADS tool. 


\section{Acknowledgements}

First and foremost, I would like to thank my thesis supervisor, Dr. Monique Frize, for her support, encouragement and guidance throughout my graduate studies. I am very grateful to have had the opportunity to work with Dr. Frize.

Thank you to my co-supervisor, Dr. Jeff Gilchrist, who always made himself available to answer my questions, provided rich comments and suggestions, which were invaluable for the successful completion of my thesis work.

Thank you to my co-supervisor, Dr. Erika Bariciak at the Children's Hospital of Eastern Ontario, for her help, and for the information provided for this work.

I would like to thank my fellow graduate students, Ruqia Nur and Daphne Ong, for their knowledge and friendship.

Last but not least, I am grateful to my family for their love, support and encouragement. 


\section{Table of Contents}

\section{Contents}

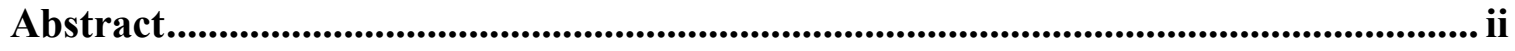

Acknowledgements ......................................................................................................................... iii

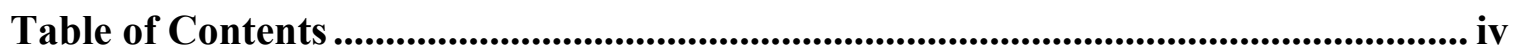

List of Tables ............................................................................................................ vi

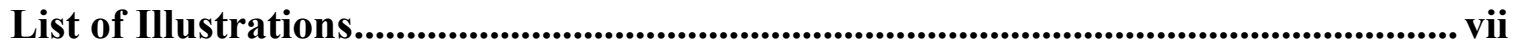

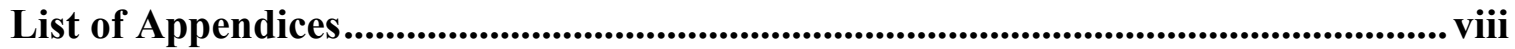

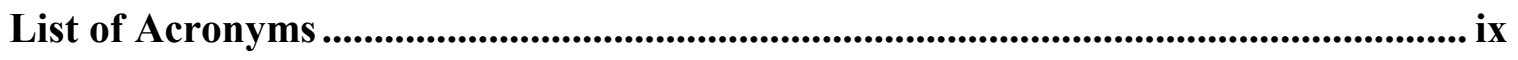

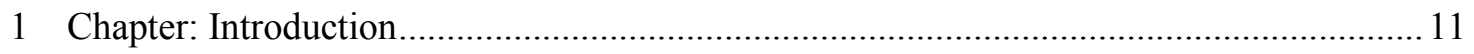

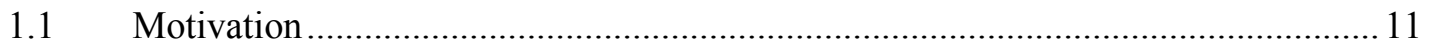

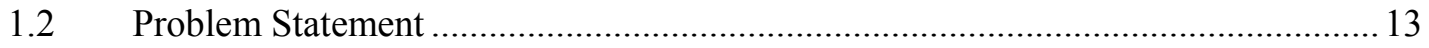

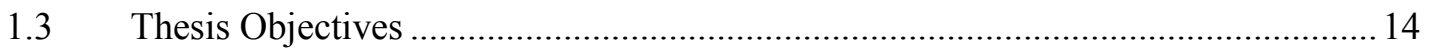

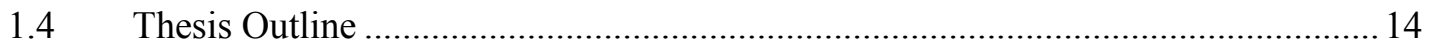

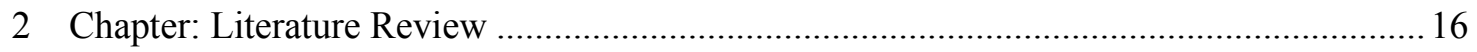

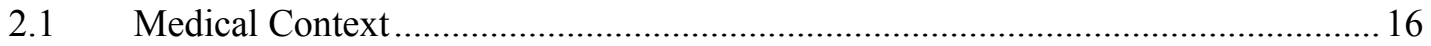

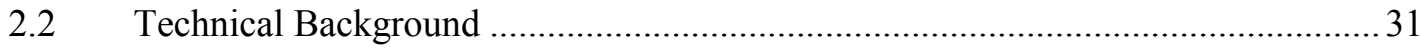

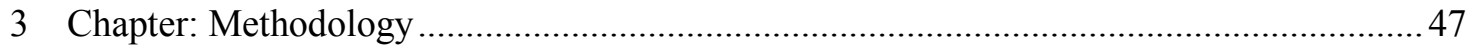

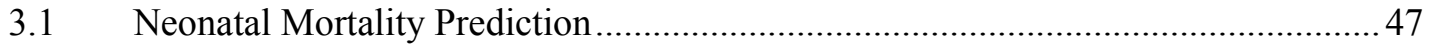

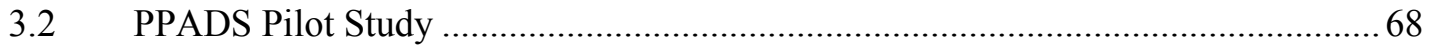

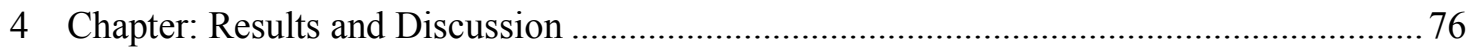

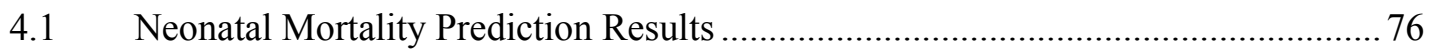

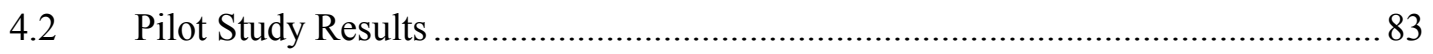

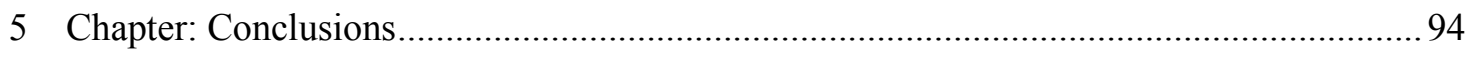




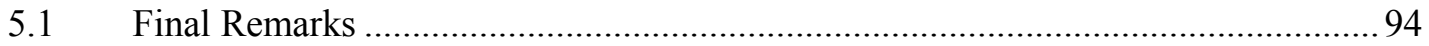

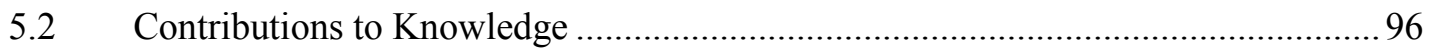

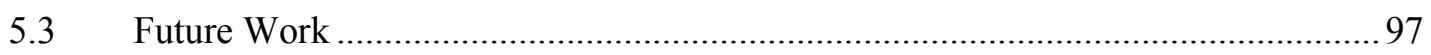

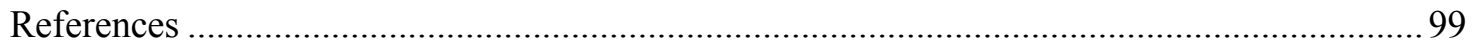

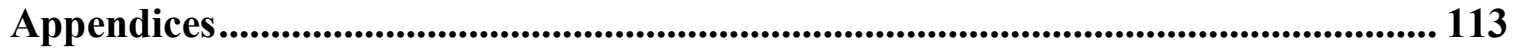




\section{List of Tables}

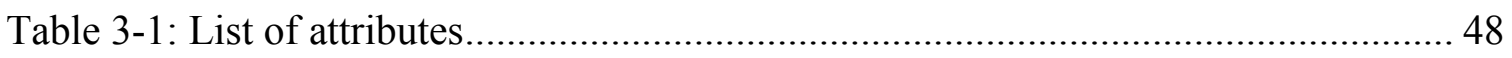

Table 3-2: Input File Format for the FANN library................................................... 51

Table 3-4: List of the options and parameters tested by the MIRG ANN Builder ........... 55

Table 4-1: Results of the classification of 12 hour summation data............................... 77

Table 4-2: Results of the classification of 48 hour summation data.............................. 78

Table 4-3: Results of the classification of 48 hour summation data with 3 attributes...... 79

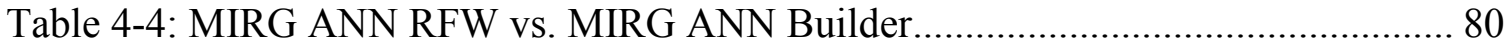

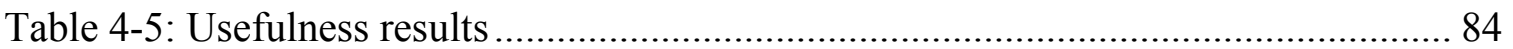

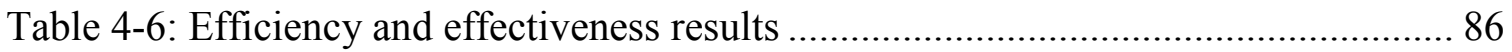

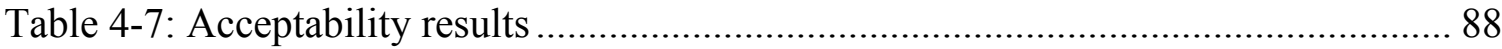

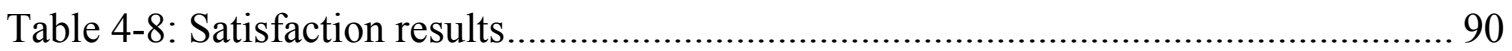

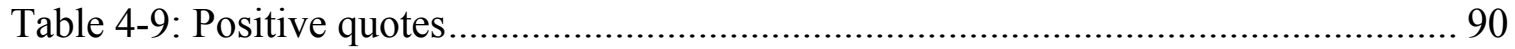

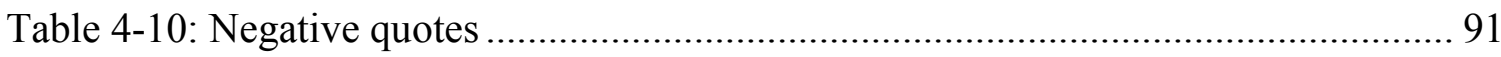

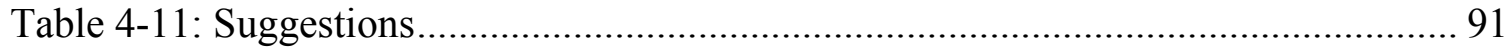

Table A-1: Description of the types of neonates admitted to the NICU....................... 113

Table A-2: Common Medical Conditions in the NICU ............................................ 113

Table A-3: Common Medical Therapies in the NICU............................................... 115 


\section{List of Illustrations}

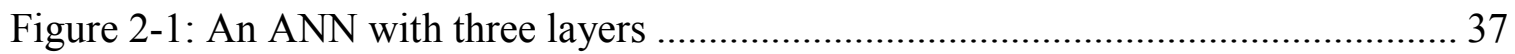

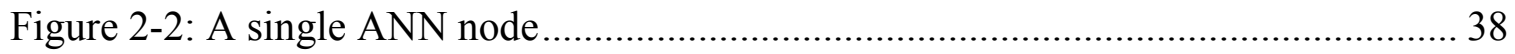

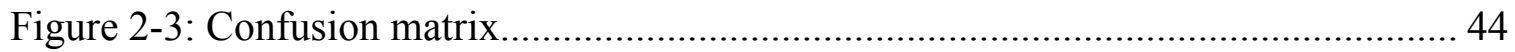

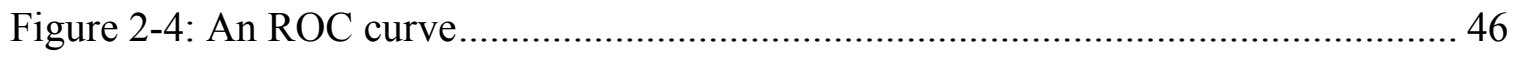

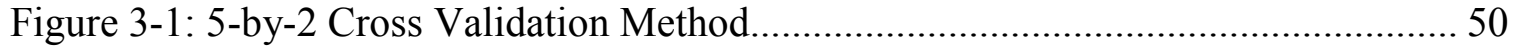

Figure 3-2: Flow Chart: MIRG ANN Builder. ...................................................... 56

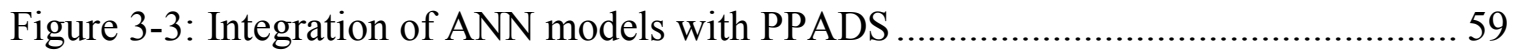

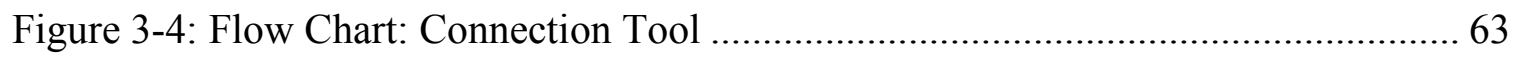

Figure 3-5: PPADS - Current Condition................................................................. 71

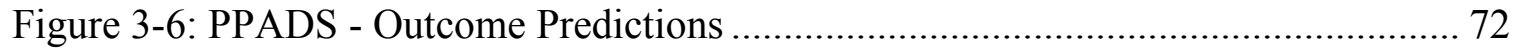

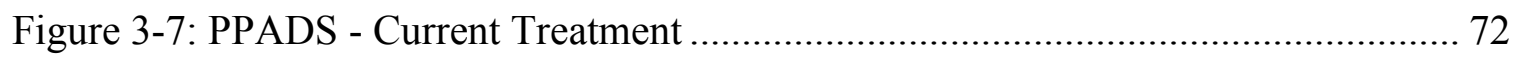

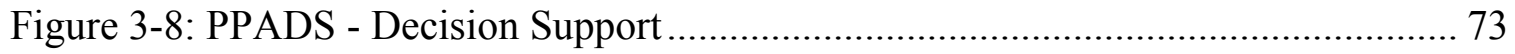

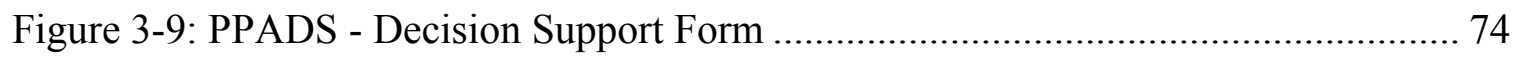

Figure 4-1: The patients_predictions table in the CDSS database ............................... 81

Figure 4-2: The predictions_info table in the CDSS database...................................... 81

Figure B-1: The first half of the result file created by the MIRG ANN Builder ............ 116

Figure B-2: The second half of the result file created by the MIRG ANN Builder ....... 117 


\section{List of Appendices}

Appendices.

Appendix A: NICU Environment.

Appendix B: Result File.

Appendix C: Ethics Clearance........................................118

Appendix D: Pilot Study Documents..................................122

D.1 Recruitment Letter..................................... 122

D.2 Study Test Participant Script............................ 124

D.3 Information Sheet and Parent Consent Form...................127

D.4 Feuillet de Consentement Parental............................130

D.5 Parental Background Questionnaire for the PPADS Tool.........131

D.6 Parental Evaluation Tasks for the PPADS Tool.................136

D.7 Parental Evaluation Questionnaire for the PPADS Tool..........139 


\section{List of Acronyms}

$\mathrm{ACC}$

ANN

AUC

BASH

BP

BPD

CBR

CDR

CDSS

CIHI

CHEO

CPR

CSV

EAV

ELBW

FANN

FN

FP

HRC

HeRO

IVH
Accuracy

Artificial Neural Network

Area Under Curve

Bourne-Again Shell

Blood Pressure

Bronchopulmonary dysplasia

Case-Based Reasoning

Clinical Data Repository

Clinical Decision Support System

Canadian Institute for Health Information

Children's Hospital of Eastern Ontario

Cardiopulmonary Resuscitation

Comma Separated Value

Entity Attribute Value

Extremely Low Birth Weight

Fast Artificial Neural Network

False Negative

False Positive

Heart Rate Characteristics

Heart Rate Observation system

Intraventricular Hemorrhage 


\begin{tabular}{ll} 
k-NN & k-Nearest Neighbor \\
MCC & Matthews Correlation Coefficient \\
MIRG & Medical Information technologies Research Group \\
NEC & Necrotizing enterocolitis \\
NICU & Neonatal Intensive Care Unit \\
NPV & Negative Predictive Value \\
PDA & Patent ductus arteriosus \\
PPADS & Physician PArent Decision Support \\
PPV & Positive Predictive Value \\
PVL & Periventricular leukomalacia \\
REALTROMINS & Real-Time Risk of Mortality and Instability \\
RFW & Research Framework \\
ROC & Receiver Operating Characteristic \\
SQL & Structured Query Language \\
TN & True Negative \\
TP & True Positive \\
VLBW & Very Low Birth Weight \\
\hline
\end{tabular}




\section{Chapter: Introduction}

\subsection{Motivation}

\section{Healthcare Perspective}

The Neonatal Intensive Care Unit (NICU) is a specialized hospital unit for managing and treating newborns with critical conditions. Many of the NICU infants are born prematurely and some of them may be at the limit of viability requiring a prolonged hospitalization involving invasive life support therapy. Due to technological advances it has become possible to sustain the life of these infants; however that has led to increase in the rate of the morbidity and the length of stay for non-survivors (Blackburn, 1982), (Safran, 2003), (Meadow et al., 2004). These trends in the neonatal care are raising questions regarding the appropriateness of certain interventions and are emphasizing the importance of informed and ethical decision-making (Bunch, 2002), (Anand et al., 2007).

Decisions that are being made by the healthcare team about patient management and resource allocation are based on clinical experience and intuition, outcome statistics specific to their institution, and information published in the medical literature (Frize et al., 2013). Clinicians try to predict the infant's outcome, including survival and potential complications, and determine the appropriate course of treatment. Based on these predictions, parents of these infants are counseled about various treatment options for their baby; the involvement of parents (or guardians) is important as they know the values of the family and the environment in which the infant will be raised (Tyson, 1995). 
However, parent participation in the decision-making can be overwhelming and confusing. Researchers have shown that parents often turn to the Internet to augment the information provided by the healthcare team; yet, the information they gather may not be applicable to their infant (Brazy et al., 2001), (Philips and Tooley, 2004).

The computerized decision support tools can address the gaps in the NICU decision-making by providing reliable information and outcome predictions to aid parents in making informed decisions with clinicians (Safran, 2003), (Frize et al., 2013).

\section{Engineering Perspective}

The Medical-Information technology Research Group (MIRG) has been actively involved in the development of Clinical Decision Support Systems (CDSSs) for health care, including neonatal intensive care, for many years. Several data mining tools for analyzing data and creating prediction models for various medical outcomes have been explored and tested by former students and the principal investigators.

MIRG has identified Artificial Neural Networks (ANNs) as a good pattern classification approach for medical data. Using MATLAB's Neural Network Toolbox as a base, Colleen Ennett developed an automated tool to fine-tune the parameters of feedforward backpropagation ANNs and applied the tool for the classification of the adult intensive care unit, the NICU and coronary surgery patient data (Ennett et al., 2004). The tool was later improved by Dawn Rybchynski to perform sequential experiments with increasing number of hidden nodes in a faster operational speed (Rybchynski, 2005). Lan Yang worked on an early conceptual architecture of a decision support tool for the NICU and developed a prototype using Visual Basic and Microsoft Access (Frize et al., 2005). 
This prototype was evaluated by physicians at the Children's Hospital of Eastern Ontario (CHEO) in a usability study. Physicians expressed their interest in the further development of the decision support tool. An early prototype of a combined CDSS and parent decision support tool, called Physician-PArent Decision Support (PPADS), was

developed and tested through a usability study by Sabine Weyand (Weyand, 2011). Daphne Townsend worked on creation of ANN models to predict the NICU outcomes such as the risk of mortality and various diseases using archived data (Townsend, 2007). Jeff Gilchrist developed a clinical data repository (CDR) with real-time data acquisition, storage, and retrieval in the NICU at CHEO, and created real-time risk prediction models using Decision Trees (DTs) (Gilchrist et al., 2011), (Gilchrist, 2012).

\subsection{Problem Statement}

The outcome predictions that are being displayed on the interfaces of the PPADS system have been manually added in order complete the prototype. Ultimately, the system will be integrated in the NICU to provide real-time predictions for the patients in

the NICU. Therefore, prediction models need to be combined with the PPADS system to provide clinically useful risk assessments in real-time (within one minute). Furthermore, the models and the ANN application by which the models have been created need to be flexible for the integration with different software environments.

In the previous works of our group, clinically useful ANN models were created using a MATLAB based tool. However, to make risk estimations for patients, data need to be passed to the prediction tool manually for each patient making the system not 
applicable for real-time risk estimation, and the MATLAB tool requires a commercial license for use.

This thesis describes further improvement of the PPADS system which involves the development of an automated ANN application, based on an open source library, to create neonatal mortality models that can be integrated into the PPADS tool to provide real-time risk predictions.

\subsection{Thesis Objectives}

The first objective of this thesis is to improve mortality prediction models making these suitable for the real-time risk assessment. For these purposes, ANN libraries have been explored and compared to create an ANN application that has a fast and automated performance.

The second objective of this thesis is to conduct a short pilot study involving parents whose child has not graduated (not survived) from the neonatal intensive care unit.

\subsection{Thesis Outline}

This thesis is divided into the following chapters:

Chapter 1 has presented the motivation, problem statement and thesis objectives.

Chapter 2 is divided into two parts: First, a medical context covering a detailed review on the NICU environment, decision making and current decision aids in the NICU. The second part is the technical background, which provides the relevant concepts for databases and data mining methods. 
Chapter 3 details the methodologies used in creation of prediction models and integration as well as the methods used in conducting the pilot study.

Chapter 4 presents results and the discussion of the results.

Chapter 5 presents conclusions, contributions to knowledge, and future work. 


\section{Chapter: Literature Review}

\subsection{Medical Context}

\subsubsection{NICU environment}

The NICU provides care to prematurely born infants and full-term newborns with medical complications. The NICU team consists of a multidisciplinary healthcare team involving neonatologists and nurses with the addition of dieticians, pharmacists, respiratory therapists, pediatric subspecialists and others.

Neonates admitted to the NICU belong to the following categories: Premature neonates (born before 37 weeks of gestation), lower than normal birth weight neonates (weighing less than 2500g), and high risk neonates (neonates with congenital abnormalities, diseases or other complications) (Yee et al., 2006). A detailed description of the types of neonates admitted to the NICU can be found in Appendix A.

According to the Canadian Institute for Health Information (CIHI) data, there has been an increase in the number of infants admitted to the NICU over the past years. In $2003-2004,13.6 \%$ of newborns required care in the NICU, whereas it was $12.6 \%$ in 1994 -1995 (CIHI, 2006). This trend can be attributed to an increase in the number of prematurely born babies: Premature babies make up the majority of the infants in the NICU and the rate of preterm birth has increased in Canada by $0.6 \%$ since 1995 , reaching $7.6 \%$ by the year 2002 (Joseph et al., 2007).

Since the care in the NICU has improved due to advancements in technology, the survival of neonates has increased as well: Compared to survival rates for preterm born 
neonates in early 1970 's, which was under $10 \%$, today over $90 \%$ of babies born at 28 weeks survive. However, this improvement has led to higher health care costs, since the more premature a baby is born, the longer this patient must stay in the NICU, thus increasing the cost of neonatal intensive care (Lantos and Meadow, 2011). As an example, in 2005-2006 the average in-hospital cost in Canada for a normal birth weight infant (2500 grams or more) was $\$ 1,084$, whereas the cost for a low birth weight infant was $\$ 12,354$ (CIHI, 2009).

\subsubsection{NICU outcomes and trends}

According to the physicians working in the NICU at CHEO the most common medical complications among the NICU patients include sepsis, respiratory distress syndrome (RDS), intraventricular hemorrhage (IVH), seizures, apnea, periventricular leukomalacia (PVL), pneumothorax, bronchopulmonary dysplasia (BPD), patent ductus arteriosus (PDA), and necrotizing enterocolitis (NEC). A few of the most common therapies used in the NICU are artificial ventilation, artificial nutrition, antibiotic therapy, catheter drainage, intravenous therapy, chest tube drainage, and blood product transfusions (Weyand, 2011). A brief description of each of the medical conditions and therapies mentioned above can be found in the Appendix A.

Outcomes for the infants in the NICU include death, survival with disability (morbidity) and intact survival (minor or no disability) (Lantos and Meadow, 2011). Research has shown that infant mortality rates are highly affected by low birth weight and shortened gestational age (Mohamed et al., 2010), (Allen et al., 1993). Through their analysis of the National Inpatient Sample Database and its pediatrics-only sub-portion for 
the years 1997-2004, Mohammed et al (2010) reported that the overall survival rate for infants with a birth weight of $500 \mathrm{~g}$ was $8 \%$, whereas those who lived through the first 3 days of life had a chance of survival of up to $50 \%$ (Mohamed et al., 2010). The authors also showed that the survival rate of infants in the weight group of 500 to $749 \mathrm{~g}$ was $50 \%$ and increased to $70 \%$ if they survived through the third day, and reached to $80 \%$ by the end of the first week. The study conducted by Allen et al (1993) on 142 infants born at the Johns Hopkins Hospital in Baltimore, from 1988 to 1991, showed that there was a decrease in survival rate with decreasing gestational age. Another trend in the NICU shown by the study was the decreased probability of survival with no abnormalities with lower gestational age (Allen et al., 1993).

The survival rate for infants in the NICU who are born with an extremely low birth weight (ELBW) at the age of viability (22-23 weeks gestation) increased over the past few decades because of technological advances and the collaborative efforts of obstetricians and neonatologists (Goldenberg et al., 2008). A study conducted by Horbar et al (2012) on 806 infants born between 2000 - 2009 in North American hospitals with various birth weights showed that mortality for infants weighing 501 to $1500 \mathrm{~g}$ decreased from $14.3 \%$ to $12.4 \%$ from 2000 to 2009 . There was also a decrease, from $46.4 \%$ to $41.4 \%$ in major morbidity among survivors. For infants born in 2009 , the mortality rate was $36.6 \%$ in the 501 to $750 \mathrm{~g}$ weight category and $3.5 \%$ for infants weighting from 1251 to $1500 \mathrm{~g}$, whereas major morbidity in survivors was $82.7 \%$ and $18.7 \%$ for the same weight groups. In $2009,89.2 \%$ of infants in the 501 to $750 \mathrm{~g}$ weight category and $49.2 \%$ of all very-low-birth-weight (VLBW) infants either died or survived with a major neonatal morbidity (Horbar et al., 2012). 
Infants who survive remain at risk of developing a wide array of complications, not only while in the neonatal unit, but also in a long term (Saigal and Doyle, 2008). In their study of 242 survivors among VLBW infants and 233 normal birth weight infants, Hack et al (2002) found that fewer VLBW young adults than normal-birth-weight young adults had graduated from high school (74\% vs. 83\%) (Hack et al., 2002). Saigal and Doyle (2008) showed that teenagers born with extremely low birth-weight (ELBW) function significantly worse intellectually and on other achievement measures compared to higher birth weight babies (Saigal and Doyle, 2008). The study also showed that three achievement scores (reading, spelling and arithmetic) of teenagers who had a birth weight of less than $750 \mathrm{~g}$ was less than half of the normal range.

\subsubsection{Decision making in the NICU}

During an infant's stay in the NICU, there are many important decisions that need to be made such as type of medications to administer, the need for surgeries, level of intensive care to implement, and possibly change in the direction of care. The latter is one of the most difficult care decisions, where physician and parents decide between four main options: full active care (continuing and adding all available treatments), nonescalation of care (continuing treatments that are currently in place and not adding new treatments), do not resuscitate order (not providing a full resuscitation in the event of a severe heart or lung deterioration), and withdrawal of life support (removing invasive and painful life support technology).

These changes in the direction of care options are not uncommon in the NICU as numerous researchers have shown. In their retrospective study Ryan et al (1993) 
analyzed the records of infants who died in the NICU at the University of Alberta Hospitals between January 1990 and December 1991. Their results show that when excluding deaths resulting from brain death and failed cardiopulmonary resuscitation (CPR), $80 \%$ of all deaths were a result of withdrawal of therapy and $20 \%$ were no-CPR orders (Ryan et al., 1993).

Weiner et al (2011) found similar trends when quantifying modes of death for hospitalized infants from year 1999 to 2008 at Children's Mercy hospital in Kansas City: Out of 414 death cases $61.6 \%$ had withdrawn care, $20.8 \%$ had withheld care, and $17.6 \%$ received CPR (Weiner et al., 2011).

\subsubsection{Ethics in the NICU}

As there is much uncertainty involved in the NICU outcomes and it is not always clear whether treatment will result in an acceptable quality of life, there are difficult ethical challenges involved in making decision such withdrawing or withholding therapies. There are generally four main principles in the ethical decision making in the NICU (Tyson, 1995), (Becker and Grunwald, 2000).

1. Beneficence - doing most good possible to preserve life and reduce pain of the infant.

2. Nonmaleficence - doing no harm or at least minimal harm.

3. Justice - treating all infants fairly and giving all an equal chance. 
4. Autonomy - respecting the best interest of the infant. As an infant cannot make decisions for itself autonomy is passed to principal decision makers: parents or guardians.

The decisions that parents and health-care professionals make need be balanced between best interest (beneficence) of the infant and minimizing harm to the infant (nonmaleficence) (Cloherty et al., 2008).

\subsubsection{Shared decision making in the NICU}

Shared decision-making is being more widely accepted in neonatal care to provide ethical and best possible care to the infant. In the NICU environment physicians have experience, medical and healthcare knowledge, and parents (or guardians) know best the values of the family culture and the environment in which the infant will be raised. In shared decision making, physicians and parents work together to make healthcare choices, where values and best available evidence are combined to reach a high quality decision (Coulter and Collins, 2011), (Stacey et al., 2008).

\subsubsection{Decision making conflicts}

It is important for both, parents and physicians to share information and decision making responsibility for the shared-decision making to take place (Coulter and Collins, 2011). The NICU environment is complex and parents often feel overwhelmed, emotionally distressed, and confused. As a result, they often experience uncertainty and dissatisfaction with the decision making. There are often conflicts between physicians 
and parents about certain direction of care options. This is particularly true when a poor prognosis is predicted and care providers believe that withdrawal of life support is the best option, while the family wants to keep the infant alive at all costs. These conflicts result in frustration and distress for both the family and the medical team (Kopelman, 2006), (Eden et al., 2010), (Frize 2011, 50-51).

Kopelman (2006) studied several cases of such conflicts and argues that most conflicts result from insufficient time and effort taken by the medical team to fully understand the parents' reasons for requesting continued life support. The author suggests that adequate time must be found to understand a family's personal experience, reasoning, feelings, and religious and ethical beliefs in order for the physicians to support them and help them to make the best possible decisions for their critically ill infant (Kopelman, 2006).

In their study McHaffie et al (2001) explored parents' perception of treatment withdrawal or withholding by interviewing 108 parents from East Scotland whose infant died between 3 and 13 months prior to the interview. Parents identified full and honest information along with the evidence of a poor prognosis to be essential factors in minimizing doubt and guilt in decision making (McHaffie et al., 2001).

\subsubsection{Parents' decision aids}

In a busy clinical environment it is hard for a medical team to provide comprehensive information about the infant's condition, treatment options, and other medical information to parents to help them make informed decisions. To access information, parents often turn to other sources such as the Internet. In fact, Brazy et al 
(2001) showed in their study that more than half of parents of premature infants spend at least 20 hours a week to seek information. However the information, that parents obtain, may not be accurate or not applicable to their infant; this may confuse parents about what is happening in their particular case (Brazy et al., 2001).

To address these gaps, decision aids are being created and recommended for various situations in the NICU. These aids come in various forms such as: simple onepage sheets outlining the choices, or more detailed leaflets, or computerized decision aids (Schwartz, 2011).

To be reliable decision aids must be based on research evidence, present outcomes and risks in an understandable and unbiased way. A difference between a decision aid and traditional patient (or parent) information materials is that the latter tells patients what to do, but decision aids provide information to help them understand their options (Coulter and Collins, 2011).

\subsubsection{Current computerized decision aids for parents}

There are not many decision aids available for the NICU, and there are even less computerized ones. BabyCare link, a commercially available Internet-based telemedicine system, designed by researchers from Harvard Medical School and the Beth Israel Deaconess Medical Center, is the only computerized decision-aid for parents described in the literature (Gray et al., 2000).

The BabyCare link system focuses on VLBW infants in the NICU and is said to be designed to provide enhanced medical, informational, and emotional support to families as well as reduce the costs of care (Gray et al., 2000). Using BabyCare link, 
parents can monitor their infant from home, schedule visits, and find medical information about their infant's condition.

After a randomized study, Gray et al (2000) concluded that users of the tool report a higher quality of care and an enhanced interaction between families, staff, and community providers. However, the tool does not provide outcome predictions or any means of helping parents clarify their preferences or make decisions.

\subsubsection{CDSS for physicians}

Clinical decision support systems (CDSSs) that provide outcome predictions and prognosis are mainly developed for aiding physicians in decision making. CDSSs can be categorized into either a knowledge-based system or a non-knowledge-based system. Knowledge-based systems contain rules, guidelines, or compiled knowledge derived from the medical literature; most of the CDSS available to date involve this category. Non-knowledge based systems use artificial intelligence in the form of machine learning, to allow the computer to learn from past experiences, or analyze and detect patterns in medical data (Berner 2007, 4-8).

To develop a novel CDSS for neonatal care, our research group explored the

development of a non-knowledge based system versus knowledge-based system which are more often reported on. 


\subsubsection{CDSS for the NICU}

A few CDSSs for the NICU are described in the literature and those are presented here.

\section{$\underline{\text { Heart Rate Observation System (HeRO) }}$}

In their effort to improve outcomes for VLBW infants researchers at the University of Virginia have developed an observation system for the detection of abnormal heart rate characteristics (HRC) for the early detection of sepsis (Moorman et al., 2011). Using mathematical and statistical tools, the HeRO system analyses heart rate characteristics with time-domain parameters (mean and variance of the heart rate signal), frequency-domain parameters (band specific variance), phase domain (phase of waveforms), signal quality index, entropy estimation and deceleration detection using wavelet transform based algorithms. These are then combined through multivariate statistical methods such as logistic regression, k-nearest neighbor, and other techniques (Moorman et al., 2011). To confirm that HeRO improves neonatal outcomes, Moorman et al (2011) conducted a controlled clinical trial of 3003 VLBW infants in 9 neonatal intensive care units, between April 2004 and May 2010. Infants were divided into two groups: for one group, HRC monitoring was displayed, while the results were not displayed for the other group. Results indicated that there was a reduced mortality rate in VLBW infants, whose HRC monitoring was displayed, from $10.2 \%$ to $8.1 \%$, and there was a trend towards an increase for days alive and ventilator free: 2.3 days more compared to the control group (no HRC display) (Moorman et al., 2011b). 


\section{$\underline{\text { Artemis Analytic System }}$}

The Artemis Analytic System has been designed with the collaboration between the IBM T.J. Watson Research Center, The University of Ontario Institute of Technology, and The Hospital for Sick Children in Toronto where the prototype has been piloted. The system is intended to accomplish two goals: first, analyze physiological data in real time to discover relationships between medical data and medical conditions that lead to medical complications such as pneumothorax, IVH, and PVL; secondly, run clinical rules such as "notify me when an hourly systolic blood pressure (BP) reading exceeds 140.' to inform physicians about early indicators of these conditions (Blount et al., 2010).

The Artemis framework uses Capsule Tech Inc. DataCaptor for collecting physiological data streams of patients' such as the heart rate, respiratory rate and blood oxygen saturation. Data processing is accomplished by the IBM InfoSphere Stream Computing system along with the Stream Processing Language (SPL) programming language for programming and controlling the stream computing system. Knowledge extraction is performed using multidimensional, temporal and data stream data mining frameworks developed by the Artemis team (Blount et al., 2010), (Thommandram et al., 2014).

Thommandram et al (2014) employed the Artemis system to classify neonatal spells, which are cardiorespiratory events that occur in neonates with variable combinations of suspension of breathing, a fall in heart rate and decrease in blood oxygen saturation (Thommandram et al., 2014). To identify respiratory pauses the system detected breaths and the intervals between the breaths. Changes in heart rate and blood 
oxygen were identified using threshold breach detectors and algorithms that detect relative change events in individual signals. In order to classify neonatal spells the events detected in the individual signals were synchronized together and a rule-based classifier was applied. To evaluate the performance of event detection algorithms and the rulebased classifier a 24 hour sample of data from a neonatal patient, who was reported to be experiencing spells, was used. The authors compared the results of the algorithms to the manual detection of events by a neonatologist. The accuracies of detecting pauses in breathing, changes in heart rate and blood oxygen saturation were $98.9 \%, 97.8 \%$ and 98.3\% respectively. Neonatal spells were classified with $98.9 \%$ accuracy (Thommandram et al., 2014).

\section{$\underline{\text { REALTROMINS }}$}

The medical system REALTROMINS (REAL-Time Risk of Mortality and Instability), developed at the University of North Carolina at Chapel Hill, uses real-time predictive analytics to identify high risk patients in NICUs and Pediatric Intensive Care Units (PICUs) and present information to physicians through a user interface (REALTROMINS Inc, 2014).

The data that the REALTROMINS system collects consist of physiologic signals (e.g. ECG), physiologic based measures of organ function (e.g. serum glucose), demographic (e.g. age) and diagnosis related (e.g. post-operative status) predictors of mortality. Measures of variability for one or more physiological signals are calculated and analyzed to determine whether a particular variable is within a critical interval; if that is the case an occurrence of an event for a patient is recorded. Over a period of time such 
events are counted and presented to clinicians in graphic form to illustrate the risk of mortality. Data is also used to create mortality prediction models utilizing neural networks in SAS Enterprise Miner software. The models estimate the risk of mortality and the information is presented to physicians real-time with an update of every two minutes (Kocis and Kocis, 2012).

The pending patent application submitted by Kocis and Kocis (2012) presents the performance results of the REALTROMINS system (Kocis and Kocis, 2012). The data of 28 patients of the Pediatric Intensive Care Unit, half of whom died, were used to create mortality models with ANNs. The first four hours of the admission data of 28 patients were partitioned, creating 1680 "survive" packets and 1680 "died" packets: 3360 records in total; $80 \%$ of packets were used for the training and a random $20 \%$ hold out sample of packets for validating the network. The reported predictive capabilities of the model were $98.44 \%$ sensitivity and $98.88 \%$ specificity on the training set, with identical results for the validation set (Kocis and Kocis, 2012).

\section{NEONATE}

The NEONATE project was carried out by a research team at the University of Aberdeen, United Kingdom. The aim of the project was to investigate sub-optimal decision-making in the NICU and develop a CDSS which can alert physicians and nurses to situations where specific actions should be taken or avoided (Hunter et al., 2003).

Researchers tried to discover examples of sub-optimal behavior in the NICU by recording the actions performed on a patient with an accuracy of a few seconds and physiological parameters sampled every second. 
Clinical staff was interviewed to develop a lexicon for observations and actions. As a result, a lexicon of 51 terms for observations (e.g. Movement), and 191 for actions (e.g. Feeding) was generated. During the data collection, from October 2001 to February 2002, about 407 patient-hours of observations on 31 separate infants were recorded and data were stored in a Microsoft Access database. Then, using Time Series Workbench, data were synchronized: physiological measurements were linked to the observations and actions taken.

An example for an action requiring decision-making described by the authors is the manual ventilation of an infant. The protocol for carrying out this action was that transcutaneous oxygen reading was less than $3 \mathrm{mmHg}$ or oxygen saturation was less than $60 \%$ for at least 10 seconds, and the heart rate was less than 100 beats per minute. By looking at the data, the implemented protocol would generate false positives and negatives as well as true positives and negatives by comparing actual action taken by the clinical staff (considered always right) and what should have been done according to the protocol. Clinical experts then were supposed to comment on the appropriateness of actions, both actual and recommended; the assumption was that, through a refinement of the protocol, the recommended actions would be considered correct in some cases and actual actions would become subject to question leading to identification of suboptimal decisions. However, the researchers found that the clinical experts were reluctant to make recommendations on actions just by looking at the local information and individual actions; rather, they wanted to review more detailed patient history with preceding actions. The conclusion researchers reached was to take into account respiratory 
management as a whole when formalizing a protocol, making it much more complex to develop (Hunter et al., 2003).

\section{PPADS (Physician-Parent Decision Support)}

The CDSSs presented earlier in this section provide outcome estimates that can be viewed by clinicians, but, to our knowledge, none of them provides decision support or other information for facilitating parent decision-making.

To augment the shared decision-making in the NICU our research team at Carleton University developed the PPADS tool. It is a combined decision aid both for health care professionals and for parents to help make more informed difficult care decisions. The PPADS tool currently provides predictions for mortality and is expected to enhance the evidence upon which critical decisions are made in the NICU (Frize et al., 2011).

The PPADS tool, developed using the Drupal content management system, consists of two distinct user interfaces: a clinician and a parent interface for patients in the NICU. The parent interface provides necessary information about the infant along with risk estimations, and provides a decision support module that helps them clarify and communicate their personal values associated with the different aspects of options available to them. The clinician interface contains the list of all patients with corresponding files of admission and various medical details including outcome predictions (Weyand, 2011).

The first phase of the usability study, involving parents who had a neonate who graduated (survived) from the NICU, was conducted by our group. Eight parents, who 
participated in the study, found the tool easy to use and completed the required tasks without any miss-clicks or system navigation errors. Furthermore, the participants indicated they would use the tool if given the opportunity and provided rich comments on how to improve the tool; adding more information was the most common suggestion (Frize et al., 2011), (Frize et al., 2013).

\subsection{Technical Background}

\subsubsection{The data}

The data used in this work are collected and stored by the Clinical Data Repository (CDR) at CHEO designed by our research group using open-source tools (Gilchrist et al., 2010), (Gilchrist et al., 2011b). The CDR uses the Entity-Attribute-Value (EAV) format and collects data in real-time from monitors, ventilators, pulse oximeters, laboratory data, admission and discharge information, and discharge diagnoses. It was designed to accommodate a fast-changing medical environment and supports the addition of new attributes automatically. To protect patients' private information, confidential data is split from raw research data before it is stored, which enables researchers and physicians to access real-time data without violating patient privacy; a study ID is used to associate the patient with the research data in an anonymous way (Gilchrist et al., 2011b), (Gilchrist, 2012).

The EAV format that the CDR uses is able to provide a flexible and simple schema for collecting biomedical data, storing it in one generic table with three columns: entity, attribute, and value (Gilchrist et al., 2011b). Additionally, to incorporate the time 
at which the value was measured, the CDR has a fourth column for the Entity-AttributeValue-Time. The CDR design allows the use of multiple data types in a single table, making it a hybrid format, which provides simpler and faster queries, and the ability to use multiple data types without having to first convert the data. As NULL values require very little memory, these are used in the columns which are not used. Gilchrist et al (2011) tested this design for storage capacity, query speed, and query complexity, and compared to the other EAV formats such as the simple and the multi-data EAV (Gilchrist et al., 2011b). For the storage performance of 5.5 million points of data, the hybrid EAV format used the least amount of space at $414 \mathrm{MB}$, the simple EAV format used $417 \mathrm{MB}$ of data, and the multi-data type format used the most space at 421MB. For the query speed, the time taken for querying 282,584 data points from one patient was $2.87 \mathrm{~s}$. for the simple EAV, $2.81 \mathrm{~s}$. for the hybrid EAV format, and $8.44 \mathrm{~s}$. for the multi-data type format. And lastly, for handling query complexity, the simple EAV format needed only 9 data elements, the hybrid EAV needed 11 elements due to the fact that it has separate columns for each data type, and the multi-data type query was the most complex, needing 39 elements. Researchers concluded that, under the conditions tested, the hybrid EAV approach provided a nice balance between the simple and the multi-data type formats (Gilchrist et al., 2011b).

Furthermore, to test the perceived usefulness of the CDR prototype in a hospital setting, a usability study was conducted at CHEO by our research group (Ong et al., 2013). A secure online survey was distributed to 209 physicians, 125 research institute investigators, and 35 research institute coordinators at CHEO through email; the team was hoping to secure at least 55 responses, but 88 replied (63 fully and 25 partially 
completing the survey). According to the survey responses, the majority of participants felt the CDR was a useful tool, showed interest in it, and thought it would be important to have such a system in the future work.

\subsubsection{Handling missing data}

Medical databases often contain missing values, and finding an approach to impute these values is an important part of the data preparation process. This is true particularly for the ANNs, since they are not able to interpret missing values and cannot successfully classify the outcomes if missing values are present in the data (Ennett et al., 2001).

Several approaches can be used to deal with missing values. One option is to delete the cases with many missing values for the variables under consideration. However, this approach can result in the loss of potentially valuable information. Another approach is to replace all missing values with the averages, but there is a negative side to this approach; data may become biased towards sick patients, since they are likely to have more tests than less sick infants. Another technique used to deal with missing values is to replace these with normal values; however, determining normal values for infants is a difficult task, since there are many factors affecting the different parameters such as the birth weight and the gestational age. Moreover, this approach would bias the results towards less sick infants (Ennett et al., 2001).

Our research group uses another technique, a Case-Based-Reasoning (CBR) system with k-Nearest Neighbor (k-NN) algorithm to find an average value for similar cases, to impute missing values (Ennett et al., 2008). Haque et al (2000) describe the 
CBR as a "computer technique, which combines the knowledge based support philosophy with a simulation of human reasoning when past experience is used, i.e. mentally searching for similar situations that happened in the past and reusing the experience gained in those situations" (Haque et al., 2000). In the case of the CBR, the structured and stored knowledge is queried from databases to solve a problem (Haque et al., 2000).

There are four main steps involved in the CBR cycle: Retrieving the most similar cases for the case under consideration, reusing those cases to attempt to solve a problem, revising the proposed solution if necessary, and retaining the new solution as a new case in the database (Watson and Marir, 1994). From this cycle, the CBR automatically retrieves and reuses cases to input missing values in databases (Frize and Walker, 2000), (Ennett et al., 2001). The retrieval of cases in the CBR is performed by the k-NN algorithm (Rybchynski, 2005). The latter, ranks the cases by the similarity to the current case and uses the parameter $\mathrm{k}$ to determine how many cases to retrieve. The k-NN distance algorithms plots all points in the k-dimensional space. Then, the distance from the target case to all other cases in the case-base is calculated: The cases with the smallest distance to the target case are chosen as the most similar. In a weighted k-NN algorithm, the axes of k-spaces are not uniformly set: The input variables with higher weights are plotted on a new, elongated axis, whereas the variables with lower weights are plotted on a shrunken axis. Consequently, the difference in the values along the axis becomes more important for the input variables with higher weights, and less important for the variables with lower weights (Rybchynski, 2005). The variables with a missing value are not drawn on the axis for that particular case, but the distance is still calculated; this is one of the strengths of the k-NN algorithm in dealing with missing values. However, as a 
weighted algorithm, the k-NN has a weakness: a predefined knowledge of the weights for the inputs is required. Typically, uniform weights are used (all weights are equal). The other options are the expert-chosen weights, or the weights identified by using complete cases submitted to an ANN (Ennett, 2003), (Ennett et al., 2008), (Gilchrist, 2012).

In the CBR system that our research group is using, the k-NN algorithms uses user defined weights and the parameter "k" was arbitrarily chosen to be 10: For imputing a missing value, 10 closest matching cases of an incomplete case are retrieved and the missing value is replaced by the mean value of those 10 cases (Rybchynski, 2005).

\subsubsection{Normalizing the data}

Data normalization can be performed in order to eliminate the effect of the range differences of different attributes, and to reduce the training time of the ANNs (Townsend, 2007). The modified Z-score transformation (Equation 2-1) can be used to perform the normalization.

$z=\frac{x-\mu}{3 \sigma} \quad$ [Equation 2-1]

Where the variable to normalize is the $\mathrm{x}, \mu$ and $\sigma$ are the average and the standard deviation for the variable $x$, and $z$ is the normalized value.

\subsubsection{Outcome prediction}

\subsubsection{ANNs - An overview}

Artificial Neural Networks (ANNs) can be defined as collections of interconnected elements, called nodes or neurons, which can learn to recognize patterns: 
these are abstract models inspired by the knowledge of the brain's function. The weights on the connections contain the knowledge of a network. Each node mathematically combines the inputs it receives to determine if it will activate, and so affect other nodes it is connected to (Kahn, 1994).

An ANN is trained by presenting it with a set of input and output variables. Training the ANN gives weights to its connections, and these can be applied to classify new and unknown cases (Kahn, 1994). To test the reliability an ANN model, data used for the classification are usually divided into two sets. The first set, referred to as the training set, consists of cases used for the learning process through which the ANN learns and modifies itself. The second set, called test set, consist of cases previously unknown to the trained ANN: These cases are used to do the classification and compare the results to actual class values. Additionally, a verification set, again consisting of cases previously not seen by the ANN, can be used to test it, improving the confidence in the results (Rybchynski, 2005). In theory, if the training set is large and diverse enough, ANNs can accumulate enough knowledge to classify unknown cases reliably (Kahn, 1994).

A large advantage of using ANNs is their ability to learn directly from observations; they do not require rules or other knowledge from a domain expert. The greatest disadvantage of ANNs is their inability to produce meaningful explanations for their decisions; the reasoning is contained within the numerical values of the weights. This may result in difficult acceptance of ANNs among physicians as they prefer to know the basis for the system's decision before accepting and acting on its advice (Kahn, 1994), (Berner 2007, 4-8). 


\subsubsection{ANN components and learning}

The architecture of an ANN is defined by its structure and connectivity. The number of hidden layers and nodes in each layer make up the structure of the network. As for the connectivity, the network can be either fully or sparsely connected. In the fully connected networks each node in the layer is connected to every node, both in the next and previous layers, whereas in the sparsely connected networks there can be any number of connections of the nodes across the layers. There are weights assigned to each connection, defining how strongly a particular input will affect the node it is connected to (Rybchynski, 2005). An example of a multilayer ANN is shown in Figure 2-1.

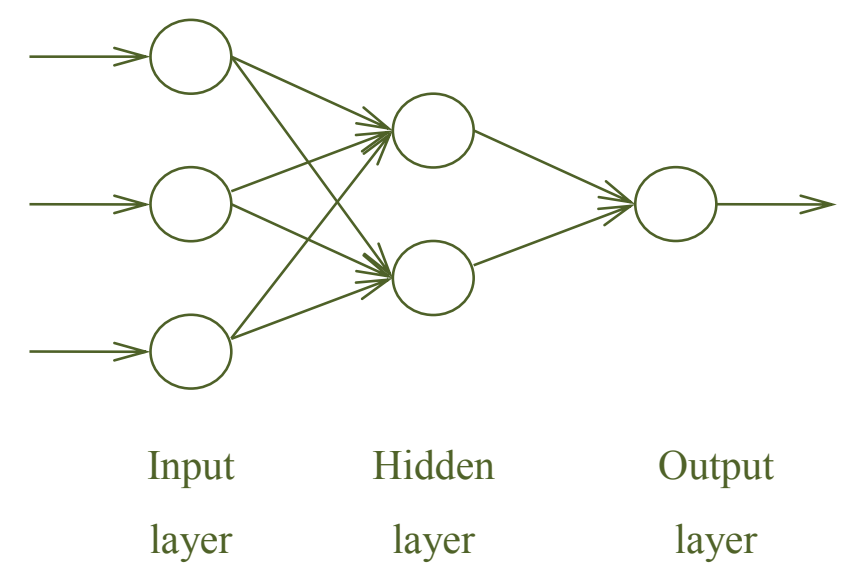

Figure 2-1: An ANN with three layers

Data processing within ANNs is accomplished by the propagation, the activation (also called transfer) and the output functions as illustrated in Figure 2-2 (Kriesel 2007, 33-38).

When the ANN receives the outputs of other nodes, the propagation function, using the weights of the connections, transforms these outputs into an input for the receiving node: the weighted sum is a common choice for a propagation function. 


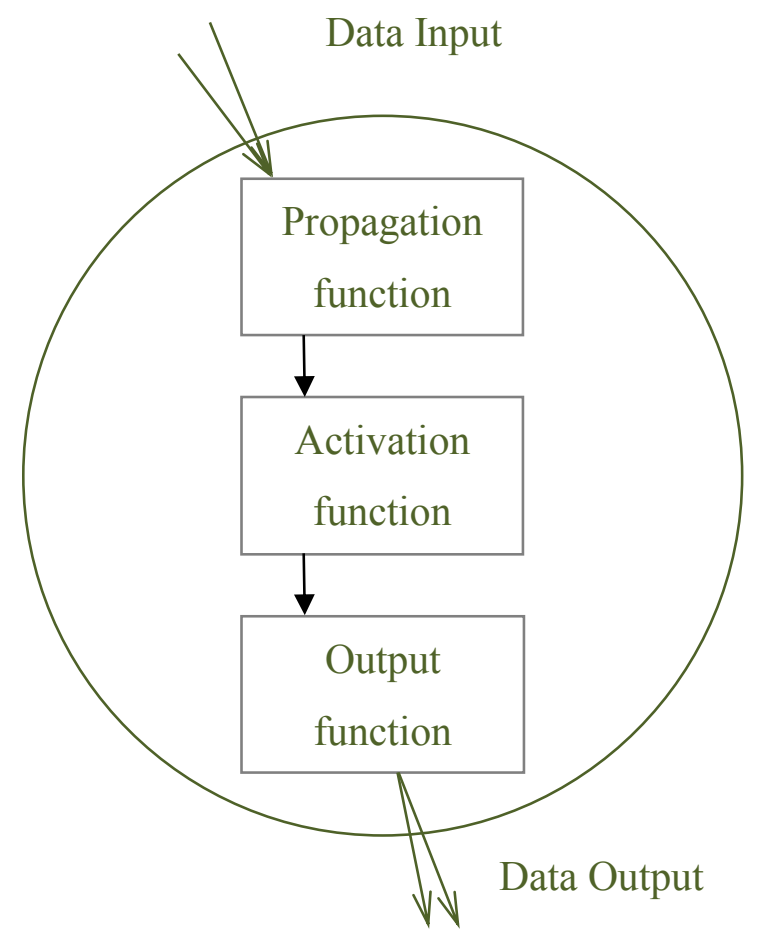

Figure 2-2: A single ANN node

Next, the activation function takes this input, and using a threshold determines whether the node will activate or not, and also the extent of the activation. The threshold is usually included in the definition of the activation function. Commonly used activation functions are the mapping functions, such as the hyperbolic function, which maps values into $[-1,1]$.

Lastly, the output function, using the result from the previous step, calculates the value that will be transferred to the next nodes: Here, an identity function is often used (Kriesel 2007, 33-38).

Depending on the direction of the data propagation ANNs can be divided into feed-forward and recurrent networks. While in the feed-forward ANNs the calculated outputs propagate only forward, from the input layer to the output layer, the recurrent 
networks are bi-directional: the calculations are also routed back to the previous layer (Rybchynski, 2005).

The training of an ANN is managed by the training algorithm: A backpropagation algorithm is frequently used for the training. With this algorithm, the signals in the network are sent forward and the errors, differences between predicted and actual outcomes, propagate backwards (Duda et al. 2012, 288-296). During the learning process, weights of an ANN are adjusted to produce the desired output from the network. This back-propagation cycle is repeated until the desired criterion is reached: Common choices are either a minimum Mean Squared Error (MSE) over all trained cases, or a maximum number of epochs (number of times the training sent went through the network) (Kriesel 2007, 33-38).

There are two different ways of training an ANN using the back-propagation algorithm: The first method is the incremental training, where the weights of the connections are adjusted after each training case has been presented to the ANN (also known as the online training) and the second one is the batch training, where the weights in the ANN are only adjusted after the ANN has been presented with the entire training set (offline training) (Kriesel 2007b, 53-54).

\subsubsection{ANN software and libraries}

\section{$\underline{\text { MIRG ANN }}$}

Our research group has been using ANNs for predicting various outcomes such as mortality, length of stay, and other medical complications for different medical environments including the NICU. An ANN Research Framework (MIRG ANN RFW) 
was developed by previous students, using MATLAB's Neural Network Toolbox as a base, for implementing multilayer feed-forward ANNs (Ennett et al., 2004). The MIRG ANN RFW was automated to modify the number of hidden nodes from zero to $2 \mathrm{n}+1$, where $\mathrm{n}$ was the number of the input nodes, in the single-layer or multi-layer networks. An automated parameter update, for several driving parameters of an ANN, was implemented as well (Rybchynski, 2005). The values of the parameters were modified independently: One parameter was adjusted until the best parameter value (best result for a given stopping criteria) was reached, while the other parameters were kept constant. The best value for each parameter was carried forward to the next step (Rybchynski, 2005). To train the network, a logarithmic sensitivity index (Equation 2-2) was used: The training process was interrupted when there was no improvement in the index in subsequent 500 epochs (Ennett et al., 2002).

$$
\text { index }=- \text { Sensitivity }^{n} \cdot \log (1-\text { Sensitivity } \cdot \text { Specificity }) \text { [Equation 2-2] }
$$

After completing the training, the result files were processed to determine the best ANN model. Next, the weights of the selected model were manually extracted and passed to the prediction tool implemented in MATLAB. To make risk estimations for patients, data need to be passed to the tool manually for each patient (Rybchynski, 2005).

Despite having various features and functionalities, the manual steps involved in the risk estimation by the MIRG ANN RFW pose difficulties for the integration of ANN models into the real-time PPADS system. Also, the RFW and the prediction tool are based on MATLAB, which requires a commercial license to use. 


\section{$\underline{\text { Fast Artificial Neural Network library }}$}

In order to create ANN models, and make predictions in real time environment, we have explored several open sources libraries. We found the Fast Artificial Neural Network (FANN) library to be suitable to our work for the following reasons: firstly, the library implements feed-forward networks, which our research group has identified to be well performing machine learning methods for our medical datasets; secondly, the fast performance is one of the main features of the library, which is important in processing and analyzing real-time data; lastly, the library is implemented in $\mathrm{C}$ language which makes the FANN library based applications compatible with many software environments and portable to many different computer architectures or platforms (Nissen, 2007), (FANN, 2014).

FANN implements multilayer feed-forward ANNs with a support for both, fully connected and sparsely connected networks (FANN, 2014). One of the important advantages of this library is the implementation of several training algorithms and activation functions which, along with other network parameters, can be adjusted by users to achieve optimal performance.

Fifteen parameters, considered for the development of the software based on the FANN library, are listed here (FANN, 2014).

1. Connection rate.

2. Number of hidden layers.

3. Number of hidden nodes: Defines the number of nodes in each hidden layer. 
4. Connection weights: There are two options available in the library: Randomly assigning values to the weights within a chosen range (usually $[-1,1]$ ), or initializing the weights using Widrow-Nguyen algorithm. The latter is designed for speeding up the training process and requires the largest and smallest values of the input attributes (Nguyen and Widrow, 1990).

5. Activation functions: There are six activation functions that give an output between -1 to 1; Sigmoid symmetric, Gaussian symmetric, Elliot symmetric, Linear piece symmetric, Sine symmetric, Cosine symmetric.

6. Activation steepness: Determines the speed at which the activation function goes from the minimum to the maximum.

7. Training algorithms: There are four training algorithms available in the library; Incremental training, Batch training, Quickprop training (an advanced batch training), Rprop training (an advanced batch training).

8. Learning rate: Determines the speed at which the network attains a minimum in the criterion function.

9. Training error function: Two options are available; A standard linear function and a hyperbolic tangent error function.

10. Incremental training momentum: This parameter speeds up the training by adding a proportion of the previous weight-change value to the new value. 
11. Quickprop training weight decay factor: Determines how much the weights need to penalized to make sure that these do not become too high during the training.

12. Quickprop training maximum growth factor: Restricts the size of weights' growth.

13. Rprop initial step-size: Determines the initial step-size for weights.

14. Rprop step-increase: Determines how much the step size can increase during the training.

15. Rprop step-decrease: Determines how much the step size can decrease during the training.

\subsubsection{Performance measures}

A confusion matrix summarizes and visualizes the information about the classifier performance, showing the actual and the predicted classifications (Kohavari and Provost, 1998). Correctly made decisions are represented by the numbers along the major diagonal, whereas the numbers off this diagonal represent the errors (the confusion) between the various classes (Fawcett, 2006). In this work positive outcome is the less frequent outcome (i.e. mortality), thus a True Positive (TP) is a non-survivor correctly classified as a non-survivor and True Negative is a survivor correctly classified a survivor. Figure 2-3 illustrates a confusion matrix. 


\begin{tabular}{|c|c|c|}
\cline { 2 - 3 } \multicolumn{1}{c|}{} & $\begin{array}{c}\text { Actual outcome } \\
\text { positive }\end{array}$ & $\begin{array}{c}\text { Actual outcome } \\
\text { negative }\end{array}$ \\
\hline $\begin{array}{c}\text { Predicted outcome } \\
\text { positive }\end{array}$ & True Positive (TP) & False Positive (FP) \\
\hline Predicted outcome & False Negative (FN) & True Negative (TN) \\
\hline negative & & \\
\hline
\end{tabular}

Figure 2-3: Confusion matrix

By using the elements of the confusion matrix, the following performance measures can be calculated.

- Sensitivity, also called a True Positive Rate (TPR) or a Recall, is the rate at which non-survivors are correctly classified.

$$
\text { Sensitivity }=\frac{\mathrm{TP}}{\mathrm{TP}+\mathrm{FN}} \quad[\text { Equation 2-3] }
$$

- Specificity, also called a True Negative Rate (TNR), is the rate at which survivors are correctly classified.

$$
\text { Specificity }=\frac{\mathrm{TN}}{\mathrm{FP}+\mathrm{TN}} \quad[\text { Equation 2-4] }
$$

- The Positive Predictive Value (PPV) is the proportion of positive risk estimations that are correctly predicted. It is also referred to as Precision.

$$
\mathrm{PPV}=\frac{\mathrm{TP}}{\mathrm{TP}+\mathrm{FP}} \quad[\text { Equation 2-5] }
$$

- The Negative Predictive Value (NPV) is the proportion of negative risk estimations that are correctly predicted. 


$$
\mathrm{NPV}=\frac{\mathrm{TN}}{\mathrm{TN}+\mathrm{FN}}[\text { Equation 2-5] }
$$

- Accuracy (ACC) is the total number of cases that were predicted correctly.

$$
\mathrm{ACC}=\frac{\mathrm{TP}+\mathrm{TN}}{\mathrm{TP}+\mathrm{FN}+\mathrm{FP}+\mathrm{FN}}[\text { Equation 2-6] }
$$

- F1-score uses both the precision and recall of the test, acting as a weighted average of both these measures.

$$
F 1=\frac{2 T P}{2 T P+F P+F N} \quad[\text { Equation 2-7] }
$$

- The Mathews Correlation Coefficient (MCC) is a correlation coefficient between the observed and predicted classifications.

$$
\mathrm{MCC}=\frac{\mathrm{TP} \cdot \mathrm{TN}-\mathrm{FP} \cdot \mathrm{FN}}{\sqrt{(\mathrm{TP}+\mathrm{FP})(\mathrm{TP}+\mathrm{FN})(\mathrm{TN}+\mathrm{FP})(\mathrm{TN}+\mathrm{FN})}}[\text { Equation 2-8] }
$$

The performance measures calculated by the equations $2-3$ to 2-7 take values between 0 and 1 , with 1 being the ideal value. The MCC, calculated through the equation $2-8$, returns a value between -1 and 1 , where 1 is the perfect risk estimation.

Another accuracy measure that is being used to measure and compare classifier performances is the Receiver Operating Characteristic (ROC) curve. It is a graph of the false positive rate (1-specificity) versus the true positive rate (sensitivity). Each point on the ROC curve represents a sensitivity/specificity pair corresponding to a particular decision threshold. A test with perfect discrimination (no overlap in the two distributions) has a ROC curve that passes through the upper left corner (100\% sensitivity, $100 \%$ specificity). Therefore the closer the ROC curve is to the upper left corner, the higher the overall accuracy of the test (Zweig and Campbell, 1993), (MedCalc, 2014). Figure 2-4 illustrates an ROC curve. 


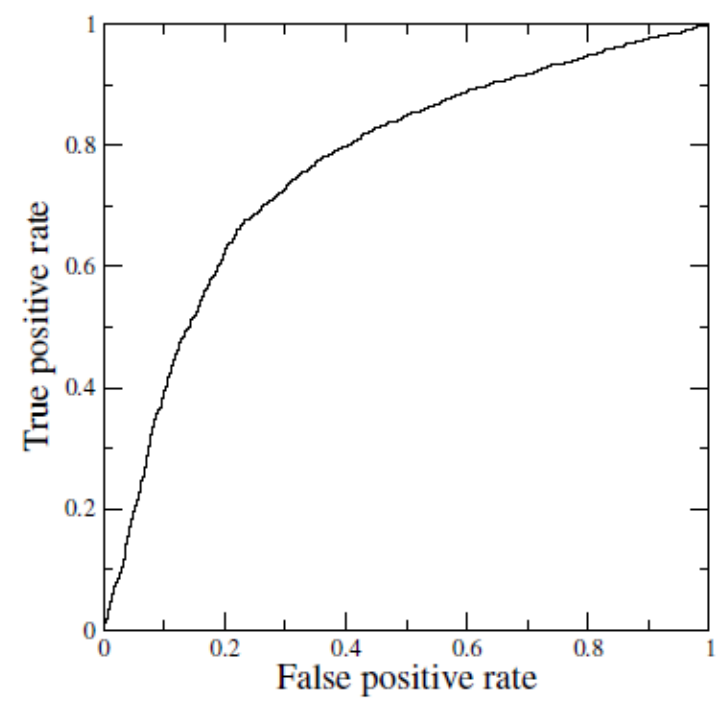

Figure 2-4: An ROC curve (Fawcett, 2006)

In order to compare the performance of the classifiers the Area Under ROC Curve (AUC) is often used. AUC takes values between 0 and 1, and since random guessing will produce an AUC of 0.5 , a realistic classifier should achieve a value higher than that. The AUC value can be interpreted as the probability for a classifier to rank a randomly chosen positive case (i.e. death or presence of a disease) higher than a randomly chosen negative case (Fawcett, 2006). 


\section{Chapter: Methodology}

\subsection{Neonatal Mortality Prediction}

This section describes the methodology used to develop Artificial Neural Network (ANN) models, and the integration of these models with the Physician-PArent Decision Support (PPADS) system.

\subsubsection{Datasets and data preparation}

The datasets used for the development of neonatal risk estimation models consisted of summation data from the years 2010 and 2011, retrieved from the Clinical Data Repository (CDR) in the Neonatal Intensive Care Unit (NICU) at the Children's Hospital of Eastern Ontario (CHEO).

The first dataset, containing 506 patient cases and having a 6\% mortality rate, was summary data from admission up to the first 12 hours after admission. The second dataset, with 630 cases and a 5\% mortality rate, was summary data from admission up to the 48 hours after admission. The patient cases contained in the datasets were deidentified by the CDR system in order to protect patient privacy: To each patient case an arbitrary study ID was automatically assigned by the system. The attributes, 18 in number, were representing patients' vital signs collected every minute and common blood based laboratory tests that are performed non-invasively. To eliminate the inclusion of outliers due to monitoring noise or artifact, the median of the top or bottom five values recorded for vital signs for the highest or lowest attribute measurements respectively were used; For heart and respiratory rate measurements our clinical partners advised to 
exclude values that are above/below certain level (less than 30 and more than 340 beats per minute, greater than 130 respirations per minute) (Gilchrist, 2012). The attributes are summarized in Table 3-1.

Additionally, a dataset consisting of 3 attributes (lowest serum $\mathrm{pH}$, lowest blood pressure, and lowest heart rate) of 48 hour summation data was used for creating prediction models. These attributes were found to be the most important in estimating the risk of mortality during the first 48 hours of the NICU admission in the previous work of our group (Gilchrist, 2012).

In order to compare the performance of the MIRG ANN Builder (using the FANN library as a base) to the MIRG ANN RFW, the datasets used in this work were the same as in the previous work by J. Gilchrist. The missing values in the dataset were imputed using the CBR system with k-NN and the normalization was performed through the modified Z-score transformation formula (Gilchrist, 2012).

Table 3-1: List of attributes

\begin{tabular}{|cc|}
\hline \hline & Attributes \\
\hline Lowest mean blood pressure & Highest mean blood pressure \\
\hline Lowest heart rate & Highest heart rate \\
\hline Lowest serum glucose & Highest serum glucose \\
\hline Lowest serum sodium & Highest serum sodium \\
\hline Lowest pO2 level & Highest pO2 level \\
\hline Lowest respiratory rate & Highest respiratory rate \\
\hline Lowest blood hematocrit & Highest blood hematocrit \\
\hline Lowest absolute neutrophil count & Highest absolute neutrophil count \\
\hline Lowest platelet count & Highest platelet count \\
\hline
\end{tabular}




\subsubsection{Cross validation method}

The 5-by-2 cross validation method was used to obtain more reliable risk estimation models. The methodology used in the previous work, where train and test sets were created to perform the 5-by-2 validation of decision trees, was adapted to achieve a division into train, test and verification sets for ANNs. The process was as follows:

1. Data were separated into mortality and non-mortality sets.

2. Both, the mortality, and non-mortality sets were randomized and split into two equal sized sets (A and $\mathrm{B})$

3. The set A was split into A1 and A2 for both, the mortality and non-mortality sets, leaving the set B as a whole set. Then the process was reversed: The set B was split into B1 and B2, and the set A was left as a whole set.

4. The mortality and non-mortality sets B, A1, A2 were merged to form the train, test and verification sets respectively, and were labeled as Set \#1. Then, the sets A, B1, B2 were merged to form the second set, Set \#2.

To create ten different train, test and verifications sets (Set \#1 through Set \#10), the steps 2 through 4 were repeated five times in total.

The division of the mortality and non-mortality sets into equal parts ensures the equal number of positive and negative cases to train, test, and verify the ANN models. The 5-by-2 cross validation method is advantageous since it allows all patient cases to be used during the training, not letting any case that leads to better predictions to be excluded (Dietterich, 1998), (Gilchrist, 2012). The 5-by-2 method is summarized in Figure 3-1. 


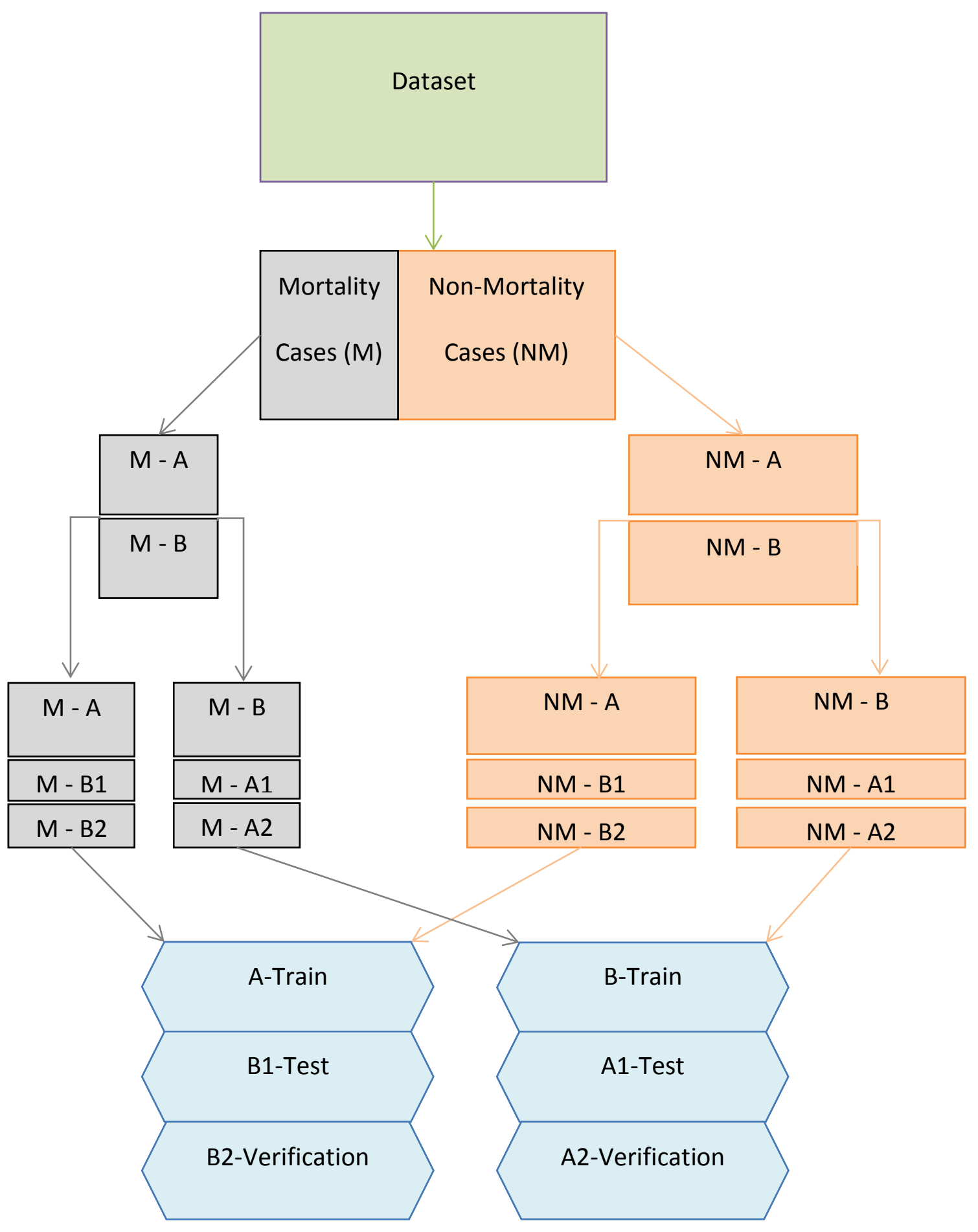

Figure 3-1: 5-by-2 Cross Validation Method 


\subsubsection{Formatting the data}

In the FANN library, the input files must follow certain formatting guidelines: The first line in the input file must include the number of cases, the number of attributes and the number of outputs. This should be followed by a line of inputs and a line of outputs for each case. The formatting requirements are summed up in Table 3-2.

Table 3-2: Input File Format for the FANN library

\begin{tabular}{|c|c|c|}
\hline \multicolumn{3}{|c|}{ FANN Format } \\
\hline Number of cases & Number of input attributes & Number of outcomes \\
\hline \multicolumn{3}{|c|}{ Case $i$ inputs } \\
\hline \multicolumn{3}{|c|}{ Case $i$ outputs } \\
\hline
\end{tabular}

$\mathrm{i}=1, \ldots, \mathrm{n}$; where $n$ is the number of cases

\subsubsection{Developing ANN models}

The FANN library implements feed-forward ANNs and allows users to create, train, and test networks with various options for the network architecture: activation, training and the parameters that drive the learning of the ANN. The options and the parameters are set to their default values, and the users of the library are expected to adjust these to achieve an optimal ANN model performance. In order to automate the process of the option selection and the adjustment of parameter values when creating ANN models, automated software was developed and utilized. The software, called 
MIRG ANN Builder, also included a function to run an existing network to calculate the prediction scores.

The operation of the MIRG ANN Builder is as follows.

\section{Set up of the input options}

The command line tool is available in either of two ways: in an automatic form, the input options already selected, or in a generic form, giving a user the possibility to perform the selection. Following input options are available:

1. The running mode: "fast", "medium", "slow", or "scores".

2. Choosing the number of the hidden layers in the network.

3. 5-by-2 cross validation: on or off.

4. Sub-parameters adjustment: on or off.

5. Choosing the thresholds for selecting the networks.

\section{Running}

Case 1: "fast", "medium", and "slow" mode is selected

In order to allow users to choose between a quick or a detailed scanning of the options and the parameter values, the program is available in three different operational modes: The "fast" mode tests a few combinations of options and parameter values (approx. $0.5 \%$ of all possible combinations, excluding the number of hidden nodes, which depends on data), the "medium" mode tests more options but 
not all (approx. 7\%), and the "slow" mode runs through all possible combinations of options and parameter values (approx. $96 \cdot 10^{3}$ combinations).

After the input options are selected, a directory to save the results is created to organize the networks which also stores and creates the following files: a result file, intended for recording the options and parameters trials with their outcomes, and an information file, containing the selected input options and the starting time of the program.

The program takes a set of options and values, and then a network is created and trained until the desired MSE value (0.001, the default error value of the FANN library) is achieved. Next, the trained network is tested using the test set. If the sensitivity and the specificity values pass the selected test threshold, then the network is verified using the verification set; otherwise, the program goes back to the step of setting the options and values. Consequently, if the network passes the selected verification threshold when tested on the verification set, then the network weights and the options are saved into a network file and the result's file; otherwise, it goes back to the step of setting the options and values. After accomplishing previous steps, the program takes another set of options and parameters and repeats the process. This cycle is repeated until the upper range of possible values is reached. At the end of the program, the end time and the elapsed time are written into the information file. The ANN options and parameters with their ranges are summed up in Table 3-3. 


\section{Case 2: "scores" mode is selected}

When the "scores" mode is selected, the program reads an already existing network file and a dataset with/without known outcomes, and outputs the prediction scores into a Comma Separated Value (CSV) format file (.csv extension).

Figure 3-2 shows the operation of the MIRG ANN Builder. 
Table 3-3: List of the options and parameters tested by the MIRG ANN Builder

\begin{tabular}{|c|c|c|c|c|}
\hline \multirow[b]{2}{*}{ ANN Options and Parameters } & \multirow[b]{2}{*}{ Ranges } & \multicolumn{3}{|c|}{ Increments } \\
\hline & & Fast Mode & $\begin{array}{c}\text { Medium } \\
\text { Mode }\end{array}$ & $\begin{array}{l}\text { Slow } \\
\text { Mode }\end{array}$ \\
\hline Hidden layers $^{1}$ & {$[1,3]$} & $\mathrm{x}$ & $\mathrm{x}$ & $\mathrm{x}$ \\
\hline Connection rate & {$[0.1,1]$} & 0.4 & 0.3 & 0.1 \\
\hline Number of hidden nodes ${ }^{2}$ & {$[1,2 \mathrm{n}+1]$} & 30 & 10 & 1 \\
\hline Weights & {$[1,2]$} & 1 & 1 & 1 \\
\hline Activation function & {$[1,6]$} & 3 & 1 & 1 \\
\hline Activation steepness & {$[0.1,1]$} & 0.4 & 0.3 & 0.1 \\
\hline Training Algorithm & {$[1,4]$} & 3 & 1 & 1 \\
\hline Learning rate & {$[0.1,1]$} & 0.4 & 0.3 & 0.1 \\
\hline Training Error Function & {$[1,2]$} & 1 & 1 & 1 \\
\hline Incremental training momentum $^{3}$ & {$[0,1]$} & 0.5 & 0.2 & 0.1 \\
\hline Quickprop training decay factor ${ }^{3}$ & $\begin{array}{l}{[-0.0001,-} \\
0.001]\end{array}$ & -0.0005 & -0.0002 & -0.0001 \\
\hline $\begin{array}{l}\text { Quickprop training maximum } \\
\text { growth factor }\end{array}$ & {$[1,2]$} & 0.5 & 0.2 & 0.1 \\
\hline Rprop training initial step ${ }^{3}$ & {$[0.1,1]$} & 0.5 & 0.2 & 0.1 \\
\hline Rprop training step increase factor ${ }^{3}$ & {$[1,2]$} & 0.5 & 0.2 & 0.1 \\
\hline $\begin{array}{l}\text { Rprop training step decrease } \\
\qquad \text { factor }^{3}\end{array}$ & {$[0.1,1]$} & 0.5 & 0.2 & 0.1 \\
\hline \multicolumn{5}{|c|}{$\begin{array}{l}{ }^{1} \text { this structural parameter is not included in the automated checking and is selected when } \\
\text { starting the program }\end{array}$} \\
\hline
\end{tabular}




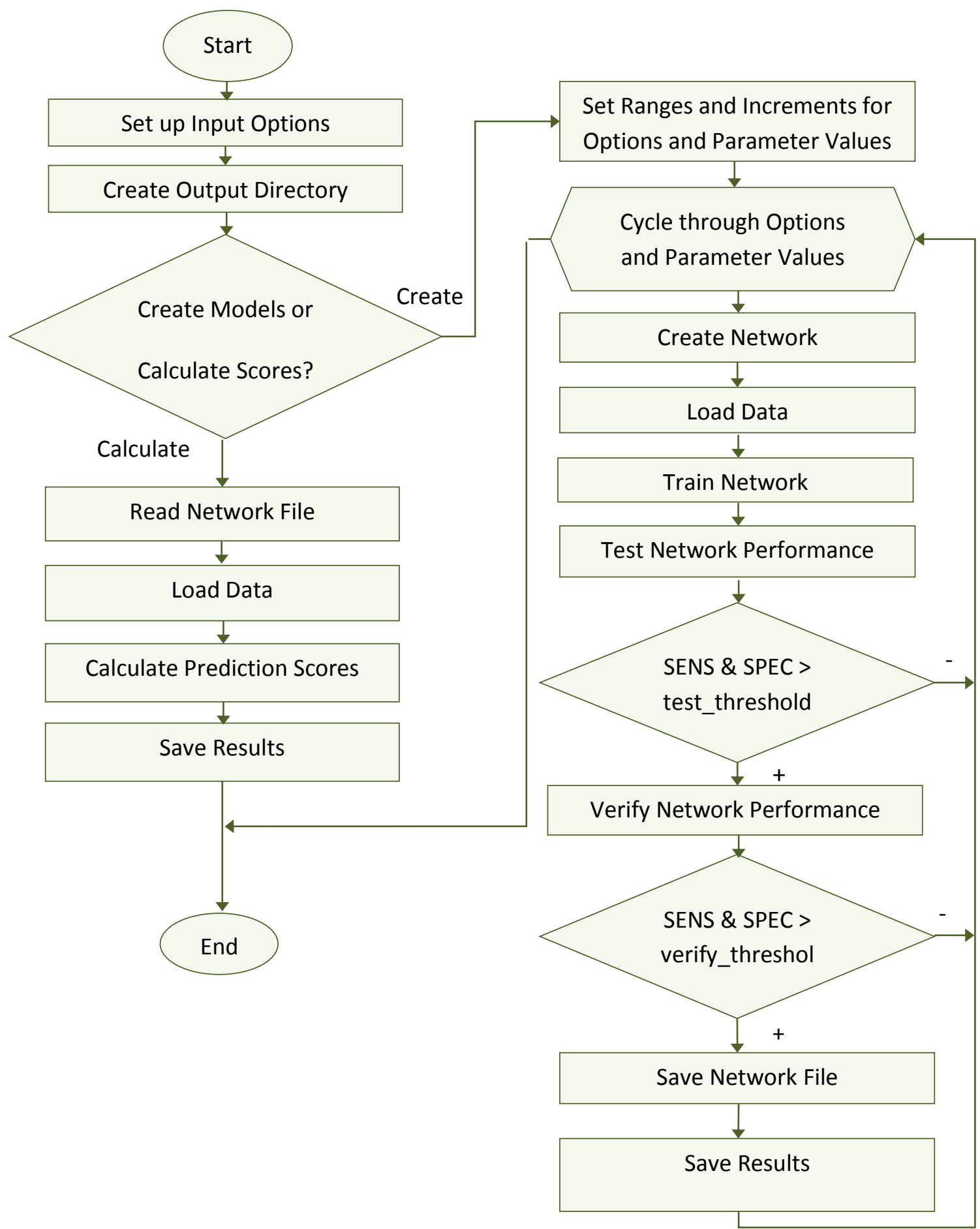

Figure 3-2: Flow Chart: MIRG ANN Builder.

Here, SENS is the Sensitivity, SPEC is the Specificity, and test_threshold and verify_threshold are selected at the program's start. 


\subsubsection{Selecting the ANN models}

The MIRG ANN Builder was used to create ANN models, for all three datasets, with fast and medium modes with or without the sub-parameters option activated.

Networks with one hidden layer were tested. The selection thresholds, which decide whether the networks would pass to the verification stage (test threshold) and later be saved in a network file (verification threshold), were set low for fast modes: the test threshold was $10 \%$ (for both the sensitivity and the specificity) and the verification threshold was $20 \%$. These values were increased for medium modes; to $30 \%$ and $40 \%$ respectively. This restriction was intended for saving computational time when writing the files and reducing the size of result files, thus making the selection process faster and easier.

With 5-by-2 cross validation, ten folders were created containing a result file and the network files. The result files record the options and the values of the parameters with which the ANNs were created, along with the performance results on the test and verification sets. All result files were sorted, to find the networks with the highest sensitivity results. Afterwards, further performance measures were calculated for the selected networks.

The sorting and the calculation of the performance measures of ANN models were accomplished by following steps.

1. All networks with the highest sensitivity/specificity on the verification sets were selected.

2. From the selected networks, one network with the highest sensitivity/specificity result on the test set was chosen as the best performing one. This step was 
performed to extend the confidence in the results; if the models give similar and good results on two different datasets, then the models are more likely to generalize well, that is perform an accurate classification of new and unseen cases.

3. Step 1 and Step 2 were repeated for all 10 result files.

4. MIRG ANN Builder was employed again, in the "scores" mode, to create .csv files containing the predictions and the actual outcomes.

5. The files from the previous step were used to calculate further performance measures: PPV, NPV, ACC, MCC, F1-score and AUC. All results were directed to a single .csv file, which, upon the completion of this step, contained 10 rows. Each row in the file represented the performance of one network that was trained, tested and verified using one of the 5-by-2 cross validation sets (Set \#1 through Set \#10).

6. The average and standard deviation of the rows were calculated to obtain the final result, which represents the performance of ten networks in a single set of results.

\subsubsection{Presenting the risk}

The output that an ANN is providing is a relative score between -1 and 1. This score is applied to classify the cases as positive ("death") or negative ("survival"): if the score is greater than or is equal to zero (a threshold used in this work), then the case is classified as positive, otherwise it is classified as negative. When presenting the predictions through the PPADS tool, our clinical partners specified a preference of seeing the predictions in risk categories (Gilchrist, 2012). Risk categories were: Low $(-1 \leq$ score $<-0.5)$, medium $(-0.5 \leq$ score $\leq 0.5)$, and high $(0.5<$ score $\leq 1)$. 


\subsubsection{Integrating the ANN models into the PPADS}

This section describes the design and the development of the tool that integrates the ANN models with the PPADS system. The Connection Tool should retrieve the medical data of active patients from CDR, utilize ANN models to calculate their risk assessments, and pass this information to the PPADS for a display to physicians and to parents as decided by the physicians and the parents. Figure 3-3 illustrates how the CDR, the PPADS system, the MIRG ANN Builder and the Connection Tool should interact.

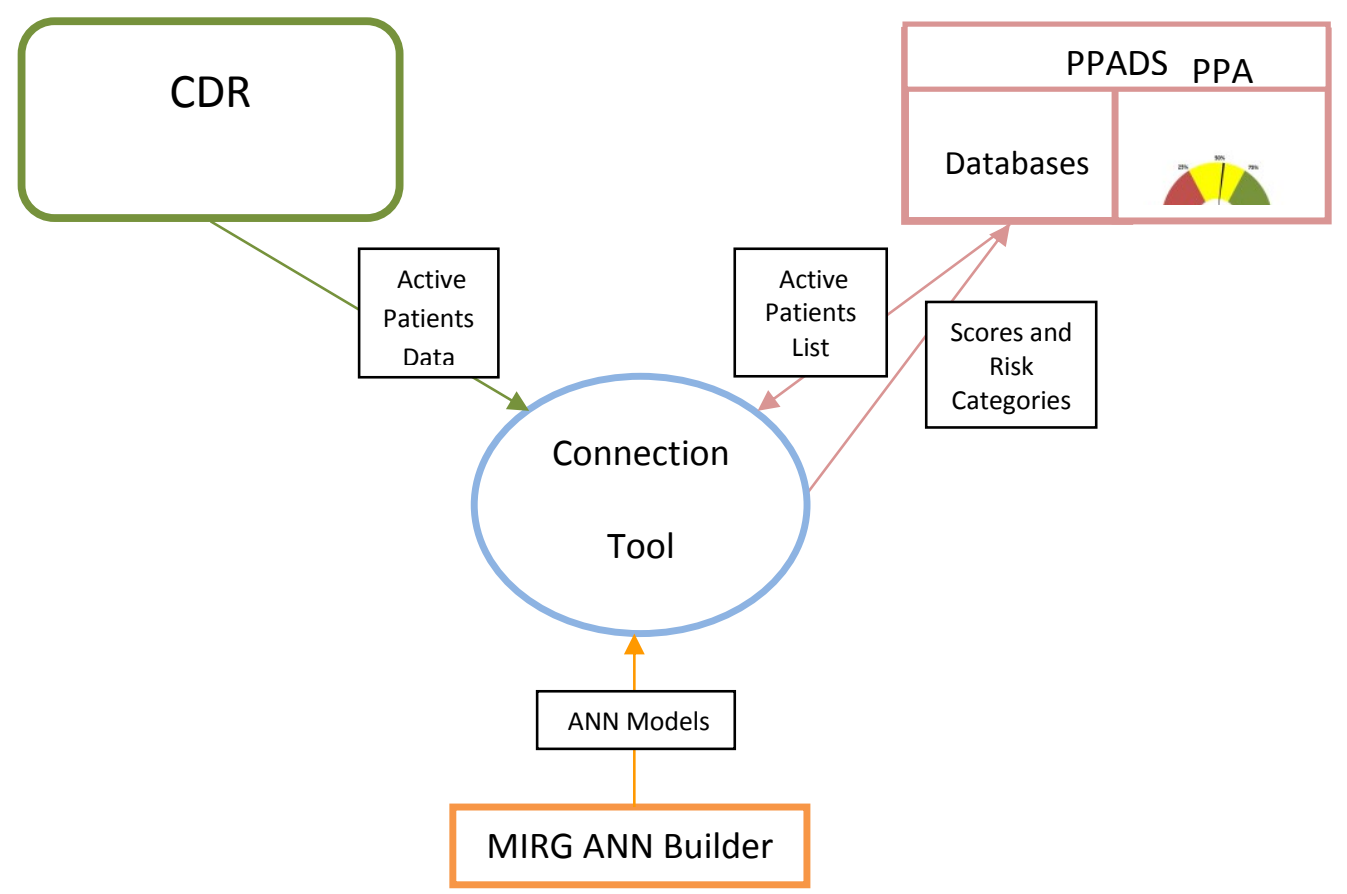

Figure 3-3: Integration of ANN models with PPADS 
- Active Patient List: Should contain the study IDs and patient IDs (Drupal ID) of patients who have a PPADS account and have not been yet been discharged from the NICU.

- Active Patient Data: Should contain the medical data (18 attributes) extracted for the patients who are on the Active Patient List.

- ANN models: The Connection Tool should apply the ANN models to calculate the prediction scores, and convert these into risk categories.

- Scores and Risk Categories: The information on scores and risk categories should be passed to the databases of the PPADS system for a display in the interfaces.

For ANN models to make a prediction about a single patient case, the program needs to access patients' medical data from the real-time CDR. The latter is only accessible from within the hospital's secure network to ensure patient data safety. In order to create an environment for the development of the tool, the summation data from CDR (excluding the information about the outcomes) was imported to a MySQL database on a local machine in our research lab at Carleton University.

The Connection Tool was developed in $\mathrm{C}++$ using MySQL Connector library. It operates as follows.

\section{Set up of the input options}


Similar to the operation of the MIRG ANN Builder, the command line tool for the integration is available in either of two ways: in an automatic form, the input options already selected, or in a generic form, giving a user possibility to perform the selection. Following input options are available:

- Server connection details: host, user, password for the CDR and the PPADS.

- The names of databases and tables to connect to.

- The names and the locations of the ANN models.

- The type of the outcome to be predicted (mortality or other medical complications).

\section{Running}

When the programs start to run, a connection to the CDR and to the databases of the PPADS system is established using the input details. The list of active patients is queried from the PPADS system to obtain the medical information about these patients from the CDR. The application operates case by case in order to not mix the medical information and the predictions for different patients. Next, the data are normalized and fed into the prediction model to obtain the risk scores. The tool also converts these scores into risk categories and writes both the scores and the categories into the databases of the PPADS system. Additionally, the information about the prediction model (the sensitivity, the specificity, and the AUC values) is also retained in order to display in the clinician's interface of the PPADS system. 
Here, it is assumed that the patient information does not contain missing values, since the CBR system used for the imputation of missing values was not designed for a realtime operation. A new system for replacing missing values with real-time data streaming is a part of the future work. A flow chart of the operation of the Connection Tool is shown in Figure 3-4.

To present the operation of the Connection Tool, 20 patient accounts have been created in the PPADS tool. The tool was utilized to calculate the predictions of mortality for these patients by querying their data from the CDR, and to pass the information to the databases of the PPADS tool. 


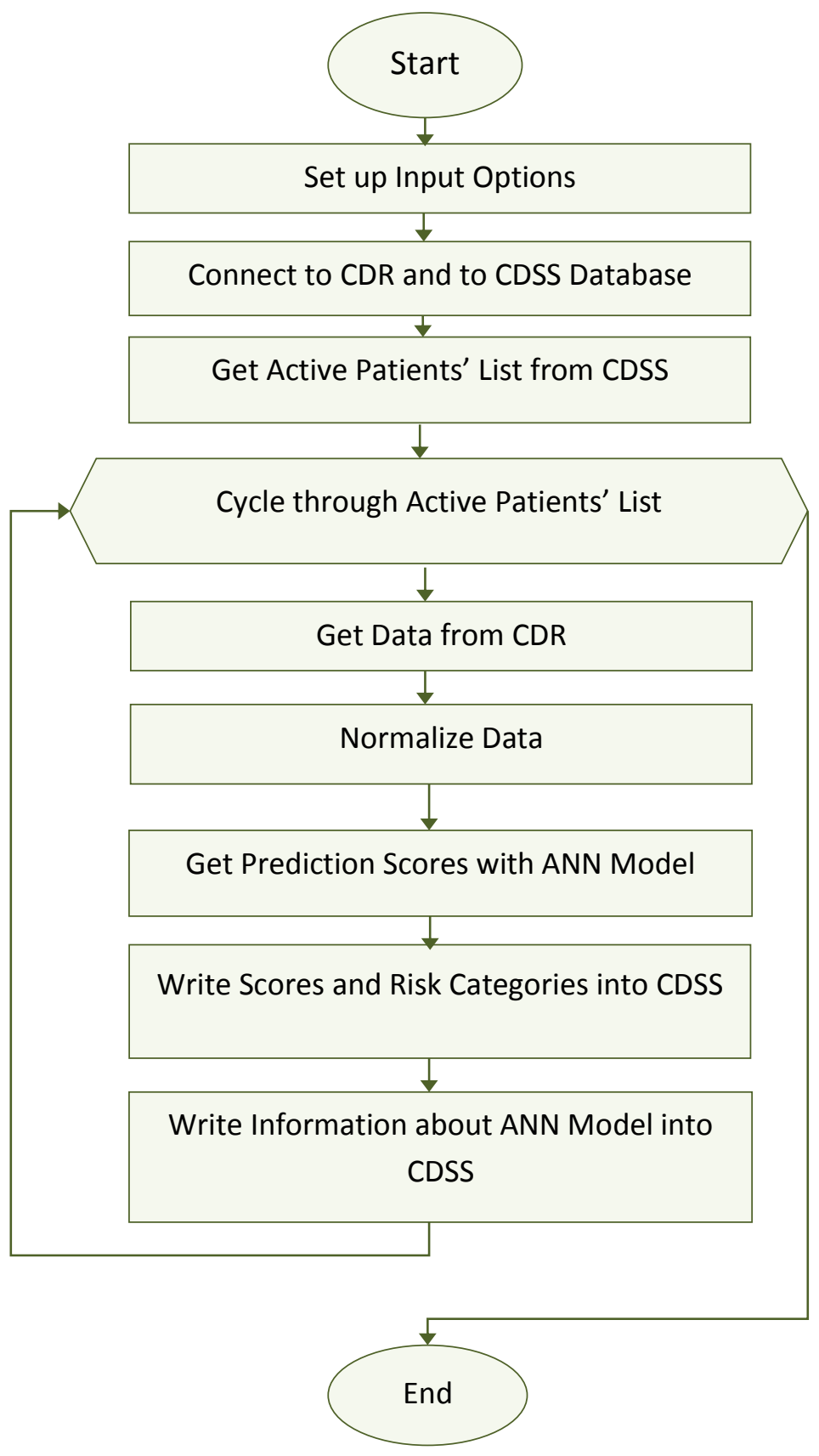

Figure 3-4: Flow Chart: Connection Tool

The CDSS is one of the databases of the PPADS system. 


\subsubsection{Automation of the steps}

The data preparation (normalization, division into 10 sets, formatting), and the selection of the ANN models (sorting of the results, calculating performance measures) were automated using Borne-Again-Shell (BASH) scripts.

\section{Data normalization tool:}

The normalization of data is accomplished in two steps: first, obtaining normalization parameters (mean, standard deviation) from data in the database; second, using these parameters to normalize the data with Z-score formula (Equation 2-1).

To develop the automated tools, the data imported into a MySQL database during the integration of the models with the PPADS tool was utilized.

I. A tool was created in $\mathrm{C}++$ that obtained the normalization parameters from data: Using SQL queries, it found the means and the standard deviations of each 18 attributes in data and wrote these values to both the CDSS database and to a file named normalization.csv.

II. A BASH script was written to perform the normalization on mortality.csv and nonmortality.csv files; these files contained the mortality and non-mortality patient cases. First, it loaded the normalization.csv and wrote the means and the standard deviations of each feature into a temporary file, then the contents of the mortality.csv/nonmortality.csv were added to the temporary file (the columns which represented the study IDs and the outcomes were excluded). Columns in the temporary file were processed one by one: the first row contained the mean value and the second row the standard deviation for that column; using these 
statistical measures, the rest of the values in the column were normalized with the Z-score transformation formula. Normalized cases were combined into a final .csv file with study IDs and outcomes.

\section{$\underline{\text { Data Division Tool }}$}

In order to divide the data into 10 sets for the 5-by-2 cross validation, the create_5by2_folds.sh script written by J. Gilchrist (Gilchrist, 2012) was adapted to divide data into train, test and verification sets. The script was written to accept as input parameters the mortality.csv and non-mortality.csv files. It first randomized the data, then divided it into two parts: the first part became the training set, while the second part was divided into two to become the testing and the verification sets; the mortality and nonmortality cases were merged for each of these sets to form the Set \#1 (train_fold_1_a.csv, test_fold_1_a.csv and verification_fold_1_a.csv). Then the process was reversed, so that the second set of the randomized division became the train set, and the first one was divided into two parts to become the test and the verification sets; again, the mortality and non-mortality cases were combined to form the Set \#2 (train_fold_1_b.csv, test_fold_1_b.csv and verification_fold_1_b.csv). The process was repeated four more times to create 10 different train, test and verification sets; 30 files in total were created.

\section{Formatting Tool:}

The formatting script took each of the files in the previous step and performed the following: Number of rows (patient cases), number of columns (attributes) and number of outputs (known outcomes) were calculated from files and written on the first 
line of the data file. Rest of the file consisted of single line of attributes and single line of outputs for each patient case.

\section{ANN Model Selection Tool:}

In order to simplify and automate the process of network selection from result files, a sorting script was written. The script selected the networks either by sensitivity or specificity. For a selection according to sensitivity, the tool operated as follows: for each result file (10 sets after 5 -by-2 cross validation), it sorted the rows according to sensitivity values on the verification set, and then directed the results into another .csv file called sens_first_sort_.csv. Next, the rows containing the maximum sensitivity were stored into a temporary file. The temporary file was sorted three more times: first, according to verification specificity, then test sensitivity, and lastly test specificity. The sorted results were directed into the sens_second_sort.csv. The network on the first row was selected as the best performing one. The string value in the fifth column of the first row, representing the path to the network file, was parsed to extract the name of the network file. Using the filename, the network was copied into a separate folder. After all 10 result files were sorted; the folder contained 10 selected networks.

A selection according to the specificity worked in a similar way. 


\section{Performance Measures Calculation Tool:}

For the purpose of calculating further performance measures of selected networks, the MIRG ANN Builder can be used in the "scores" mode to calculate the prediction scores and output these, along with the actual outcomes, into a scores.csv file.

The script written to calculate the performance measures used the scores.csv file. First, it found the number of positive and negative cases. Then, it calculated the elements of the confusion matrix (Figure 2-3). As next, the Positive Predictive Value (PPV), Negative Predictive Value (NPV), Accuracy (ACC), Matthews Correlation Coefficient (MCC) and F1-Score were calculated. The values were directed into a temporary file. Afterwards, the ROC points and the Area Under Curve (AUC) were calculated. The AUC values were added to the temporary file containing the values calculated previously. These steps were repeated for each of the ten result files, and all results were saved into a classifier_statistics.csv file. As a last step, the means and standard were calculated and directed to the classifier_statistics_final.csv file.

\section{Combined Scripts}

To automate the data preparation entirely, a script was written that combined all preparation steps by calling the scripts for each of the steps: the normalization, the data division and the formatting.

Additionally, a combined script was written to perform the ANN model selection and calculation of accuracy measures automatically.

These scripts can be combined with a MIRG ANN Builder binary to perform data preparation, ANN model creation, and sorting of the results without any user supervision. 


\subsection{PPADS Pilot Study}

This section describes methods used in conducting the short pilot study to test the usability of the PPADS system where parents whose infant has not graduated (not survived) from the NICU at CHEO were recruited for this study.

The methodology largely followed the protocol of the previous usability study conducted by S. Weyand (Weyand, 2011). The purpose of the previous study was to get an initial feedback about the PPADS system from parents whose child survived in the NICU. In this study, parents who have experienced the loss of an infant in the NICU participated. The feedback they provided on usefulness and ease of use of the PPADS system is invaluable, since they went through the stress and difficult decision-making process for their critically ill infant, who eventually died in the NICU, and thus they would know the needs of parents in similar situations (Bariciak, 2014).

Ethics approval for the study, conducted at CHEO, was obtained from the CHEO Research Ethics Review Board and Carleton University Research Ethics Board. The ethics approval forms are included in the Appendix C.

\subsubsection{Aim of the study}

The study aimed to determine the following (Rubin and Chisnell 2008, 4-6), (Weyand, 2011):

- Usefulness: The degree that the tool helps the users, and the degree with which the users desire to use the system.

- Efficiency: How fast users can navigate through the tool with accuracy.

- Effectiveness: The ease with which the users can navigate through the system. 
- Acceptability: The comprehensibility and amount of information provided in the system.

- Satisfaction: Users' overall perceptions and opinions of the system.

\subsubsection{Recruitment}

The participants have been recruited using the following inclusion criteria:

- Participants had an infant who died in the NICU at least 12 months or more from the start date of the study.

- Participants should not currently have any infant(s) in the NICU.

- Participants must be 18 years of age or older (to be able to provide informed consent)

Since the study involved a vulnerable population, (the parents have had previously experienced the death of an infant in the NICU), the selection of potential participants included approaching those families who were either still in contact with the NICU Social Worker, and were coping well emotionally, and/or those families who had expressed interest in participating in research projects or bereavement initiatives in the NICU.

The participants were identified by Dr. Erika Bariciak and the NICU Social Worker, from discharge records kept in the NICU, and recruited by the research coordinator at CHEO (Samantha Somers) with the help of Dr. Erika Bariciak.

Dr. Thierry Lacaze, the Chief of the Department, sent each identified family a recruitment letter asking if parents would be interested in participating in the research study. Each parent would participate separately. If parents chose to participate, they were given the option to contact the NICU research coordinator by phone or by email to get 
more information, or had her contact them through information they had provided on the returned recruitment letter. After contacting the study coordinator, parents were provided with a copy of the information sheet and consent form describing the study in more detail, and were asked to make an appointment for the study session. The studies were conducted away from the NICU, in a conference room at the West Wing of CHEO. The participants received reimbursement for their parking fee at CHEO.

\subsubsection{Study session}

Participants were asked to go to the main lobby at CHEO, and were greeted and escorted to the conference room. The only people who were present throughout the study were the study assistant (Hasmik Martirosyan) and Dr. Erika Bariciak. The parents were free to withdraw from the study at any time and were offered an emotional support by the NICU Social Worker if needed.

The participants were read the first part of the participant speech. Next, the participants were provided with the information sheet and the consent form. Upon signing the consent form, parents were asked to complete the background questionnaire. The participants were provided with a laptop computer with the PPADS tool open and the task sheet. The participants read information about two hypothetical cases involving sick infants in the NICU. They were asked to use the decision support system to better understand the clinical status of the babies and what the various care options are.

Figures 3-5 to 3-9 illustrate the different components (modules) of the PPADS system that were viewed and evaluated by the participants of the pilot study. 


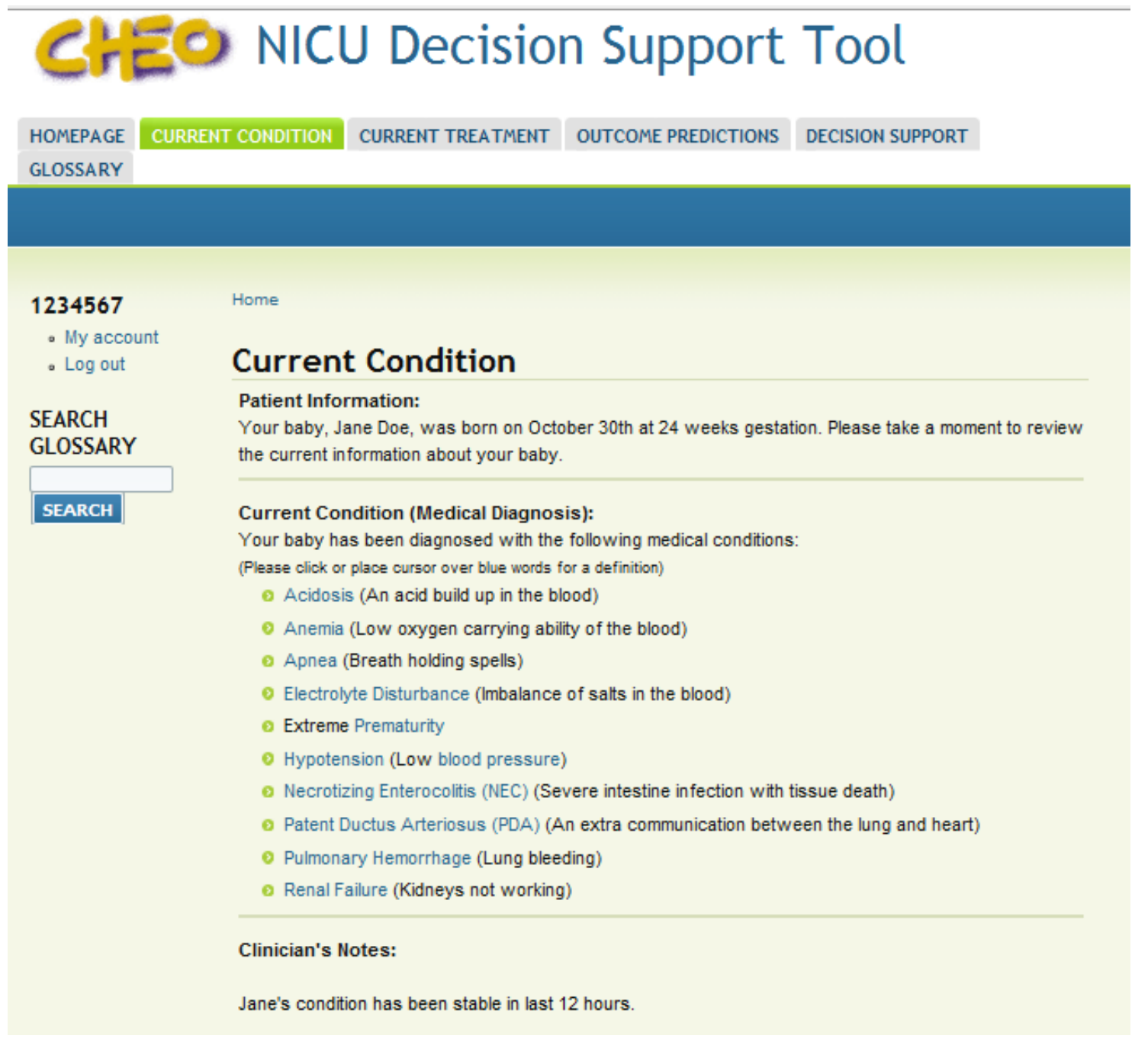

Figure 3-5: PPADS - Current Condition 


\section{Current Treatment}

Current direction of care:

The current direction of care is:

- Full, active care

\section{Current therapies:}

The current therapies being used are:

(Please click or place cursor over blue words for a definition)

(6) Antibiotics

( Arterial Catheters

- Artificial Nutrition (TPN)

(?) Artificial Ventilation

( Blood Pressure Medications

() Blood Transfusions

( Central Venous Lines

( High Frequency Oscillation Ventilation (HFOV)

( Intravenous Fluids

- Kidney Function Medications

- Nasogastric Tube (NG Tube)

Figure 3-7: PPADS - Current Treatment

\section{Outcome Predictions}

Prediction Models:

The prediction models used information from thousands of babies with similar conditions as your baby in reaching the following predictions.

Please note that these probabilities are only estimates and not a guarantee.

Predicted Risk of Mortality (Death):

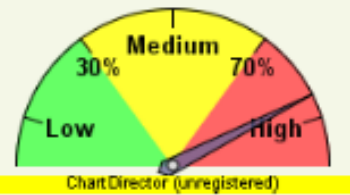

For 100 babies with similar problems as your baby: 85 will die.

Updated at 12:44, Nov 3rd, 2014.

\section{Predicted Risk of} Bronchopulmonary Dysplasia (BPD) (Chronic Lung Disease):

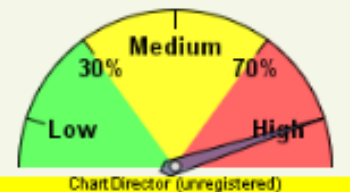

For 100 babies with similar problems as your baby: 90 will have $B P D$.

Updated at 12:44, Nov 3rd, 2014.
Predicted Risk of Severe Intraventricular Hemorrhage (IVH) (Brain Bleeding):

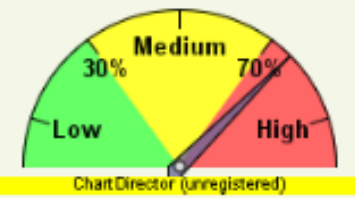

For 100 babies with similar problems as your baby: 75 will have severe IVH.

Updated at 12:44, Nov 3rd, 2014.

Figure 3-6: PPADS - Outcome Predictions 


\section{Decision Support}

You have been asked to consider a change in the direction of care.

Direction of care options:

You are being asked to consider the following directions of care:

- Full, active care

- Non-escalation of care

- Do not resuscitate order (DNR)

- Withdrawal of life support / palliative care

While you are considering a change in the direction of care, the current direction of care (Full, active care) will continue.

Reason change in direction of care has been suggested:

The reason a change in the direction of care has been suggested is:

- Death is most likely to occur despite full active care

- We believe your baby is suffering

- Your baby is likely to have severe developmental handicaps if he/she survives

\section{Change in direction of care decision support form:}

This decision support form is a questionnaire that will help you to:

- Clarify the different options

- Understand how your values and beliefs influence your decisions

When you are done and submit the form you can print it for future reference. You can also edit your results at any time. This form does not make the decision for you or come to any conclusions; it is just meant to help guide you in your decision making.

- Start a new decision support form

\section{Figure 3-8: PPADS - Decision Support}




\section{Decision Support}

Clarify the decision (Page 2 of 6)

When does this decision have to be made?:
Within Hours
Within Days
Within Weeks
I don't know
Other...

How far along are you with this decision?:

I have not thought about it yet

I am considering the options

I am close to choosing an option

I have already made a choice

\section{SAVE DRAFT < PREVIOUS PAGE NEXT PAGE >}

Figure 3-9: PPADS - Decision Support Form

Upon completion of the tasks, the participants were given the evaluation questionnaire. The completing of the tasks and questionnaires lasted approximately $30-45$ minutes. Upon completion of the study, participants were escorted to the hospital exit, and their parking passes were paid.

\subsubsection{Data collection}

Data were collected in two main ways: questionnaires and observations under controlled conditions. The participants were asked to complete two questionnaires: one before and one after using the PPADS system. Additionally, observations about what the participants said and did during their interaction with the tool were noted. The following information was recorded: 
- Number of tasks completed correctly with and without questions

- Qualitative data ( comments, quotes, suggestions for improvements)

Each participant was given a participant ID number (randomly generated by the Excel random number generator) which was located on every form/questionnaire. This was

done to ensure that the participant forms did not get mixed up and to ensure the anonymity of the participants during data processing, analysis, and publication. Only the participant ID number, not the names of participants, were brought to Carleton University for the data analysis. The consent forms containing the link between participant's name and the participant ID were kept in a locked filing cabinet in the locked office of Dr. Erika Bariciak.

\subsubsection{Data analysis}

The data from the questionnaires and notes recorded were analyzed after the completion of the study. The data from evaluation questionnaires were summarized and frequencies for each of the questions were determined. The observations, task performance and comments, recorded by the study assistant were summarized for a further improvement of the PPADS system.

All materials used in the study can be found in the Appendix D. 


\section{Chapter: Results and Discussion}

\subsection{Neonatal Mortality Prediction Results}

\subsubsection{ANN models results}

This section describes the results achieved in creating neonatal mortality prediction models using summation datasets from the CDR in the NICU at CHEO.

The result files created by the MIRG ANN Builder contain 19 columns; the first 15 represent the options for the network architecture, activation, training and the values of the parameters that drive the learning of the ANN; the next 4 columns show the performance (sensitivity and specificity) results of the network on the test and verification sets, and the last column is the path to the network file. An example of a result file can be found in Appendix B.

\section{ANN models with 12 hour data}

In the fast mode of operation, the average specificity achieved was $95 \%$ and the sensitivity was $47 \%$. There was no significant improvement in the fast mode with the sub-parameter adjustment (adjusting the parameters of the training algorithm): sensitivity $49 \%$ and specificity $95 \%$. In the medium mode, ANN models were able to classify the cases with $85 \%$ sensitivity; yet, this improvement caused a decrease in the specificity, which was $81 \%$. The best results were achieved in the medium mode with the subparameter adjustment: $81 \%$ sensitivity and $95 \%$ specificity, 0.90 AUC, 0.57 PPV, 0.98 NPV, 0.95 ACC, $0.64 \mathrm{MCC}$ and $0.65 \mathrm{~F} 1$-score. The time it took to create the models with 
the best results for the 12 hour data was approximately 3 weeks. The summary of the results for 12 hour data can be found in Table 4-1.

Table 4-1: Results of the classification of 12 hour summation data

\begin{tabular}{|c|c|c|c|c|c|c|c|c|}
\hline Mode & Sens. & Spec. & AUC & PPV & NPV & $\mathbf{A C C}$ & MCC & F1 \\
\hline Fast & $\begin{array}{l}0.47 \pm \\
0.09\end{array}$ & $\begin{array}{l}0.95 \pm \\
0.02\end{array}$ & $\begin{array}{l}0.86 \pm \\
0.07\end{array}$ & $\begin{array}{l}0.39 \pm \\
0.09\end{array}$ & $\begin{array}{l}0.97 \pm \\
0.05\end{array}$ & $\begin{array}{l}0.93 \pm \\
0.02\end{array}$ & $\begin{array}{l}0.39 \pm \\
0.08\end{array}$ & $\begin{array}{l}0.42 \pm \\
0.07\end{array}$ \\
\hline $\begin{array}{l}\text { Fast with } \\
\text { Sub-P }\end{array}$ & $\begin{array}{l}0.49 \pm \\
0.18\end{array}$ & $\begin{array}{l}0.95 \pm \\
0.02\end{array}$ & $\begin{array}{l}0.86 \pm \\
0.08\end{array}$ & $\begin{array}{l}0.39 \pm \\
0.13\end{array}$ & $\begin{array}{l}0.97 \pm \\
0.01\end{array}$ & $\begin{array}{l}0.93 \pm \\
0.02\end{array}$ & $\begin{array}{l}0.39 \pm \\
0.15\end{array}$ & $\begin{array}{l}0.42 \pm \\
0.13\end{array}$ \\
\hline Medium & $\begin{array}{l}0.85 \pm \\
0.00\end{array}$ & $\begin{array}{l}0.81 \pm \\
0.13\end{array}$ & $\begin{array}{l}0.90 \pm \\
0.04\end{array}$ & $\begin{array}{l}0.31 \pm \\
0.19\end{array}$ & $\begin{array}{l}0.98 \pm \\
0.01\end{array}$ & $\begin{array}{l}0.81 \pm \\
0.12\end{array}$ & $\begin{array}{l}0.44 \pm \\
0.18\end{array}$ & $\begin{array}{l}0.42 \pm \\
0.20\end{array}$ \\
\hline $\begin{array}{l}\text { Medium } \\
\text { with Sub-P }\end{array}$ & $\begin{array}{l}0.81 \pm \\
0.16\end{array}$ & $\begin{array}{l}0.95 \pm \\
0.03\end{array}$ & $\begin{array}{l}0.90 \pm \\
0.06\end{array}$ & $\begin{array}{l}0.57 \pm \\
0.21\end{array}$ & $\begin{array}{l}0.98 \pm \\
0.01\end{array}$ & $\begin{array}{l}0.95 \pm \\
0.03\end{array}$ & $\begin{array}{l}0.64 \pm \\
0.16\end{array}$ & $\begin{array}{l}0.65 \pm \\
0.16\end{array}$ \\
\hline \multicolumn{9}{|c|}{ Running Time ${ }^{1}$} \\
\hline \multicolumn{9}{|l|}{ Fast - 1 hour } \\
\hline \multicolumn{9}{|c|}{ Fast with Sub-Parameters - 8 hours } \\
\hline \multicolumn{9}{|c|}{ Medium - 4 days } \\
\hline Medium wit & $\mathrm{b}-\mathrm{P}$ & eters & ppro & weeks & & & & \\
\hline
\end{tabular}

$\underline{\text { ANN models with } 48 \text { hour data }}$

With the fast mode of operation, the ANN models achieved on the average $96 \%$ specificity and $35 \%$ sensitivity. There was an improvement in the sensitivity results for the fast mode with the sub-parameter adjustment: sensitivity $69 \%$ and specificity $95 \%$.

${ }^{1}$ The programs were running on a computer with the following system specifications:

Intel Core i7 $3770(3.4 \mathrm{GHz})$ processor, 8GB DDR3 RAM (800 MHz), Windows 7 64bit. 
In the medium mode, ANN models were able to classify the cases with $72 \%$ sensitivity and $95 \%$ specificity. The results improved further, when the option of the subparameter adjustment was activated for the medium mode: $81 \%$ sensitivity and $98 \%$ specificity, 0.91 AUC, 0.74 PPV, 0.99 NPV, 0.97 ACC, 0.76 MCC and 0.76 F1-score. The running time for the medium mode with sub-parameters was approximately 25 days. The summary of the results for the 48 hour data can be found in Table 4-2.

Table 4-2: Results of the classification of 48 hour summation data

\begin{tabular}{|c|c|c|c|c|c|c|c|c|}
\hline Mode & Sens. & Spec. & AUC & PPV & NPV & ACC & MCC & F1 \\
\hline Fast & $\begin{array}{l}0.35 \pm \\
0.12\end{array}$ & $\begin{array}{l}0.96 \pm \\
0.01\end{array}$ & $\begin{array}{l}0.84 \pm \\
0.06\end{array}$ & $\begin{array}{l}0.38 \pm \\
0.15\end{array}$ & $\begin{array}{l}0.96 \pm \\
0.01\end{array}$ & $\begin{array}{l}0.93 \pm \\
0.01\end{array}$ & $\begin{array}{l}0.32 \pm \\
0.13\end{array}$ & $\begin{array}{l}0.35 \pm \\
0.12\end{array}$ \\
\hline $\begin{array}{l}\text { Fast with } \\
\text { Sub-P }\end{array}$ & $\begin{array}{l}0.69 \pm \\
0.11\end{array}$ & $\begin{array}{l}0.95 \pm \\
0.02\end{array}$ & $\begin{array}{l}0.87 \pm \\
0.06\end{array}$ & $\begin{array}{l}0.47 \pm \\
0.09\end{array}$ & $\begin{array}{l}0.98 \pm \\
0.05\end{array}$ & $\begin{array}{l}0.94 \pm \\
0.01\end{array}$ & $\begin{array}{l}0.54 \pm \\
0.09\end{array}$ & $\begin{array}{l}0.55 \pm \\
0.08\end{array}$ \\
\hline Medium & $\begin{array}{l}0.72 \pm \\
0.06\end{array}$ & $\begin{array}{l}0.95 \pm \\
0.02\end{array}$ & $\begin{array}{l}0.88 \pm \\
0.07\end{array}$ & $\begin{array}{l}0.43 \pm \\
0.12\end{array}$ & $\begin{array}{l}0.98 \pm \\
0.01\end{array}$ & $\begin{array}{l}0.94 \pm \\
0.02\end{array}$ & $\begin{array}{l}0.52 \pm \\
0.10\end{array}$ & $\begin{array}{l}0.53 \pm \\
0.11\end{array}$ \\
\hline $\begin{array}{l}\text { Medium } \\
\text { with Sub-P }\end{array}$ & $\begin{array}{l}0.81 \pm \\
0.09\end{array}$ & $\begin{array}{l}0.98 \pm \\
0.01\end{array}$ & $\begin{array}{l}0.91 \pm \\
0.07\end{array}$ & $\begin{array}{l}0.71 \pm \\
0.14\end{array}$ & $\begin{array}{l}0.99 \pm \\
0.01\end{array}$ & $\begin{array}{l}0.97 \pm \\
0.01\end{array}$ & $\begin{array}{l}0.74 \pm \\
0.09\end{array}$ & $\begin{array}{l}0.74 \pm \\
0.10\end{array}$ \\
\hline \multicolumn{9}{|c|}{ Running Time } \\
\hline \multicolumn{9}{|l|}{ Fast - 1 hour } \\
\hline \multicolumn{9}{|c|}{ Fast with Sub-Parameters - 10 hours } \\
\hline \multicolumn{9}{|c|}{ Medium - Approx. 1week } \\
\hline \multicolumn{9}{|c|}{ Medium with Sub-Parameters - Approx. 25 days } \\
\hline
\end{tabular}


ANN models with 3 attributes (48 hour data)

In the fast mode of operation, the average specificity achieved was $99 \%$ and the sensitivity was $45 \%$. The sensitivity results improved up to $53 \%$ when the sub-parameter adjustment was activated, and the specificity result stayed the same, 99\%. For this dataset, the medium mode gave the best results: the sensitivity improved, reaching $72 \%$, but specificity decreased slightly, down to $96 \%$; the further results for this mode were 0.88 AUC, 0.6 PPV, 0.98 NPV, 0.95 ACC, 0.62 MCC and 0.63 F1-score. When the subparameter adjustment was activated, the sensitivity improved further, up to $86 \%$; however, the specificity decreased to $82 \%$. The summary of the results for 48 hour data with 3 attributes can be found in Table 4-3.

Table 4-3: Results of the classification of 48 hour summation data with 3 attributes

\begin{tabular}{|c|c|c|c|c|c|c|c|c|}
\hline Mode & Sens. & Spec. & $\mathbf{A U C}$ & PPV & NPV & $\mathrm{ACC}$ & MCC & F1 \\
\hline Fast & $\begin{array}{l}0.45 \pm \\
0.19\end{array}$ & $\begin{array}{l}0.99 \pm \\
0.01\end{array}$ & $\begin{array}{l}0.86 \pm \\
0.1\end{array}$ & $\begin{array}{l}0.67 \pm \\
0.16\end{array}$ & $\begin{array}{l}0.97 \pm \\
0.01\end{array}$ & $\begin{array}{l}0.96 \pm \\
0.01\end{array}$ & $\begin{array}{l}0.51 \pm \\
0.14\end{array}$ & $\begin{array}{l}0.50 \pm \\
0.16\end{array}$ \\
\hline $\begin{array}{l}\text { Fast with } \\
\text { Sub-P }\end{array}$ & $\begin{array}{l}0.53 \pm \\
0.15\end{array}$ & $\begin{array}{l}0.99 \pm \\
0.01\end{array}$ & $\begin{array}{l}0.87 \pm \\
0.1\end{array}$ & $\begin{array}{l}0.70 \pm \\
0.19\end{array}$ & $\begin{array}{l}0.97 \pm \\
0.01\end{array}$ & $\begin{array}{l}0.96 \pm \\
0.01\end{array}$ & $\begin{array}{l}0.58 \pm \\
0.13\end{array}$ & $\begin{array}{l}0.59 \pm \\
0.14\end{array}$ \\
\hline Medium & $\begin{array}{l}0.72 \pm \\
0.11\end{array}$ & $\begin{array}{l}0.96 \pm \\
0.04\end{array}$ & $\begin{array}{l}0.88 \pm \\
0.03\end{array}$ & $\begin{array}{l}0.60 \pm \\
0.2\end{array}$ & $\begin{array}{l}0.98 \pm \\
0.00\end{array}$ & $\begin{array}{l}0.95 \pm \\
0.01\end{array}$ & $\begin{array}{l}0.62 \pm \\
0.13\end{array}$ & $\begin{array}{l}0.63 \pm \\
0.15\end{array}$ \\
\hline $\begin{array}{l}\text { Medium } \\
\text { with Sub-P }\end{array}$ & $\begin{array}{l}0.86 \pm \\
0.00\end{array}$ & $\begin{array}{l}0.82 \pm \\
0.13\end{array}$ & $\begin{array}{l}0.89 \pm \\
0.08\end{array}$ & $\begin{array}{l}0.24 \pm \\
0.2\end{array}$ & $\begin{array}{l}0.99 \pm \\
0.00\end{array}$ & $\begin{array}{l}0.82 \pm \\
0.12\end{array}$ & $\begin{array}{l}0.39 \pm \\
0.10\end{array}$ & $\begin{array}{l}0.36 \pm \\
0.10\end{array}$ \\
\hline $\begin{array}{l}\text { Running Ti } \\
\text { Fast - } 12 \mathrm{mir} \\
\text { Fast with } \mathrm{Su}\end{array}$ & $\begin{array}{l}\text { le } \\
\text { ates } \\
\text {-Param }\end{array}$ & $s-11$ & & & & & & \\
\hline
\end{tabular}


Medium - 5 hours

Medium with Sub-Parameters - 5 days

Comparison to the previous work

The results of 48 hour summation data with 18 and 3 attributes were compared to the previous work; only sensitivity and specificity values were calculated in the previous work and those values are presented here. The results of the dataset with 18 attributes were higher than the MIRG ANN RFW results by $13 \%$ for the sensitivity and by $1 \%$ for the specificity. For the dataset with 3 attributes the sensitivity was higher by $10 \%$, but specificity was lower by $3 \%$.

Table 4-4: MIRG ANN RFW vs. MIRG ANN Builder

\begin{tabular}{|c|c|c|c|c|}
\hline & \multicolumn{2}{|c|}{48 hour set } & 48 hour with 3 attributes \\
\hline & Sensitivity & Specificity & Sensitivity & Specificity \\
\hline MIRG ANN RFW & $68 \pm 14$ & $97 \pm 3$ & $62 \pm 14$ & $99 \pm 1$ \\
(Gilchrist, 2012) & & & & \\
\hline MIRG ANN Builder & $81 \pm 17$ & $98 \pm 1$ & $72 \pm 11$ & $96 \pm 4$ \\
\hline
\end{tabular}




\subsubsection{Integration with the PPADS tool}

The Connection Tool was able calculate the mortality predictions for 20 patients, and pass those estimates to the databases the PPADS tool in a mean of 0.48 seconds, which meets the requirement of providing results in real-time (within one minute). Figure 4-1 illustrates the filled-in patients_predicted table in the CDSS database.

\begin{tabular}{|c|c|c|c|c|c|c|c|}
\hline PatientID & StudyID & Timestamp & PredictionName & PredictionID & PredictionOutcome & PredictionScore & PredictionRiskLeve \\
\hline 900011 & 2 & Thu Nov 20 ... & Mortality & 1 & 1 & 0.976606 & High \\
\hline 900001 & 37 & Thu Nov $20 \ldots$ & Mortality & 1 & -1 & -0.981682 & Low \\
\hline 900002 & 48 & Thu Nov 20 ... & Mortality & 1 & -1 & -0.982748 & Low \\
\hline 900003 & 63 & Thu Nov 20 ... & Mortality & 1 & -1 & -0.990676 & Low \\
\hline 900012 & 65 & Thu Nov 20 ... & Mortality & 1 & -1 & -0.9923 & Low \\
\hline 900004 & 121 & Thu Nov 20 ... & Mortality & 1 & -1 & -0.968128 & Low \\
\hline 900006 & 170 & Thu Nov 20 ... & Mortality & 1 & -1 & -0.988476 & Low \\
\hline 900007 & 171 & Thu Nov 20 ... & Mortality & 1 & -1 & -0.990689 & Low \\
\hline 900009 & 227 & Thu Nov 20 ... & Mortality & 1 & -1 & -0.992763 & Low \\
\hline 900016 & 229 & Thu Nov 20 ... & Mortality & 1 & -1 & -0.989321 & Low \\
\hline 900017 & 230 & Thu Nov 20 ... & Mortality & 1 & -1 & -0.990419 & Low \\
\hline 900019 & 230 & Thu Nov $20 \ldots$ & Mortality & 1 & -1 & -0.990419 & Low \\
\hline 900013 & 342 & Thu Nov 20 ... & Mortality & 1 & 1 & 0.931159 & High \\
\hline 900014 & 401 & Thu Nov 20 ... & Mortality & 1 & -1 & -0.961703 & Low \\
\hline 900018 & 511 & Thu Nov 20 ... & Mortality & 1 & -1 & -0.993163 & Low \\
\hline 900020 & 513 & Thu Nov 20 ... & Mortality & 1 & -1 & -0.993901 & Low \\
\hline 900010 & 514 & Thu Nov 20 ... & Mortality & 1 & -1 & -0.990086 & Low \\
\hline 900015 & 515 & Thu Nov $20 \ldots$ & Mortality & 1 & -1 & -0.99193 & Low \\
\hline 900008 & 824 & Thu Nov 20 ... & Mortality & 1 & -1 & -0.968995 & Low \\
\hline 900005 & 825 & Thu Nov 20 ... & Mortality & 1 & -1 & -0.98255 & Low \\
\hline
\end{tabular}

Figure 4-1: The patients_predictions table in the CDSS database

Additionally, the information about the model, such as the sensitivity, the specificity, and the AUC value were added the predictions_info table as shown in Figure $4-2$.

\begin{tabular}{|c|c|c|c|c|c|c|}
\hline PredictionID & PredictionNa... & PredictionDescription & PredictionSensitivity & PredictionSpecificity & PredictionAUC \\
\hline & 1: & Mortality & Predicting the outcome ... & 75 & 98 & 0.96 \\
\hline
\end{tabular}

Figure 4-2: The predictions_info table in the CDSS database 


\subsubsection{Summary of the mortality prediction results}

By testing several operational models of the MIRG ANN Builder, prediction models were created for 12 hour and 48 hour datasets (with 18 and 3 attributes). Since we wanted to compare the performance of new models to the previous work, and the latter were optimized according to the sensitivity and specificity, those two performance measures were chosen as targets for optimizing the ANN parameters and the performance. The highest average results for the 12 hour data were $81 \%$ sensitivity and $95 \%$ specificity, for the 48 hour data $81 \%$ sensitivity and $98 \%$, and for the 48 hour data with 3 attributes $72 \%$ sensitivity and $96 \%$ specificity. All results exceeded the usefulness criteria.

The ANN models were integrated with the PPADS tool to present the risk of mortality for patients accepted to the NICU. The Connection Tool retrieved the list of active patients from the CDSS database, queried data from the CDR database on a local machine, used the ANN models to calculate the predictions and stored these predictions back to the CDSS database in a mean of 0.48 seconds for 20 patients. This short running time will ensure a quick computation and display of prediction results could be available when there is a demand, for example when clinicians review the patient cases and need to see the latest, most up to date information; otherwise, the predictions will be updated during the regular updates of the PPADS tool, which is once or twice per day.

In order to automate the process of data preparation, and the selection of best performing networks from results files, several tools have been created using BASH scripting. This automation can be used in scheduling regular updates of the prediction models that are integrated with the PPADS tool. 


\subsection{Pilot Study Results}

Two parents were recruited for the short pilot study of the PPADS tool. The summary of questionnaires and other qualitative data (quotes, comments and suggestion) are presented in this section.

\subsubsection{Background questionnaire summary}

Both participants were female, belonged to the age group of 30-39, had a university/collage degree, and during their neonate's stay in the NICU were presented with the withdrawal of care option. Computer skill levels specified by the participants were: Participant \#1, advanced skill level, and Participant \#2, expert skill level.

\subsubsection{Evaluation questionnaire summary}

The questions were evaluated on a four (or a three) point Likert scales. On this scale, 4 (or 3 ) is the highest score and 1 is the lowest.

\section{$\underline{\text { Usefulness }}$}

Both participants strongly agreed that the list of neonate's medical condition, the list of current treatments, and the risk predictions were useful.

Both participants agreed or strongly agreed that the direction of care options were presented in a balanced way and the glossary definitions and the reason a change in direction of care was suggested were useful. 
The opinions of the participants regarding the usefulness of the decision support form, which was intended to help parents decide between different direction of care options, varied: one of the participants agreed, while the other disagreed.

To the statement, that after using the PPADS tool participants would feel more informed, would better understand their decisional options, would feel more certain about their decision and feel clearer about how their values affect their decisions, one of the participants agreed and the other participant disagreed.

Lastly, one of the participants agreed that they would use the PPADS tool if given the opportunity, while the other participant disagreed. The summary of the usefulness evaluation is shown in Table 4-5.

Table 4-5: Usefulness results

\begin{tabular}{|c|l|c|}
\hline \multirow{4}{*}{ Questions } & \multicolumn{2}{|c|}{ Results } \\
\cline { 2 - 3 } The list of neonate's current medical & \multicolumn{1}{|c|}{ Likert option } & Frequency \\
condition was useful (Q10-1) & (4) Strongly Agree & 2 \\
\cline { 2 - 3 } & (3) Agree & 0 \\
\cline { 2 - 3 } & (2) Disagree & 0 \\
\cline { 2 - 3 } The list of neonate's current treatments & (1) Strongly Disagree & 0 \\
\hline \multirow{4}{*}{$\begin{array}{c}\text { was useful (Q10-3) } \\
\text { The risk predictions were useful(Q10- }\end{array}$} & (4) Strongly Agree & 2 \\
\cline { 2 - 3 } 5) & (3) Agree & 0 \\
\cline { 2 - 3 } & (2) Disagree & 0 \\
\cline { 2 - 3 } & (1) Strongly Disagree & 0 \\
\hline & (4) Strongly Agree & 2 \\
\cline { 2 - 3 } & (2) Disagree & 0 \\
\cline { 2 - 3 } & (1) Strongly Disagree & 0 \\
\hline The reason a change in direction of care & (4) Strongly Agree & 1 \\
\hline
\end{tabular}




\begin{tabular}{|c|c|c|}
\hline & (3) Agree & 1 \\
\hline & (2) Disagree & 0 \\
\hline & (1) Strongly Disagree & 0 \\
\hline \multirow{4}{*}{$\begin{array}{l}\text { The decision support form was useful } \\
\text { (Q10-9) }\end{array}$} & (4) Strongly Agree & 1 \\
\hline & (3) Agree & 0 \\
\hline & (2) Disagree & 1 \\
\hline & (1) Strongly Disagree & 0 \\
\hline \multirow{4}{*}{$\begin{array}{l}\text { The directions of care options were } \\
\text { presented in a balanced way (Q10-11) }\end{array}$} & (4) Strongly Agree & 1 \\
\hline & (3) Agree & 1 \\
\hline & (2) Disagree & 0 \\
\hline & (1) Strongly Disagree & 0 \\
\hline \multirow{4}{*}{$\begin{array}{l}\text { The glossary definitions were useful } \\
\qquad(\mathrm{Q} 10-12)\end{array}$} & (4) Strongly Agree & 1 \\
\hline & (3) Agree & 1 \\
\hline & (2) Disagree & 0 \\
\hline & (1) Strongly Disagree & 0 \\
\hline \multirow{4}{*}{$\begin{array}{l}\text { After using PPADS would feel more } \\
\text { informed } \\
\text { (Q10-14) }\end{array}$} & (4) Strongly Agree & 1 \\
\hline & (3) Agree & 0 \\
\hline & (2) Disagree & 1 \\
\hline & (1) Strongly Disagree & 0 \\
\hline \multirow{4}{*}{$\begin{array}{l}\text { After using PPADS would have better } \\
\text { understanding of decisional options } \\
\text { (Q10-15) }\end{array}$} & (4) Strongly Agree & 1 \\
\hline & (3) Agree & 0 \\
\hline & (2) Disagree & 1 \\
\hline & (1) Strongly Disagree & 0 \\
\hline \multirow{4}{*}{$\begin{array}{l}\text { After using PPADS would feel more } \\
\text { certain about decision } \\
\text { (Q10-16) }\end{array}$} & (4) Strongly Agree & 1 \\
\hline & (3) Agree & 0 \\
\hline & (2) Disagree & 1 \\
\hline & (1) Strongly Disagree & 0 \\
\hline \multirow{4}{*}{$\begin{array}{l}\text { After using PPADS feel clearer about } \\
\text { how values affect decision } \\
(\mathrm{Q} 10-17)\end{array}$} & (4) Strongly Agree & 1 \\
\hline & (3) Agree & 0 \\
\hline & (2) Disagree & 1 \\
\hline & (1) Strongly Disagree & 0 \\
\hline \multirow{2}{*}{$\begin{array}{l}\text { If given opportunity participant would } \\
\text { use the tool }\end{array}$} & (4) Strongly Agree & 1 \\
\hline & (3) Agree & 0 \\
\hline
\end{tabular}




$$
\text { (Q10-18) }
$$

\begin{tabular}{|l|l|}
\hline (2) Disagree & 1 \\
\hline (1) Strongly Disagree & 0 \\
\hline
\end{tabular}

\section{$\underline{\text { Efficiency and Effectiveness }}$}

Both parents strongly agreed that the information was clearly organized, the meanings of pages and buttons were easy to understand, performing the tasks was easy, the tool would be easy to learn, using the tool would not add stress and it would be easy for users to find the information they are looking for.

Both parents agreed or strongly agreed that the tool can be navigated easily and quickly.

The results of the efficiency and effectiveness of the tool can be found in Table 4-6.

Table 4-6: Efficiency and effectiveness results

\begin{tabular}{|c|l|c|}
\hline \multirow{2}{*}{ Questions } & \multicolumn{2}{|c|}{ Results } \\
\cline { 2 - 3 } $\begin{array}{c}\text { The information was clearly organized } \\
\text { (Q2) }\end{array}$ & \multicolumn{1}{|c|}{ Likert option } & Frequency \\
\cline { 2 - 3 } & (4) Very Clear & 2 \\
\cline { 2 - 3 } & (3) Moderately Clear & 0 \\
\cline { 2 - 3 } & (2) Some Confusing & 0 \\
\cline { 2 - 3 } Navigation was easy & (1) Very Confusing & 0 \\
\hline \multirow{2}{*}{$(\mathrm{Q} 3)$} & (4) Very Easy & 1 \\
\cline { 2 - 3 } & (3) Moderately Easy & 1 \\
\cline { 2 - 3 } & (2) Some Difficult & 0 \\
\cline { 2 - 3 } & (1) Very Difficult & 0 \\
\hline Meaning of pages and buttons were & (4) Very Easy & 2 \\
\hline
\end{tabular}




\begin{tabular}{|c|c|c|}
\hline & (3) Moderately Easy & 0 \\
\hline & (2) Some Difficult & 0 \\
\hline & (1) Very Difficult & 0 \\
\hline \multirow{4}{*}{$\begin{array}{l}\text { Performing the tasks was easy } \\
\text { (Q5) }\end{array}$} & (4) Always Easy & 2 \\
\hline & (3) Mostly Easy & 0 \\
\hline & (2) Sometimes Easy & 0 \\
\hline & (1) Never Easy & 0 \\
\hline \multirow{4}{*}{ The tool would be easy to learn (Q9-1) } & (4) Strongly Agree & 2 \\
\hline & (3) Agree & 0 \\
\hline & (2) Disagree & 0 \\
\hline & (1) Strongly Disagree & 0 \\
\hline \multirow{4}{*}{$\begin{array}{l}\text { Using the tool would not add stress } \\
\qquad \text { (Q9-2) }\end{array}$} & (4) Strongly Agree & 2 \\
\hline & (3) Agree & 0 \\
\hline & (2) Disagree & 0 \\
\hline & (1) Strongly Disagree & 0 \\
\hline \multirow{4}{*}{$\begin{array}{l}\text { It was easy to find the information they } \\
\text { were looking for (Q9-3) }\end{array}$} & (4) Strongly Agree & 2 \\
\hline & (3) Agree & 0 \\
\hline & (2) Disagree & 0 \\
\hline & (1) Strongly Disagree & 0 \\
\hline \multirow{4}{*}{$\begin{array}{l}\text { The tool could be navigated quickly } \\
\qquad(\mathrm{Q} 9-4)\end{array}$} & (4) Strongly Agree & 1 \\
\hline & (3) Agree & 1 \\
\hline & (2) Disagree & 0 \\
\hline & (1) Strongly Disagree & 0 \\
\hline
\end{tabular}

\section{Acceptability}

Both participants found the amount of information in the PPADS tool to be "just right". The length of the decision support form was found to be "just right" by one of the participants while the other participant did not answer the question. 
Both participants indicated that the terminology used in the PPADS tool was moderately easy to understand.

Both participants agreed or strongly agreed that the decision support form, the list of current medical conditions, the list of current treatments, the risk predictions, the reason of a change in direction of the care was suggested, and the glossary definitions were easy to understand.

All three tasks were successfully completed by the participants. The results regarding the acceptability of the tool are summarized in Table 4-7.

Table 4-7: Acceptability results

\begin{tabular}{|c|c|c|}
\hline \multirow{2}{*}{ Questions } & \multicolumn{2}{|c|}{ Results } \\
\hline & Likert option & Frequency \\
\hline \multirow{3}{*}{$\begin{array}{l}\text { Amount of information in PPADS was } \\
\text { good } \\
\text { (Q6) }\end{array}$} & (3) Too much & 0 \\
\hline & (2) Just right & 2 \\
\hline & (1) Too little & 0 \\
\hline \multirow{3}{*}{$\begin{array}{l}\text { The length of the decision support form is } \\
\text { good } \\
\text { (Q7) }\end{array}$} & (3) Too much & 0 \\
\hline & (2) Just right & 1 \\
\hline & (1) Too little & 0 \\
\hline \multirow{4}{*}{$\begin{array}{l}\text { The terms used in PPADS were easy to } \\
\text { understand } \\
\text { (Q8) }\end{array}$} & $\begin{array}{l}\text { (4) Always easily } \\
\text { understood }\end{array}$ & 0 \\
\hline & $\begin{array}{l}\text { (3) Moderately easily } \\
\text { understood }\end{array}$ & 2 \\
\hline & $\begin{array}{l}\text { (2) Sometimes easily } \\
\text { understood }\end{array}$ & 0 \\
\hline & $\begin{array}{l}\text { (1) Never easily } \\
\text { understood }\end{array}$ & 0 \\
\hline The list of current medical conditions was & (4) Strong Agree & 2 \\
\hline
\end{tabular}




\begin{tabular}{|c|c|c|}
\hline & (3) Agree & 0 \\
\hline & (2) Disagree & 0 \\
\hline & (1) Strong Disagree & 0 \\
\hline \multirow{4}{*}{$\begin{array}{l}\text { The list of the current medical conditions } \\
\text { was easy to understand } \\
\text { (Q10-4) }\end{array}$} & (4) Strong Agree & 1 \\
\hline & (3) Agree & 1 \\
\hline & (2) Disagree & 0 \\
\hline & (1) Strong Disagree & 0 \\
\hline \multirow{4}{*}{$\begin{array}{l}\text { The risk predictions were easy to } \\
\text { understand } \\
\text { (Q10-6) }\end{array}$} & (4) Strong Agree & 2 \\
\hline & (3) Agree & 0 \\
\hline & (2) Disagree & 0 \\
\hline & (1) Strong Disagree & 0 \\
\hline \multirow{4}{*}{$\begin{array}{l}\text { The reason a change in direction of care } \\
\text { was suggested was easy to understand } \\
\text { (Q10-8) }\end{array}$} & (4) Strong Agree & 1 \\
\hline & (3) Agree & 1 \\
\hline & (2) Disagree & 0 \\
\hline & (1) Strong Disagree & 0 \\
\hline \multirow{4}{*}{$\begin{array}{l}\text { The decision support form was easy to } \\
\text { understand (Q10-10) }\end{array}$} & (4) Strong Agree & 1 \\
\hline & (3) Agree & 1 \\
\hline & (2) Disagree & 0 \\
\hline & (1) Strong Disagree & 0 \\
\hline \multirow{4}{*}{$\begin{array}{l}\text { The glossary definitions were easy to } \\
\text { understand } \\
\text { (Q10-12) }\end{array}$} & (4) Strong Agree & 1 \\
\hline & (3) Agree & 1 \\
\hline & (2) Disagree & 0 \\
\hline & (1) Strong Disagree & 0 \\
\hline Task completion & \multicolumn{2}{|c|}{$\begin{array}{l}\text { Both participants successfully completed all } \\
\text { three tasks. }\end{array}$} \\
\hline
\end{tabular}

$\underline{\text { Satisfaction }}$

Both parents found the PPADS tool to be satisfying to use as shown in the results summed up in Table 4-8. 
Table 4-8: Satisfaction results

\begin{tabular}{|c|l|c|}
\hline \multirow{2}{*}{ Questions } & \multicolumn{2}{|c|}{ Results } \\
\cline { 2 - 3 } & \multicolumn{1}{|c|}{ Likert option } & $\begin{array}{c}\text { Participant } \\
\text { Frequency }\end{array}$ \\
\hline \multirow{3}{*}{$\begin{array}{c}\text { The PPADS tool was satisfying to use } \\
\text { (Q1) }\end{array}$} & (4) Exceptional & 0 \\
\cline { 2 - 3 } & (3) Satisfying & 2 \\
\cline { 2 - 3 } & (2) Frustrating & 0 \\
\cline { 2 - 3 } & (1) Unacceptable & 0 \\
\hline
\end{tabular}

\subsubsection{Qualitative data summary}

Participant quotes during their interaction with the PPADS tool, and their impressions expressed afterwards were recorded and are presented in the tables 4-9 to 4-11.

Table 4-9: Positive quotes

\begin{tabular}{|c|l|}
\hline Participant ID & \multicolumn{1}{|c|}{ Positive Quote } \\
\hline $\mathbf{9 2 8 3 8 0}$ & Information is good. \\
\hline $\mathbf{9 2 8 3 8 0}$ & Note from a doctor is good. \\
\hline $\mathbf{9 2 8 3 8 0}$ & Outcome prediction is good. \\
\hline $\mathbf{7 5 2 6 3 7}$ & Rich text, good for learning the medical terminology. \\
\hline $\mathbf{7 5 2 6 3 7}$ & Glossary works. \\
\hline $\mathbf{7 5 2 6 3 7}$ & $\begin{array}{l}\text { I cannot ask for my child's file from physicians, and this tool presents the } \\
\text { information that CHEO wants parents to know right here. }\end{array}$ \\
\hline
\end{tabular}


Table 4-10: Negative quotes

\begin{tabular}{|c|l|}
\hline Participant ID & \multicolumn{1}{c|}{ Negative Quote } \\
\hline $\mathbf{9 2 8 3 8 0}$ & To make a choice I want to speak to a person not a computer. \\
\hline $\mathbf{9 2 8 3 8 0}$ & $\begin{array}{l}\text { Not sure if it (the decision support form) would make me make a } \\
\text { decision. It is more definitions than an advice. I feel like this helps to } \\
\text { understand the different steps of care but not in making the decision. }\end{array}$ \\
\hline $\mathbf{7 5 2 6 3 7}$ & Term definitions are given few times. \\
\hline
\end{tabular}

Table 4-11: Suggestions

\begin{tabular}{|c|l|}
\hline Participant ID & \multicolumn{1}{c|}{ Suggestion } \\
\hline $\mathbf{9 2 8 3 8 0}$ & Add information what other parents did in similar situation. \\
\hline $\mathbf{9 2 8 3 8 0}$ & $\begin{array}{l}\text { Next to outcome predictions add for example 85\% of parents in this } \\
\text { situation did this. }\end{array}$ \\
\hline $\mathbf{9 2 8 3 8 0}$ & $\begin{array}{l}\text { Say it more often, what is the reason a change in direction of care was } \\
\text { suggested. }\end{array}$ \\
\hline $\mathbf{9 2 8 3 8 0}$ & Add doctor's and nurse's point of view. \\
\hline $\mathbf{7 5 2 6 3 7}$ & Clarify that the decision support form is optional to fill in, and \\
& explain when it is going to be used. \\
\hline $\mathbf{7 5 2 6 3 7}$ & Add a tab for completed decision support forms. \\
\hline
\end{tabular}




\subsubsection{Summary of the pilot study results}

In order to understand how acceptable the PPADS tool would be for users who are in the stressful environment of the NICU and are under the added stress of contemplating the difficult option of withdrawing life sustaining treatment for their critically ill infant, we conducted a study with parents whose infant died in the NICU, as they have been through a similar situation. Both parents indicated that the current condition, treatments and outcome predictions were useful and easy to understand. One of the participants also commented that parents cannot ask for their child's file from clinicians, and the PPADS tool provides all the necessary information that physicians want parents to know.

Regarding the usefulness of the decision support form, one of the participants indicated that the tool would not have helped them in arriving at the decision and they would not want to fill in a form during the stressful time of their neonate's stay in the NICU. Since the components of the parent interface (conditions, treatments, predictions, and the decision support) are activated by clinician users only when the healthcare team finds that the decision support form, or any other component of the tool, may be useful to parents, the tool is not intended to be used by every parent in the NICU. In addition, since each of the research participants had a unique experience with the death of their baby, this may have also influenced their perception of how useful the tool would be in a subsequent scenario.

The suggestions that parents provided are important for the further improvement of the PPADS tool. For instance, the suggestion to add a tab for completed decision support forms was made. The participant commented that in the stressful and confusing 
environment of the NICU, even scrolling down to the bottom of the page to open the forms would have been inconvenient for them. Having an easily accessible tab to quickly find and print the forms would have been more useful. Another suggestion was, to clarify to users that the decision support form is not mandatory but optional. When the PPADS tool is to be used in a real life situation, parents need to be clear that the tool is an adjunct to discussions held with the medical team and will only be used after the initial team meeting with the parents has occurred, if the family wishes to use it, in order for parents to better understand their neonate's situation and options. 


\section{Chapter: Conclusions}

\subsection{Final Remarks}

In our effort to classify neonatal mortality outcomes, an automated tool was designed and developed for building ANNs. MIRG ANN Builder, using functions from an open source ANN library, automatically adjusts the options and parameter values of feed-forward ANNs in order to create various models. With the MIRG ANN Builder, mortality models with the average of $81 \%$ sensitivity and $98 \%$ specificity results were developed using 48 hour summation data. The results of the models, developed and selected according to their sensitivity and specificity performance, exceeded the clinically usefulness criteria (Sensitivity $\geq 60$, Specificity $\geq 90$ ) and the results of the previous ANN application (Sensitivity $=68$, Specificity $=97$ ). Since the data we are using to create ANN models come from a non-homogenous population: CHEO NICU patients are out-born and are admitted at various ages during their first month of life, we expect to have better results when using data from patients who were born and stayed the first days of life within the same hospital unit. Additionally, before the prediction models will be used in clinical practice, we will need to optimize the ANN parameters and the network selection process according to other performance measures such as accuracy or positive predictive value (PPV). The MIRG ANN Builder can be easily adjusted to perform in this manner; however the level of accuracy or the PPV reached will be limited by the low prevalence of death in this population of infants.

In order to provide real-time (within one minute) prediction about the risk of the mortality for neonates admitted to the NICU, the mortality models were integrated with 
the PPADS system, a clinical decision support system for physicians and parents. The program, developed for accomplishing the integration, was able to query medical data, use the model for classification and write the predictions into the CDSS database of the PPADS system in a mean of 0.48 seconds. With this, the first objective of the thesis: creating clinically useful mortality models, and integrating these with the PPADS tool was completed.

The PPADS tool was evaluated for its usefulness through a short, but ongoing pilot study involving two parents so far whose child died in the NICU at CHEO. The opinions of recruited participants are important in evaluating the different components of the PPADS tool. The feedback participants provided will help to decide how the system can be best integrated in the NICU in order to minimize added stress for parents when providing them with computerized decision support. The summary of the evaluation questionnaire indicated that parents found the information about medical condition, treatment and outcome predictions to be understandable and useful. The opinions of parents about the decision support form for direction of care change were different: One of the participants indicated their strong agreement that the tool will help them make decisions while the other parent disagreed. The feedback of only two participants does not allow us to make conclusions regarding the usefulness and acceptability of the tool; however, the results are encouraging, especially when we consider the useful comments made by both parents. Various components of the tool are separated and are only activated by the healthcare team when deemed appropriate for a given parent, thus it is customizable for each individual patient. With this design, the extent of the support provided by the PPADS tool can be adjusted to best suit the needs of parents in the 
NICU. The second objective of conducting a pilot study to evaluate the PPADS tool was completed.

\subsection{Contributions to Knowledge}

1. The design and the implementation of the MIRG ANN Builder improved the development of outcome prediction models by automating the adjustment of ANN options and parameter values. Using four different operational models of the tool, mortality models have been created with 12 hour, 48 hour and 48 hour with 3 attributes datasets; the results exceeded the criteria for clinical usefulness and gave better results compared to the previous ANN tool.

2. A set of automated tools have been created to simplify the process of data preparation, network selection and performance measure calculation. With MIRG ANN Builder, these make a package of data mining tools that can be used by researchers in our group to create prediction models for different clinical outcomes and different types of data.

3. The prediction models have been integrated into the PPADS system to provide real-time (within a minute) predictions regarding the risk of mortality of NICU patients.

4. A short pilot study was conducted to test the usability of the PPADS tool with parents who have been through the stressful environment of the NICU, and have faced the difficult decision of withdrawing the life support for their neonate. Results indicate that participants found the tool to be easy to navigate, the information provided by the PPADS tool to be well organized, 
understandable and useful. The suggestions that participants provided are important for the improvement of the tool, to make it more suitable for integration in the NICU.

\subsection{Future Work}

1. Mortality prediction models

a) Testing the slow mode of operation.

b) Analyzing the result files created after the operational run of the MIRG ANN Builder. This will give insights regarding the similarity of parameters on different cross validation sets and about the parameter values and options that contribute to the optimal performance of networks.

c) Adding a different stopping criterion for the ANN learning: the current criterion is to stop the training when the desired MSE is reached. The logarithmic sensitivity criterion from previous works can be tested for its performance with the MIRG ANN Builder.

d) Optimizing ANN performances with other performance measures (e.g. accuracy and PPV).

e) Testing deep neural networks (more hidden layers) and/or other types of ANNs such as recurrent networks.

f) Develop a new system for replacing missing values with real-time data streaming. 
g) Testing the models with a more homogenous and generalizable population of infants (i.e. infants born at the treating hospital and admitted on the first day of life.)

2. Integration of the models

a) Testing the performance of the Connection Tool at CHEO with real-time CDR.

b) Extending the functionality of the Connection Tool to automatically fill in the admission information of patients when a new patient account is created.

3. Improving the PPADS system

a) Adding alert generation for clinicians if there is a sudden change in the risk of mortality to the higher level of risk.

b) Implementing the suggestions for improvements from the pilot study. 


\section{References}

(Allen et al., 1993) Allen, M. C., Donohue, P. K., and Dusman, A. E. (1993). The limit of viability: neonatal outcome of infants born at 22 to 25 weeks' gestation. New England Journal of Medicine, 329(22), 1597-1601.

Anand, K. J., Stevens, B. J., and McGrath, P. J. (Eds.). (2007). Pain in neonates and infants. Elsevier Health Sciences.

(Bariciak, 2014) Personal communications from Dr. Erika Barciak.

(Becker and Grunwald, 2000) Becker, P. T. and Grunwald, P. C. (2000). Contextual dynamics of ethical decision making in the NICU. The Journal of perinatal \& neonatal nursing, 14(2), 58-72.

(Berner 2007, 4-8) Berner, E. S. Clinical Decision Support Systems (2007). New York: Springer Science and Business Media, LLC, Chapter 1, pp 4-8.

Blackburn, S. (1982). The neonatal ICU: A high-risk environment. AJN The American Journal of Nursing, 82(11), 1708-1712.

(Blount et al., 2010) Blount, M., Ebling, M. R., Eklund, J. M., James, A. G., McGregor, C., Percival, N., and Sow, D. (2010). Real-time analysis for intensive 
care: development and deployment of the Artemis analytic system. Engineering in Medicine and Biology Magazine, IEEE, 29(2), 110-118.

(Brazy et al., 2001) Brazy, J. E., H. Anderson, B. M., Becker, P. T., and Becker, M. (2001). How parents of premature infants gather information and obtain support. Neonatal Network: The Journal of Neonatal Nursing, 20(2), 41-48.

Bunch, E. H. (2002). High technology and nursing: ethical dilemmas nurses and physicians face on high-technology units in Norway. Nursing Inquiry, 9(3), 187-195.

(CIHI, 2006) Canadian Institute for Health Information (CIHI) (2006). Giving Birth in Canada: The Cost. Available at: https://secure.cihi.ca/free_products/Costs_Report_06_Eng.pdf. Accessed November $20,2014$.

(CIHI, 2009) Canadian Institute for Health Information (CIHI) (2009). Too Early, Too Small: A Profile of Small Babies Across Canada. Available at: https://secure.cihi.ca/free_products/too_early_too_small_en.pdf. Accessed November $20,2014$.

(Cloherty et al., 2008) Cloherty, J. P., Eichenwald, E. C., and Stark, A. R. (Eds.). (2008). Manual of neonatal care. Lippincott Williams \& Wilkins. 
(Coulter and Collins, 2011) Coulter, A., and Collins, A. (2011). Making shared decision-making a reality. London: King's Fund.

(Dietterich, 1998) Dietterich, T. G. (1998). Approximate statistical tests for comparing supervised classification learning algorithms. Neural computation, 10(7), 1895-1923.

(Duda et al. 2012, 288-296) Duda, R. O., Hart, P. E., and Stork, D. G. (2012). Pattern classification. John Wiley \& Sons, Chapter 6, pp 288-296.

(Eden et al., 2010) Eden, L. M. and Callister, L. C. (2010). Parent involvement in end-of-life care and decision making in the newborn intensive care unit: an integrative review. The Journal of perinatal education, 19(1), 29.

(Ennett et al., 2001) Ennett, C. M., M. Frize, and C. R. Walker (2001). Influence of missing values on artificial neural network performance. Medinfo 10 (1), 449-453.

(Ennett et al., 2002) Ennett, C. M., Frize, M., and Scales, N. (2002, October). Logarithmic-sensitivity index as a stopping criterion for neural networks. In Proceedings of 24th Annual Conference of the Engineering in Medicine and Biology and the Annual Fall Meeting of the Biomedical Engineering Society, pp 2326. 
(Ennett, 2003) Ennett, C. M., \& Frize, M. (2003, September). Validation of a hybrid approach for imputing missing data. In Engineering in Medicine and Biology Society, 2003. Proceedings of the 25th Annual International Conference of the IEEE (Vol. 2, pp. 1268-1271).

(Ennett et al., 2004) Ennett, C. M., Frize, M., and Charette, E. (2004). Improvement and automation of artificial neural networks to estimate medical outcomes. Medical engineering and physics, 26(4), 321-328.

(Ennett et al., 2008) Ennett, C. M., Frize, M., and Walker, C. R. (2008, August). Imputation of missing values by integrating neural networks and case-based reasoning. In Engineering in Medicine and Biology Society, 2008. EMBS 2008. 30th Annual International Conference of the IEEE, pp 4337-4341.

(FANN, 2014) Fast Artificial Neural Network library documentation (FANN). Available at: http://leenissen.dk/fann/wp. Accessed November 17, 2014.

(Fawcett, 2006) Fawcett, T. (2006). An introduction to ROC analysis. Pattern recognition letters, 27(8), 861-874.

(Frize and Walker, 2000) Frize, M. and Walker, R. (2000). Clinical decision-support systems for intensive care units using case-based reasoning. Medical engineering $\&$ physics, 22(9), 671-677. 
(Frize et al., 2005) Frize, M., Yang, L., Walker, R. C., and O'Connor, A. M. (2005). Conceptual framework of knowledge management for ethical decision-making support in neonatal intensive care. Information Technology in Biomedicine, IEEE Transactions on, 9(2), 205-215.

(Frize 2011, 51-52) Frize, M. (2011). Ethics for Bioengineers. Synthesis Lectures on Biomedical Engineering, 6(3), Chapter 5, pp 51-52.

(Frize et al., 2011) Frize, M., Weyand, S., Bariciak, E., Gilchrist, J., Dunn, S., and Tozer, S. (2011, May). Combined Physician-Parent Decision Support tool for the neonatal intensive care unit. In Medical Measurements and Applications Proceedings (MeMeA), 2011 IEEE International Workshop on, pp 59-64.

(Frize et al., 2013) Frize M, Bariciak E, and Gilchrist J (2013) PPADS: PhysicianPArent Decision-Support for Neonatal Intensive Care, MEDINFO Proc. vol. 192, World Congress on Medical and Health Informatics, Copenhagen, Denmark, 2013, pp 23-27.

(Gilchrist et al., 2010) Gilchrist, J., M. Frize, C, M. Ennett, and E. Bariciak (2010, April). Performance evaluation of various storage formats for clinical data repositories. In Proceedings of the 2010 IEEE International Workshop on Medical Measurements and Applications, pp 63-68. 
(Gilchrist et al., 2011) Gilchrist, J., Frize, M., Ennett C.M. and Bariciak E. (2011). Neonatal mortality prediction using real-time medical measurements, MeMeA Proc., IEEE International Workshop on Medical Measurements and Applications, pp 65-70.

(Gilchrist et al., 2011b) Gilchrist, J., M. Frize, C. M. Ennett, and E. Bariciak (2011, October). Performance evaluation of various storage formats for clinical data repositories. IEEE Transactions on Instrumentation and Measurement 60 (10), 32443252.

(Gilchrist, 2012) Gilchrist, J. (2012). Clinical Decision Support System using RealTime Data Analysis for a Neonatal Intensive Care Unit. PhD Thesis, Department of Systems and Computer Engineering, Carleton University, Ottawa, Canada.

(Goldenberg et al., 2008) Goldenberg, R. L., Culhane, J. F., Iams, J. D., and Romero, R. (2008). Epidemiology and causes of preterm birth. The lancet, 371(9606), 75-84.

(Gray et al., 2000) Gray, J. E., Safran, C., Davis, R. B., Pompilio-Weitzner, G., Stewart, J. E., Zaccagnini, L., and Pursley, D. (2000). Baby CareLink: using the internet and telemedicine to improve care for high-risk infants. Pediatrics, 106(6), 1318-1324. 
(Hack et al., 2002) Hack, M., Flannery, D. J., Schluchter, M., Cartar, L., Borawski, E., and Klein, N. (2002). Outcomes in young adulthood for very-low-birth-weight infants. New England Journal of Medicine, 346(3), 149-157.

(Haque et al., 2000) Haque, B. U., Belecheanu, R. A., Barson, R. J., and Pawar, K. S. (2000). Towards the application of case based reasoning to decision-making in concurrent product development (concurrent engineering). Knowledge-Based Systems, 13(2), 101-112.

(Horbar et al., 2012) Horbar, J. D., Carpenter, J. H., Badger, G. J., Kenny, M. J., Soll, R. F., Morrow, K. A., \& Buzas, J. S. (2012). Mortality and neonatal morbidity among infants 501 to 1500 grams from 2000 to 2009. Pediatrics, 129(6), 1019-1026.

(Hunter et al., 2003) Hunter, J., Ewing, G., Freer, Y., Logie, R., McCue, P., and McIntosh, N. (2003). NEONATE: Decision support in the neonatal intensive care unit-A Preliminary Report. In Artificial Intelligence in Medicine (pp. 41-45). Springer Berlin Heidelberg.

(Joseph et al., 2007) Joseph, K. S., Huang, L., Liu, S., Ananth, C. V., Allen, A. C., Sauve, R., and Kramer, M. S. (2007). Reconciling the high rates of preterm and postterm birth in the United States. Obstetrics \& Gynecology, 109(4), 813-822. 
(Kahn, 1994) Kahn Jr, C. E. (1994). Artificial intelligence in radiology: decision support systems. Radiographics, 14(4), 849-861.

(Kocis and Kocis, 2012) Kocis, K. C.and Kocis, D. J. (2012). U.S. Patent Application 13/721,010.

(Kohavari and Provost, 1998) Kohavi, R. and Provost, F. (1998). Glossary of terms. Editorial for the Special Issue on Applications of Machine Learning and the Knowledge Discovery Process, 30(2-3).

(Kopelman, 2006) Kopelman, A. E. (2006). Understanding, avoiding, and resolving end-of-life conflicts in the NICU. The Mount Sinai Journal of Medicine, New York, 73(3), 580-586.

(Kriesel 2007, 33-38) Kriesel, D. (2007). A brief introduction to neural networks. Chapter 3, pp 33-38. Available at:

http://www.dkriesel.com/en/science/neural_networks. Accessed November 20, 2014.

(Kriesel 2007b, 53-54) Kriesel, D. (2007). A brief introduction to neural networks. Chapter 4, pp 53-54. 
(Lantos and Meadow, 2011) Lantos, J. D., and Meadow, W. L. (2011). Costs and End of Life Care in the NICU: Lessons for the MICU?. The Journal of Law, Medicine \& Ethics, 39(2), 194-200.

(McHaffie et al., 2001) McHaffie, H. E., Lyon, A. J., and Hume, R. (2001). Deciding on treatment limitation for neonates: the parents' perspective. European journal of pediatrics, $160(6), 339-344$.

(MedCalc, 2014) ROC curve analysis at MedCalc. Available at: http://www.medcalc.org/manual/roc-curves.php. Accessed November 17, 2014.

Meadow, W., Lee, G., Lin, K., \& Lantos, J. (2004). Changes in mortality for extremely low birthweight infants in the 1990s: implications for treatment decisions and resource use. Pediatrics, 113(5), 1223-1229.

(Mohamed et al., 2010) Mohamed, M. A., Nada, A., and Aly, H. (2010). Day-by-day postnatal survival in very low birth weight infants. Pediatrics, 126(2), e360-e366.

(Moorman et al., 2011) Moorman, J. R., Rusin, C. E., Lee, H., Guin, L. E., Clark, M. T., Delos, J. B., and Lake, D. E. (2011, August). Predictive monitoring for early detection of subacute potentially catastrophic illnesses in critical care. In Engineering in Medicine and Biology Society, EMBC, 2011 Annual International Conference of the IEEE (pp. 5515-5518). 
(Moorman et al., 2011b) Moorman, J. R., Carlo, W. A., Kattwinkel, J., Schelonka, R. L., Porcelli, P. J., Navarrete, C. T., and Michael O’Shea, T. (2011). Mortality reduction by heart rate characteristic monitoring in very low birth weight neonates: a randomized trial. The Journal of pediatrics, 159(6), 900-906.

(Nissen, 2007) Nissen S. (2007), Large Scale Reinforcement Learning using QSARSA $(\lambda)$ and Cascading Neural Networks. Master's thesis, Department of Computer Science, University of Copenhagen, Denmark.

(Nguyen and Widrow, 1990) Nguyen, D. and Widrow, B. (1990, June). Improving the learning speed of 2-layer neural networks by choosing initial values of the adaptive weights. In Neural Networks, 1990., 1990 IJCNN International Joint Conference on (pp. 21-26).

(Ong et al., 2013) Ong, D. E., Frize, M., Gilchrist, J., Bariciak, E., and Ennett, C. M. (2013, May). Usefulness analysis of a Clinical Data Repository design. In Medical Measurements and Applications Proceedings (MeMeA), 2013 IEEE International Symposium on (pp. 86-90).

Philips S.J., Tooley G.A. (2004). The Impact of Birth Complications on Parental Decision-Making: Could Prenatal Classes Help? Available at: 
www.aare.edu.au/data/publications/2004/phi04946.pdf. Accessed December 23, 2014.

(PPADS, 2014) Physician-PArent Decision Support System, Glossary, 2014.

(REALTROMINS Inc, 2014) REALTROMINS Inc. 2014) Available at:

http://www.realtromins.com/. Accessed on November 17, 2014.

(Rubin and Chisnell 2008, 4-6) Rubin, J. and Chisnell, D. (2008), Handbook of Usability Testing :How to Plan, Design, and Conduct Effective Tests. Chapter 1, pp 4-6. John Wiley \& Sons, Inc., Hoboken, NJ, USA.

(Ryan et al., 1993) Ryan, C. A., Byrne, P., Kuhn, S., and Tyebkhan, J. (1993). No resuscitation and withdrawal of therapy in a neonatal and a pediatric intensive care unit in Canada. The Journal of pediatrics, 123(4), 534-538.

(Rybchynski, 2005) Rybchynski, D. (2005). Design of an Artificial Neural Network Research Framework to Enhance the Development of Clinical Prediction Models. Master's Thesis, University of Ottawa, Ottawa, Ontario, Canada.

Safran, C. (2003). The collaborative edge: patient empowerment for vulnerable populations. International journal of medical informatics, 69(2), 185-190. 
(Saigal and Doyle, 2008) Saigal, S. and Doyle, L. W. (2008). An overview of mortality and sequelae of preterm birth from infancy to adulthood. The Lancet, 371(9608), 261-269.

(Schwartz, 2011) Schwartz, Peter H. (2011). Decision Aids, Prevention, and the Ethics of Disclosure. Hastings Center Report 41(2), 30-39.

(Stacey et al., 2008) Stacey, D., Murray, M. A., Legare, F., Sandy, D., Menard, P., and O'Connor, A. (2008). Decision coaching to support shared decision making: a framework, evidence, and implications for nursing practice, education, and policy. Worldviews on Evidence-Based Nursing, 5(1), 25-35.

Thommandram, A., Eklund, J. M., McGregor, C., Pugh, J. E., \& James, A. G. (2014, June). A Rule-Based Temporal Analysis Method for Online Health Analytics and Its Application for Real-Time Detection of Neonatal Spells. In Big Data (BigData Congress), 2014 IEEE International Congress on (pp. 470-477).

(Townsend, 2007) Townsend D. (2007). Clinical Trial of Estimated Risk Stratification Prediction Tool. Master's thesis, School of Information Technology and Engineering Faculty of Engineering, University of Ottawa, Ottawa, Canada.

(Townsend and Frize, 2008) Townsend, D. and Frize, M. (2008, August).

Complimentary artificial neural network approaches for prediction of events in the 
neonatal intensive care unit. In Engineering in Medicine and Biology Society, 2008. EMBS 2008. 30th Annual International Conference of the IEEE (pp. 4605-4608).

(Tyson, 1995) Tyson, J. (1995). Evidence-based ethics and the care of premature infants. The future of children, 197-213.

(Watson and Marir, 1994) Watson, I. and Marir, F. (1994). Case-based reasoning: A review. The knowledge engineering review, 9(04), 327-354.

(Weiner et al., 2011) Weiner, J., Sharma, J., Lantos, J., and Kilbride, H. (2011). How infants die in the neonatal intensive care unit: trends from 1999 through 2008. Archives of pediatrics and adolescent medicine, 165(7), 630-634.

(Weyand, 2011) Weyand, S. (2011). Development and Usability Testing of a Neonatal Intensive Care Unit Physician-Parent Decision Support Tool (PPADS). Master's thesis, University of Ottawa, Ottawa, Ontario, Canada.

(Weyand et al, 2011) Weyand, S. A., Frize, M., Bariciak, E., and Dunn, S. (2011, August). Development and usability testing of a parent decision support tool for the neonatal intensive care unit. In Engineering in Medicine and Biology Society, EMBC, 2011 Annual International Conference of the IEEE (pp. 6430-6433). 
(Yee et al., 2006) Yee, W., and Ross, S. (2006). Communicating with parents of highrisk infants in neonatal intensive care. Paediatrics \& child health, 11(5), 291.

(Zweig and Campbell, 1993) Zweig, M. H. and Campbell, G. (1993). Receiveroperating characteristic (ROC) plots: a fundamental evaluation tool in clinical medicine. Clinical chemistry, 39(4), 561-577. 


\section{Appendices}

\section{Appendix A - The NICU environment}

Table A-1: Description of the types of neonates admitted to the NICU (Weyand, 2011)

\begin{tabular}{|c|c|c|}
\hline \multirow[t]{3}{*}{$\begin{array}{l}\text { Premature } \\
\text { neonates }\end{array}$} & Preterm birth (PTB) & $\begin{array}{l}\text { Born between } 35 \text { weeks of gestation } \\
\text { and } 37 \text { weeks of gestation }\end{array}$ \\
\hline & $\begin{array}{l}\text { Very preterm birth } \\
\text { (VPTB) }\end{array}$ & $\begin{array}{l}\text { Born between } 29 \text { weeks of gestation } \\
\text { and } 35 \text { weeks of gestation }\end{array}$ \\
\hline & $\begin{array}{l}\text { Extremely preterm birth } \\
\text { (EPTB }\end{array}$ & $\begin{array}{l}\text { Born born before } 28 \text { weeks of } \\
\text { gestation }\end{array}$ \\
\hline \multirow[t]{3}{*}{$\begin{array}{l}\text { Lower than normal } \\
\text { birth weight neonates }\end{array}$} & Low Birth Weight (LBW) & $\begin{array}{l}\text { Born weighing between } 1500 \mathrm{~g} \text { and } \\
2500 \mathrm{~g}\end{array}$ \\
\hline & $\begin{array}{l}\text { Very Low Birth Weight } \\
\text { (VLBW) }\end{array}$ & $\begin{array}{l}\text { Born weighing between } 1000 \mathrm{~g} \text { and } \\
1500 \mathrm{~g}\end{array}$ \\
\hline & $\begin{array}{l}\text { Extremely Low Birth } \\
\text { Weight (ELBW) }\end{array}$ & Born weighing less than $1000 \mathrm{~g}$ \\
\hline High risk neonates & \multicolumn{2}{|c|}{$\begin{array}{l}\text { Neonates with congenital abnormalities, diseases or other } \\
\text { complications }\end{array}$} \\
\hline
\end{tabular}

Table A-2: Common Medical Conditions in the NICU (Weyand, 2011), (PPADS, 2014)

\begin{tabular}{|c|c|}
\hline Apnea & $\begin{array}{l}\text { A pause in breathing lasting } 20 \text { seconds or longer. Infants usually } \\
\text { start breathing on their own, but sometimes stimulation or } \\
\text { medication is required. It is often accompanied by a slowing of } \\
\text { the heart rate known as bradycardia and the baby turning blue. }\end{array}$ \\
\hline $\begin{array}{l}\text { Bronchopulmonary } \\
\text { dysplasia (BPD) }\end{array}$ & $\begin{array}{l}\text { A chronic lung disease that is the result of injury to the lungs and } \\
\text { leads to trouble breathing and the need for extra oxygen. Also } \\
\text { known as Chronic Lung Disease (CLD). }\end{array}$ \\
\hline $\begin{array}{l}\text { Intraventricular } \\
\text { Hemorrhage (IVH) }\end{array}$ & $\begin{array}{l}\text { Bleeding in the area of the ventricles of the brain caused by the } \\
\text { bursting of fragile blood vessels. The ventricles are two small, } \\
\text { fluid filled spaces in the center of the brain. The bleeding is } \\
\text { graded on a scale of } 1-4 \text {. } \\
\text { - Grade } 1 \text { is very minor and only involves small blood clots near }\end{array}$ \\
\hline
\end{tabular}




\begin{tabular}{|c|c|}
\hline & $\begin{array}{l}\text { the edges of the ventricles. } \\
\text { - Grade } 2 \text { involves the ventricles filling with blood. } \\
\text { - Grade } 3 \text { involves the ventricles becoming enlarged and swollen } \\
\text { by the blood. } \\
\text { - Grade } 4 \text { involves bleeding within the surrounding brain tissue. } \\
\text { Babies with severe IVH (Grade } 3 \text { and Grade } 4 \text { ) have an increased } \\
\text { risk of permanent brain injury which may result in physical and } \\
\text { mental handicaps. } \\
\text { Diagnostic testing includes ultrasound of the head, computed } \\
\text { tomography (CT scan) or Magnetic Resonance Imaging (MRI). }\end{array}$ \\
\hline $\begin{array}{l}\text { Necrotizing } \\
\text { enterocolitis (NEC) }\end{array}$ & $\begin{array}{l}\text { Swelling of the intestine resulting in tissue death caused by an } \\
\text { infection or decreased blood supply. }\end{array}$ \\
\hline $\begin{array}{l}\text { Patent ductus } \\
\text { arteriosus (PDA) }\end{array}$ & $\begin{array}{l}\text { An extra communication between the lung and heart. Before birth } \\
\text { the ductus arteriosus allows the baby's blood to bypass the lungs. } \\
\text { The bypass should close shortly after birth, but if it does not it is } \\
\text { known as PDA. }\end{array}$ \\
\hline $\begin{array}{l}\text { Periventricular } \\
\text { leukomalacia (PVL) }\end{array}$ & $\begin{array}{l}\text { Periventricular tissue is located around the two fluid filled } \\
\text { ventricles in the brain. If there is not enough blood reaching the } \\
\text { periventricular tissue the tissue may die and be replaced by cysts } \\
\text { or fluid. The brain tissue that dies may affect muscle control in } \\
\text { the limbs. PVL is associated with cerebral palsy and other } \\
\text { developmental problems. }\end{array}$ \\
\hline Pneumothorax & $\begin{array}{l}\text { Involves air leaking from an injured lung into the space between } \\
\text { the lung and the chest wall. Small leaks may cause no problems } \\
\text { but large leaks may cause the lung to collapse and may require } \\
\text { drainage through a tube. }\end{array}$ \\
\hline $\begin{array}{l}\text { Respiratory Distress } \\
\text { Syndrome (RDS) }\end{array}$ & $\begin{array}{l}\text { Respiratory problem due to absence of surfactant, which is a fluid } \\
\text { produced in the mature lung that prevents the lung from becoming } \\
\text { stiff and collapsing. }\end{array}$ \\
\hline Seizures & $\begin{array}{l}\text { A "short-circuiting" of electrical activity in the brain causing } \\
\text { irregular muscle activity. Generalized seizures affect the whole } \\
\text { body. Focal seizures affect only one part or side of the body. }\end{array}$ \\
\hline Sepsis & $\begin{array}{l}\text { Infection of the blood stream involving bacteria, fungus, or a } \\
\text { virus which makes the baby very ill and affects multiple organ } \\
\text { systems. This condition is treated with antibiotics or } \\
\text { antimicrobials. }\end{array}$ \\
\hline
\end{tabular}


Table A-3: Common Medical Therapies in the NICU (Weyand, 2011), (PPADS, 2014)

\begin{tabular}{|c|c|}
\hline Artificial ventilation & $\begin{array}{l}\text { Breathing is helped by a machine called a ventilator which } \\
\text { supplies and regulates air flow and oxygen content. The air flows } \\
\text { through a tube which is inserted through the nose or mouth, down } \\
\text { the back of the throat and into the windpipe. }\end{array}$ \\
\hline Artificial nutrition & $\begin{array}{l}\text { Receiving nutrition through a tube instead of by eating. The types } \\
\text { of artificial nutrition differ based on the type of nutrition being } \\
\text { given and the location of the tube. } \\
\text { - Total parenteral nutrition (TPN): the tube is placed in a vein and } \\
\text { IV fluid nutrtition is given } \\
\text { - Nasogastic tube (NG tube): the tube is inserted in the nose and } \\
\text { delivers milk or formula to the stomach } \\
\text { - Gastrostomy tube: the tube is surgically placed directly into the } \\
\text { stomach (G tube) through the abdominal wall. }\end{array}$ \\
\hline Antibiotic therapy & $\begin{array}{l}\text { Administering antibiotics; Medication that destroys bacteria and is } \\
\text { used to treat infections. }\end{array}$ \\
\hline $\begin{array}{l}\text { Blood Product } \\
\text { Transfusions }\end{array}$ & $\begin{array}{l}\text { A treatment in which blood from a donor is administered } \\
\text { intravenously to the baby's blood. }\end{array}$ \\
\hline Catheter drainage & $\begin{array}{l}\text { A hollow flexible tube for insertion into a body cavity, duct, or } \\
\text { vessel to allow passage of fluids into and out of the body. }\end{array}$ \\
\hline Chest tube drainage & $\begin{array}{l}\text { A small plastic tube placed through the chest wall into the space } \\
\text { between the lung and the chest to remove air or fluid. }\end{array}$ \\
\hline Intravenous therapy & $\begin{array}{l}\text { Delivering fluids through a catheter in a vein that provides } \\
\text { hydration, nutrition, or medications. }\end{array}$ \\
\hline
\end{tabular}




\section{Appendix B - Result File}

\begin{tabular}{|c|c|c|c|c|c|c|c|c|c|c|}
\hline 4 & A & B & C & D & E & $\mathrm{F}$ & G & H & I & $J$ \\
\hline 1 & Weights & Conenctic & Hidden $n^{\prime}$ & - Activatior & Steepnes: & Training $\mathrm{E}$ & Training a & Learning & I Momentu & Decay-Qu \\
\hline 2 & & & & & & & & & & \\
\hline 3 & Randomis & 0.1 & 1 & 1 & 0.1 & 1 & 1 & 0.7 & 0 & -0.0001 \\
\hline 4 & Randomis & 0.1 & 1 & 1 & 0.1 & 1 & 4 & 0.7 & 0 & -0.0001 \\
\hline 5 & Randomis & 0.1 & 1 & 1 & 0.1 & 1 & 4 & 1 & 0 & -0.0001 \\
\hline 6 & Randomis & 0.1 & 1 & 1 & 0.1 & 2 & 1 & 1 & 0 & -0.0001 \\
\hline 7 & Randomis & 0.1 & 1 & 1 & 0.1 & 2 & 4 & 0.4 & 0 & -0.0001 \\
\hline 8 & Randomis & 0.1 & 1 & 1 & 0.1 & 2 & 4 & 0.7 & 0 & -0.0001 \\
\hline 9 & Randomis & 0.1 & 1 & 1 & 0.1 & 2 & 4 & 1 & 0 & -0.0001 \\
\hline 10 & Randomis & 0.1 & 1 & 1 & 0.4 & 1 & 1 & 1 & 0 & -0.0001 \\
\hline 11 & Randomis & 0.1 & 1 & 1 & 0.4 & 1 & 2 & 0.7 & 0 & -0.0001 \\
\hline 12 & Randomis & 0.1 & 1 & 1 & 0.4 & 1 & 2 & 1 & 0 & -0.0001 \\
\hline 13 & Randomis & 0.1 & 1 & 1 & 0.4 & 1 & 3 & 1 & 0 & -0.0001 \\
\hline 14 & Randomis & 0.1 & 1 & 1 & 0.4 & 1 & 4 & 0.4 & 0 & -0.0001 \\
\hline 15 & Randomis & 0.1 & 1 & 1 & 0.4 & 1 & 4 & 0.7 & 0 & -0.0001 \\
\hline 16 & Randomis & 0.1 & 1 & 1 & 0.4 & 1 & 4 & 1 & 0 & -0.0001 \\
\hline 17 & Randomis & 0.1 & 1 & 1 & 0.4 & 2 & 1 & 1 & 0 & -0.0001 \\
\hline 18 & Randomis & 0.1 & 1 & 1 & 0.4 & 2 & 2 & 0.4 & 0 & -0.0001 \\
\hline 19 & Randomis & 0.1 & 1 & 1 & 0.4 & 2 & 2 & 0.7 & 0 & -0.0001 \\
\hline 20 & Randomis & 0.1 & 1 & 1 & 0.4 & 2 & 2 & 1 & 0 & -0.0001 \\
\hline 21 & Randomis & 0.1 & 1 & 1 & 0.4 & 2 & 4 & 0.1 & 0 & -0.0001 \\
\hline 22 & Randomis & 0.1 & 1 & 1 & 0.4 & 2 & 4 & 0.4 & 0 & -0.0001 \\
\hline 23 & Randomis & 0.1 & 1 & 1 & 0.4 & 2 & 4 & 0.7 & 0 & -0.0001 \\
\hline 24 & Randomis & 0.1 & 1 & 1 & 0.4 & 2 & 4 & 1 & 0 & -0.0001 \\
\hline 25 & Randomis & 0.1 & 1 & 1 & 0.7 & 1 & 1 & 1 & 0 & -0.0001 \\
\hline 26 & Randomis & 0.1 & 1 & 1 & 0.7 & 1 & 2 & 0.4 & 0 & -0.0001 \\
\hline 27 & Randomis & 0.1 & 1 & 1 & 0.7 & 1 & 2 & 0.7 & 0 & -0.0001 \\
\hline 28 & Randomis & 0.1 & 1 & 1 & 0.7 & 1 & 2 & 1 & 0 & -0.0001 \\
\hline 29 & Randomis & 0.1 & 1 & 1 & 0.7 & 1 & 3 & 0.7 & 0 & -0.0001 \\
\hline 30 & Randomis & 0.1 & 1 & 1 & 0.7 & 1 & 4 & 0.1 & 0 & -0.0001 \\
\hline 31 & Randomis & 0.1 & 1 & 1 & 0.7 & 1 & 4 & 0.7 & 0 & -0.0001 \\
\hline 32 & Randomis & 0.1 & 1 & 1 & 0.7 & 1 & 4 & 1 & 0 & -0.0001 \\
\hline 33 & Randomis & 0.1 & 1 & 1 & 0.7 & 2 & 1 & 1 & 0 & -0.0001 \\
\hline 34 & Randomis & 0.1 & 1 & 1 & 0.7 & 2 & 2 & 0.1 & 0 & -0.0001 \\
\hline 35 & Randomis & 0.1 & 1 & 1 & 0.7 & 2 & 2 & 0.4 & 0 & -0.0001 \\
\hline 36 & Randomis & 0.1 & 1 & 1 & 0.7 & 2 & 2 & 0.7 & 0 & -0.0001 \\
\hline 37 & Randomis & 0.1 & 1 & 1 & 0.7 & 2 & 2 & 1 & 0 & -0.0001 \\
\hline 38 & Randomis & 0.1 & 1 & 1 & 0.7 & 2 & 4 & 0.1 & 0 & -0.0001 \\
\hline
\end{tabular}

Figure B-1: The first half of the result file created by the MIRG ANN Builder 


\begin{tabular}{|c|c|c|c|c|c|c|c|c|}
\hline K & L & M & $\mathrm{N}$ & 0 & $\mathrm{P}$ & Q & $\mathrm{R}$ & $\mathrm{S}$ \\
\hline Mufactor- & Step_init- & Step_inc- 1 & ep_dec- & -Test_Sn & Test_Sp & Ver_Sn & Ver_Sp & File name ar \\
\hline 1.75 & 0.1 & 1.2 & 0.5 & 75 & 96.6667 & 75 & 96.6667 & 7 ../Networks/ \\
\hline 1.75 & 0.1 & 1.2 & 0.5 & 75 & 98 & 62.5 & 99.3333 & ../Networks/ \\
\hline 1.75 & 0.1 & 1.2 & 0.5 & 75 & 98 & 62.5 & 99.3333 & .../Networks/ \\
\hline 1.75 & 0.1 & 1.2 & 0.5 & 75 & 98 & 62.5 & 99.3333 & ../Networks/ \\
\hline 1.75 & 0.1 & 1.2 & 0.5 & 75 & 99.3333 & 62.5 & 99.3333 & .../Networks/ \\
\hline 1.75 & 0.1 & 1.2 & 0.5 & 75 & 99.3333 & 62.5 & 99.3333 & ../Networks/ \\
\hline 1.75 & 0.1 & 1.2 & 0.5 & 75 & 99.3333 & 62.5 & 99.3333 & ../Networks/ \\
\hline 1.75 & 0.1 & 1.2 & 0.5 & 75 & 96.6667 & 75 & 96.6667 & ../Networks/ \\
\hline 1.75 & 0.1 & 1.2 & 0.5 & 37.5 & 99.3333 & 37.5 & 100 & ../Networks/ \\
\hline 1.75 & 0.1 & 1.2 & 0.5 & 75 & 98 & 62.5 & 99.3333 & ../Networks/ \\
\hline 1.75 & 0.1 & 1.2 & 0.5 & 87.5 & 64 & 100 & 66.6667 & $7 . . /$ Networks/ \\
\hline 1.75 & 0.1 & 1.2 & 0.5 & 75 & 98 & 62.5 & 99.3333 & ../Networks/ \\
\hline 1.75 & 0.1 & 1.2 & 0.5 & 75 & 98 & 62.5 & 99.3333 & .../Networks/ \\
\hline 1.75 & 0.1 & 1.2 & 0.5 & 75 & 98 & 62.5 & 99.3333 & .../Networks/ \\
\hline 1.75 & 0.1 & 1.2 & 0.5 & 75 & 98 & 62.5 & 99.3333 & ../Networks/ \\
\hline 1.75 & 0.1 & 1.2 & 0.5 & 75 & 98 & 62.5 & 99.3333 & .../Networks/ \\
\hline 1.75 & 0.1 & 1.2 & 0.5 & 75 & 98 & 62.5 & 99.3333 & ../Networks/ \\
\hline 1.75 & 0.1 & 1.2 & 0.5 & 75 & 98 & 62.5 & 99.3333 & .../Networks/ \\
\hline 1.75 & 0.1 & 1.2 & 0.5 & 75 & 98 & 62.5 & 99.3333 & ../Networks/ \\
\hline 1.75 & 0.1 & 1.2 & 0.5 & 75 & 98 & 62.5 & 99.3333 & ../Networks/ \\
\hline 1.75 & 0.1 & 1.2 & 0.5 & 75 & 98 & 62.5 & 99.3333 & ../Networks/ \\
\hline 1.75 & 0.1 & 1.2 & 0.5 & 75 & 98 & 62.5 & 99.3333 & ../Networks/ \\
\hline 1.75 & 0.1 & 1.2 & 0.5 & 75 & 96.6667 & 75 & 96.6667 & ../Networks/ \\
\hline 1.75 & 0.1 & 1.2 & 0.5 & 75 & 98 & 62.5 & 99.3333 & ../Networks/ \\
\hline 1.75 & 0.1 & 1.2 & 0.5 & 75 & 98 & 62.5 & 99.3333 & ../Networks/ \\
\hline 1.75 & 0.1 & 1.2 & 0.5 & 75 & 98 & 62.5 & 99.3333 & .../Networks/ \\
\hline 1.75 & 0.1 & 1.2 & 0.5 & 87.5 & 67.3333 & 87.5 & 74 & .../Networks/ \\
\hline 1.75 & 0.1 & 1.2 & 0.5 & 75 & 98 & 62.5 & 99.3333 & ../Networks/ \\
\hline 1.75 & 0.1 & 1.2 & 0.5 & 75 & 98 & 62.5 & 99.3333 & ../Networks/ \\
\hline 1.75 & 0.1 & 1.2 & 0.5 & 75 & 98 & 62.5 & 99.3333 & .../Networks/ \\
\hline 1.75 & 0.1 & 1.2 & 0.5 & 75 & 98 & 62.5 & 99.3333 & ../Networks/ \\
\hline 1.75 & 0.1 & 1.2 & 0.5 & 12.5 & 100 & 25 & 100 & ../Networks/ \\
\hline 1.75 & 0.1 & 1.2 & 0.5 & 75 & 98 & 62.5 & 99.3333 & ../Networks/ \\
\hline 1.75 & 0.1 & 1.2 & 0.5 & 75 & 98 & 62.5 & 99.3333 & ../Networks/ \\
\hline 1.75 & 0.1 & 1.2 & 0.5 & 75 & 98 & 62.5 & 99.3333 & ../Networks/ \\
\hline 1.75 & 0.1 & 1.2 & 0.5 & 75 & 98 & 62.5 & 99.3333 & ../Networks/ \\
\hline
\end{tabular}

Figure B-2: The second half of the result file created by the MIRG ANN Builder 


\section{Appendix C - Ethics clearance}

\section{Carleton \\ Canada's Capital University}

Carleton University Research Office

Research Ethics Board

1325 Dunton Tower

1125 Colonel By Drive

Ottawa, ON K1S 5B6 Canada

Tel: $613-520-2517$

ethics@icarleton.ca

\section{Ethics Clearance Form}

This is to certify that the Carleton University Research Ethics Board has examined the application for ethical clearance. The REB found the research project to meet appropriate ethical standards as outlined in the Tri-Council Policy Statement: Ethical Conduct for Research Involving Humans, $2^{\text {nd }}$ edition and, the Carleton University Policies and Procedures for the Ethical Conduct of Research.

X New clearance

- Renewal of original clearance Original date of clearance:

Date of clearance

Researcher

Department

Supervisor

Project number

Title of project

\author{
31 January 2014 \\ Hasmik Martirosyan, Master's students \\ Systems and Computer Engineering \\ Prof. Monique Frize, Systems and Computer Engineering \\ 100556 \\ Study of Neonatal Intensive Care Unit physician-parent decision \\ support tool
}

Clearance expires: 31 May 2014

All researchers are governed by the following conditions:

Annual Status Report: You are required to submit an Annual Status Report to either renew clearance or close the file. Failure to submit the Annual Status Report will result in the immediate suspension of the project. Funded projects will have accounts suspended until the report is submitted and approved.

Changes to the project: Any changes to the project must be submitted to the Carleton University Research Ethics Board for approval. All changes must be approved prior to the continuance of the research.

Adverse events: Should any participant suffer adversely from their participation in the project you are required to report the matter to the Carleton University Research Ethics Board. You must submit a written record of the event and indicate what steps you have taken to resolve the situation.

Suspension or termination of clearance: Failure to conduct the research in accordance with the principles of the Tri-Council Policy Statement: Ethical Conduct for Research Involving Humans, $2^{\text {nd }}$ edition and the Carleton University Policies and Procedures for the Ethical Conduct of Research may result in the suspension or termination of the research project.

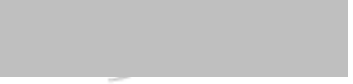

Andy Adler, Chair Carleton University Research Ethics Board

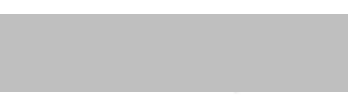

Louise Heslop, Vice-Chair Carleton University Research Ethics Board 


\section{Ethics Clearance Form - Clearance Renewal}

This is to certify that the Carleton University Research Ethics Board has examined the application for ethical clearance. The REB found the research project to meet appropriate ethical standards as outlined in the Tri-Council Policy Statement: Ethical Conduct for Research Involving Human, 2nd edition, and the Carleton University Policies and Procedures for the Ethical Conduct of Research.

Original Date of Clearance: January 31, 2014

Renewal Date of Clearance: May 24, 2014

Researcher: Hasmik Martirosyan (Student Research: Master's Student)

Department: Faculty of Engineering and DesignISystems and Computer Engineering (Department of)

University: Carleton University

Research Supervisor (if applicable): Monique Frize

Project Number: 100556

Alternate File Number (if applicable):

Project Title: Study of Neonatal Intensive Care Unit physician-parent decision support tool

Clearance Expires: 31 May 2015

All researchers are governed by the following conditions:

Annual Status Report: You are required to submit an Annual Status Report to either renew clearance or close the file. Failure to submit the Annual Status Report will result in the immediate suspension of the project. Funded projects will have accounts suspended until the report is submitted and approved.

Changes to the project: Any changes to the project must be submitted to the Carleton University Research Ethics Board for approval. All changes must be approved prior to the continuance of the research.

Adverse events: Should a participant suffer adversely from their participation in the project you are required to report the matter to the Carleton University Research Ethics Board. You must submit a written record of the event and indicate what steps you have taken to resolve the situation.

Suspension or termination of clearance: Failure to conduct the research in accordance with the principles of the Tri-Council Policy Statement: Ethical Conduct for Research Involving Humans, 2nd edition and the Carleton University Policies and Procedures for the Ethical Conduct of Research may result in the suspension or termination of the research project.

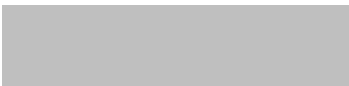

Andy Adler, Chair

Carleton University Research Ethics Board

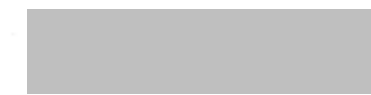

Louise Heslop, Vice-Chair

Carleton University Research Ethics Board 
Research Ethics Board

2014 Annual Renewal (Delegated)

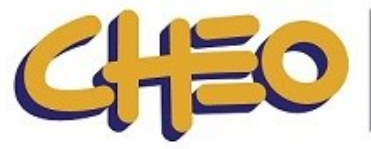

RESEARCH INSTITUTE

INSTITUT DE RECHERCHE

Principal Investigator: Dr. Erika Bariciak

REB Protocol No: 10/144X

Romeo File No: 10001398

Project Title: Study of a Neonatal Intensive Care Unit Parent Decision Support Tool (PPADS)

Primary Affiliation: Clinical Research\Neonatology

Contingencies: None

Protocol Status: Active

Approval Date: January 14, 2014

Approval Valid Until: January 15, 2015

Annual Renewal Submission Deadline: December 15, 2014

Documents Reviewed \& Approved:

\begin{tabular}{|l|l|l|}
\hline Document Name & Comments & Version Date \\
\hline Other Document & Signed Renewal Form & $2013 / 12 / 19$ \\
\hline Protocol & Current Protocol & $2013 / 11 / 14$ \\
\hline
\end{tabular}

This is to notify you that the CHEO REB has granted approval to the renewal for the above named research study for a period of one year. The renewal was reviewed and approved by the Chair only. Decisions made by the Chair under delegated review are ratified by the full Board at its subsequent meeting.

In fulfilling its mandate, the CHEO REB is guided by: Tri-Council Policy Statement; ICH Good Clinical Practice Practices: Consolidated Guideline; Applicable laws and regulations of Ontario and Canada (e.g., Health Canada Division 5 of the Food and Drug Regulations $\&$ the Food and Drugs Act - Medical Devices Regulations).

Approval is granted with the understanding that the investigator agrees to comply with the following requirements:

1. The investigator must conduct the study in compliance with the protocol and any additional conditions set out by the Board.

2. The investigator must not implement any deviation from, or changes to, the protocol without the approval of the REB except where necessary to eliminate an immediate hazard to the research subject, or when the change involves only logistical or administrative aspects of the study (e.g., change of telephone number 
or research staff). As soon as possible, however, the implemented deviation or change, the reasons for it, and, if appropriate, the proposed protocol amendment(s) should be submitted to the Board for review.

3. The investigator must, prior to use, submit to the Board changes to the study documentation, e.g., changes to the informed consent letters, recruitment materials. Should major revisions to the consent form be made, the investigator agrees to re-consent those subjects who have originally consented to the study and who wish to continue on the study.

4. For clinical drug or device trials, investigators must promptly report to the REB all adverse events that are both serious and unexpected (SAEs). For SAE reports on CHEO patients, the investigator must also comply with the hospital-wide Policy regarding, Procedures For Considering Medical Error In The Differential Diagnosis of Severe Adverse Events (SAE) Associated with the Drugs Administered in a Clinical Trial (see

5. For all other research studies, investigators must promptly report to the REB all unexpected and untoward occurrences (including the loss or theft of study data and other such privacy breaches).

6. Investigators must promptly report to the REB any new information regarding the safety of research subjects (e.g., changes to the product monograph or investigator's brochure for drug trials). Where available, any reports produced by Data Safety Monitoring Board should be submitted to the REB.

7. Investigators must notify the REB of any study closures (temporary, premature or permanent), in writing along with an explanation of the rationale for such action.

8. Investigators must submit an annual renewal report to the REB 30 days prior to the expiration date stated on the final approval letter.

9. Investigators must submit a final report at the conclusion of the study.

10. Investigators must provide the Board with French version of the consent form, unless a waiver has been granted.

If you have any questions, pertaining to this letter, please contact Natalie Anderson, Research Ethics Board office at ( or

Regards,

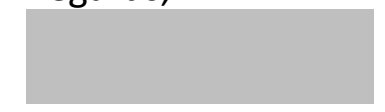

Dr. Carole Gentile

Chair, Research Ethics Board

Présidente, Comité d'éthique de la recherche

401 Smyth Road, Ottawa, ON K1H 8L1 


\section{Appendix D Pilot Study Documents}

\section{D.1 Recruitment Letter}

Re: Study of a Neonatal Intensive Care Unit Parent-Physician Decision Support Tool To,

We are contacting you to ask if you would be interested in participating in a study to determine the usefulness and ease of use of a tool we have designed to help parents make difficult decisions about their sick baby in to the NICU. The study will be conducted at the Children's Hospital of Eastern Ontario (CHEO) and has been approved by the CHEO Research Ethics Board.

As a parent who has unfortunately experienced the loss of an infant in the NICU, we understand that this study may be emotionally difficult for you. As someone who had to make very difficult decisions about your baby, you are able to help us understand what the needs may be for other parents experiencing the same situation. This will help us to ensure our tool is doing the very best to help parents and families understand their baby's medical condition and the different treatment options that the medical team may recommend.

Hasmik Martirosyan is a master's student at Carleton University and will be conducting the study under the supervision of Dr. Erika Bariciak who is a staff Neonatologist at CHEO. She is investigating the development of a parent-physician decision support tool known as PPADS. The PPADS tool is a computer-based, interactive decision aid to be used by parents with very ill neonates in the neonatal intensive care unit.

Participation will involve a 30-45 minute session at CHEO. We will ask you to complete a short questionnaire providing us with some background information about yourself. We will then present you with a simulated case of a preterm infant who is very sick and not responding to therapy. Afterwards, we will ask you to use the PPADS tool to help you understand the baby's condition and what treatment options are available. We will then ask you to complete a second short questionnaire telling us what you thought about the tool. Although participating in this study would not have any direct benefit to 
you, it may benefit parents with very ill neonates in the future by providing us with feedback that will help us to ensure that the tool best addresses the needs of parents who are making difficult decisions about their sick baby.

As we will be describing a very ill neonate, we recognize that this may cause you emotional distress. Should you need further support, we can arrange for you to meet with our NICU Social Worker. There is no payment for your participation in the study, but your parking pass at CHEO will be paid. You will not be identified in any published materials related to this study.

Participation in this research study is voluntary and is very much appreciated. Please indicate your interest and return this letter in the prepaid envelope provided by date to be determined.

$\square$ I am interested. Please have the NICU research assistant contact me at the following email or phone number.

Email:

Phone Number:

$\square$ I am interested and will contact the NICU research assistant (Samantha Somers) at .

Sincerely,

Dr. Thierry Lacaze

Chief of Neonatology

Children's Hospital of Eastern Ontario 


\section{D.2 Study Test Participant Script}

Title of research project: Study of a Neonatal Intensive Care Unit Parent-Physician

\section{Decision Support Tool}

Participant ID:

"Thank you for agreeing to take part in our research study. My name is Hasmik Martirosyan and I am a research student from Carleton University.

During the rest of the session, I will be working from a script to ensure that my instructions are the same for everyone participating in this study.

My master's research explores the development, usefulness and ease of use of a decision support tool for parents who have a very ill neonate in the neonatal intensive care unit at CHEO. This study is designed to determine the usefulness of the tool, how easy it is to use, and the effectiveness of our physician-parent decision support tool which we have named "PPADS".

During the session, I will ask you to use our current design of the decision support tool to do a variety of tasks and I will observe you while you do them. As you complete the tasks, please try to do whatever you would normally do. Please try to think and read out loud while you're working, as this will provide more information about the usability of the tool. Please know that we're not evaluating you, and there are no wrong answers. I encourage you to ask questions at any time. You will also be asked to complete a background questionnaire and an evaluation questionnaire. All questionnaires and notes will be tracked by a random number which has been assigned to you. You will not be identified in any data analysis or publication. The whole session will take between 30 and 45 minutes.

Some of the information you will be presented with today includes two simulated cases about a sick baby in the NICU. Although we know that in reality the NICU experience and ongoing discussions with the medical team provide parents with a more detailed understanding of their baby's condition, the use of simulated cases of this type will still give us a sense of how parents might approach decision making in the NICU. We understand that this also might be upsetting for you to think about. I will provide you with the contact information of our Social Worker before you leave, in case you would like to have some follow-up support. Do you have any questions before we begin?

Please take a moment to read and complete this consent form. At this point I would like to remind you that you may withdraw from this study at any point. [Wait] Thank you.

Next, could you please complete the Background Questionnaire? [Wait] Thank you. 
Next, I would like to ask you to please complete the three tasks on this sheet. I ask that you please perform one task at a time, and do not skip any tasks. Please let me know when you have finished. [Wait] Thank you.

Finally, I ask that you fill out the evaluation questionnaire. [Wait] Thank you very much for your participation in this research study." 


\section{D.3 Information Sheet and Parent Consent Form}

Title of research project: Study of a Neonatal Intensive Care Unit Physician-Parent Decision Support Tool

Principal Investigator: Dr. Erika Bariciak

Participant ID number:

\section{Introduction}

You are being asked to participate in this research project to determine the usefulness and ease of use of a parent decision support tool known as: Physician-Parent Decision Support (PPADS). The PPADS tool is a computer-based decision support tool to be used by parents of very ill neonates admitted to the Neonatal Intensive Care Unit (NICU) who face difficult care decisions.

Please read this Patient Information Sheet and Consent Form carefully and ask as many questions as you like before deciding whether to participate in the research study. You can discuss this decision with your family, friends and your healthcare team.

\section{Background, Purpose and Design of the Study}

The purpose of this study is to better understand what parents are looking for when using a decision support tool. There are many things to consider when thinking about your infants' care. This tool is meant to be used to help parents by providing them with information on their baby's condition, what options there are for their baby's care, and what the possible outcomes might be, depending on the decisions made.

\section{Study Procedures}

You will be asked to complete a background questionnaire. The background questionnaire will ask general questions about your gender, age, education level, and NICU experience. After the background questionnaire is complete, we would like you to use the decision support tool for about 20 minutes, performing three specific tasks related to support for parental decisions in the NICU. Each task will present you with a fictitious scenario, where a very ill neonate admitted to the NICU requires difficult decisions regarding their care. While completing these three tasks, you will be asked to try to think out loud, describing what you are thinking and doing. After completing the three specific tasks, you will then be asked to complete an evaluation questionnaire to evaluate the tool 
and tell us your general impressions of the tool. The study is not evaluating you; in fact, if you have trouble using the tool, this information will be very helpful for improving the design of the tool to suit parents of babies admitted to the NICU.

\section{Study Duration}

Participation will involve one 30-45-minute session at the Children's Hospital of Eastern Ontario (CHEO), near the Neonatal Intensive Care Unit (NICU).

\section{Possible Risks}

As you have had an experience in the NICU with a sick baby in the past, there is potential for emotional or psychological distress. We encourage you to keep an open communication about how you are feeling throughout the study. If, at any point, you wish to take a short break or stop your participation in the study, please feel free to do so. We will provide you with the contact information of our NICU Social Worker upon completion of the study, in case you would like to arrange a consult with her for support.

\section{Benefits of the Study}

Participating in this study may have no direct benefit to you, but may help parents with very ill neonates admitted to the NICU make more informed choices for their babies in the future. It will also help us to ensure the tool best addresses the needs of parents making difficult care decisions about their sick baby.

\section{Withdrawal from the Study}

You have the right to withdraw from the study at any time. If you decide to withdraw, you may discuss this with the study assistant before you stop the study, or contact her at any time after completion of the study.

If you withdraw your consent, the study investigators will no longer use and disclose any information under the consent for this study.

\section{Study Costs}

You will not be paid to participate in this research study, but you will be reimbursed for parking directly related to this study.

\section{Confidentiality}


All personal health information will be kept confidential, unless release is required by law. Representatives from the CHEO Research Ethics Board, as well as the CHEO Research Institute, may access this information under the supervision of Dr. Erika Bariciak for audit purposes.

You will not be identifiable in any publications or presentations resulting from this study. No identifying information will leave CHEO. All information which leaves the hospital will be coded with an independent study number.

The link between your name and the independent study number will only be accessible by Dr. Erika Bariciak and her staff. The link and study files will be stored separately and securely. Both files will be kept for a period of 15 years after the study has been completed. All paper records will be stored in a locked file and office. All electronic records will be stored on the CHEO server and protected by a user password, again only accessible by Dr. Erika Bariciak and her staff. At the end of the retention period, all paper records will be disposed of in confidential waste or shredded, and all electronic records will be deleted.

\section{Voluntary Participation}

Your participation in this study is voluntary. If you choose not to participate, your decision will not affect the care you or your child receives at this Institution in the future. You will not have any penalty or loss of benefits to which you are otherwise entitled to.

\section{Questions about the Study}

If you have any questions about the study, please contact Dr. Erika Bariciak at

The Children's Hospital of Eastern Ontario Research Ethics Board (CHEO REB) has reviewed and approved this research project. The CHEO REB is a committee of the hospital that includes individuals from different professional backgrounds. The REB reviews all research that takes place at the hospital. Its goal is to ensure the protection of the rights and welfare of people participating in research. You may contact the Chair of the CHEO REB, for information regarding your rights as a research subject at 


\section{Consent Form}

Study of a Neonatal Intensive Care Unit Physician-Parent Decision Support Tool (PPADS)

\section{Consent to Participate in Research}

I understand that I am being asked to participate in a research study about the usability of a Physician-Parent Decision Support Tool in the Neonatal Intensive Care Unit (NICU). This study has been explained to me by

I have read this 4 page Patient Information Sheet and Consent Form (or I have had this document read to me). All my questions have been answered to my satisfaction. If I decide at a later stage in the study that I would like to withdraw my consent, I may do so at any time.

I voluntarily agree to participate in this study.

A copy of the signed Information Sheet and/or Consent Form will be provided to me.

\section{Signatures}

Participant's Name (Please Print)

Participant's Signature

Date

\section{Investigator Statement (or Person Explaining the Consent)}

I have carefully explained to the research participant the nature of the above research study. To the best of my knowledge, the research participant signing this consent form understands the nature, demands, risks and benefits involved in participating in this study. I acknowledge my responsibility for the care and well-being of the above research participant, to respect the rights and wishes of the research participant, and to conduct the study according to applicable Good Clinical Practice guidelines and regulations.

Name of Investigator/Delegate (Please Print)

Signature of Investigator/Delegate

Date 


\section{D.4 Feuillet de Consentement Parental}

Titre du projet de recherche: Étude d'un système d'aide à la décision pour les parentsmedecins de nouveaux-nés admis aux soins intensifs

Numéro de Participant:

\section{Pourquoi cette étude?}

Cette étude a pour but de déterminer l'utilité et la facilité d'usage d'un système de support de décision pour les parents et les medecins de nouveaux-nés admis aux soins intensifs (PPADS). PPADS utilise un ordinateur et un programme pour aider les parents à prendre des décisions difficiles au sujet des soins d'un nouveau-né très malade à l'unité de soins intensifs.

\section{Qu'implique cette étude?}

L'étude va devoir recruiter un minimum de douze personnes et une session va durer de 30 à 45-minutes. Vous devrez premièrement compléter un questionnaire de base qui va poser des questions sur votre sexe, age, niveau d'éducation et votre experience au sein de l'unité de soins intensifs néonataux. Puis on vous demandera d'utiliser le système d'aide à la décision pour une vingtaine de minutes, avec des tâches spécifiques. Durant cet exercice, on vous demandera d'essayer de faire vos commentaries de vive-voix, décrivant ce que vous pensez et faites. Après avoir complété ces tâches, on vous demandera de compléter un questionnaire pour évaluer l'outil et pour identifier des problèmes. Le questionnaire pour évaluer l'outil vous demandera si l'outil est facile à utiliser, et si vous le trouvez utile. Cette étude ne vous évalue pas; en effet, si vous avez des difficultés à utiliser l'outil, ces renseignements seront très utiles pour que nous apportions des améliorations au système. Il n'y aura pas compensation pour votre participation à cette étude, mais nous payerons votre stationnement.

\section{Quels sont les risques et les bénéfices?}

Une participation à cette étude n'aura pas de bénéfices directement pour vous, mais elle pourra aider d'autres parents dans l'avenir. Il est possible qu'il y ait des effets mineurs émotifs ou psychologiques. Nous vous encourageons à communiquer vos sentiments durant toute la session. Si vous désirez faire une pause ou arrêter complètement, sentez vous libre de le faire. Vous pouvez vous retirer de l'étude à n'importe quel moment, jusqu'à une semaine après la fin de la session, et toutes les réponses et informations que vous avez fournies seront détruites.

Est ce que je serai identifié? 
La collecte de données se compose de deux questionnaires et de notes prises durant la session. Vous ne serez pas identifié dans les publications. Seul le personnel de recherche auront accès aux renseignements qui pourraient permettre de vous identifier. Toutes les données qui contiennent votre nom vont être entreposées dans un bureau, sous clef, à l'Université Carleton. 


\section{Si je decide de retirer ma participation, que dois-je faire?}

Vous êtes libre de vous retirer de l'étude à n'importe quel moment, jusqu'à une semaine après la fin de la session, et il n'y aura aucune consequence pour vous. Si vous décidez de vous retirer du projet, nous paierons votre stationnement. Nous vous prions de nous informer si vous voulez vous retirer durant la session, et si vous décidez de vous retirer à un autre moment, après la session, vous pouvez contacter Dr. Erika Bariciak au numéro suivant $\quad t$ laisser un message dans la boîte vocale confidentielle.

\section{Consentement}

En signant ce formulaire, je reconnais que je me suis porté volontaire pour participer à cette étude, pour aider à déterminer l'utilité et la facilité d'usage d'un nouvel outil. Je reconnais qu'on m'a bien expliqué la procedure à suivre, j'en ai reçu une copie, et on a répondu à toutes mes questions de manière satisfaisante. De plus, on m'a donné suffisamment de temps pour réfléchir à ma décision de participer.

On m'a assuré que je ne serai pas identifié dans les publications. Je comprends que je peux me retirer de cette étude à n'importe quel moment, jusqu'à une semaine après la fin de la session, et qu'il n'y aura aucune consequence pour moi. Toute information personnelle va être codée, donc je ne serai pas identifié par mon nom.

Un grand merci pour votre participation!

Signature du participant: Date:

Nom du participant (Imprimer):

Signature du témoin:

\section{J'AI EXPLIQUÉ CETTE ÉTUDE À LA PERSONNE QUI A SIGNÉ CI-HAUT ET JE SUIS SATISFAITE QU'ELLE EST BIEN COMPRISE.}

Signature du chercheur ou personne désignée:

Si vous avez une préoccupation au sujet de cette étude, veuillez contacter: Dr. Erika Bariciak par courriel à ] ou par téléphone à

Le CHEO Research Ethics Board (REB) a reçu et approuvé ce projet de recherche. Vous pouvez contacter Dr. Carole Gentile, présidente du Research Ethics Board, pour des renseignements au sujet des droits des participants dans un projet de recherche: ( 


\section{D.5 Parental Background Questionnaire for the PPADS Tool}

Title of research project: Study of a Neonatal Intensive Care Unit Physician-Parent Decision Support Tool

Participant ID:

The purpose of this survey is to determine some background information that will be used for the data analysis. The questionnaire should take about 5 minutes. Please be aware that you are under no obligation to complete this questionnaire, and you may withdraw from the study at any time.

1. Your age range:

$\square$ Under 29

$\square 30$ to 39

$\square 40$ to 49

$\square 50$ to 59

$\square 60$ and older

2. Your gender:

$\square$ Female

$\square$ Male

3. Is another member of your family also participating in this study?

$\square$ Yes
$\square$ No

4. Your highest level of education:

$\square$ Less than High school diploma

$\square$ High school diploma

$\square$ Some university or college years

$\square$ University degree

$\square$ Other, specify

5. How many of your children have been admitted to the NICU: 


$\square 0$
$\square 1$
$\square 2$
$\square 3$

$\square$ Other, specify

6. Length of time elapsed since your infant was in the NICU:

$\square<3$ months

$\square$ 4-6 months

$\square$ 7-9 months

$\square$ 10-12 months

$\square>12$ months

7. Were any of the following scenarios presented to you while your neonate was in the NICU:

$\square$ Withdrawal of care

$\square$ Non-escalation of care

$\square$ Do not resuscitate order

$\square$ None of the above

8. Were any of these scenarios undertaken:

$\square$ Withdrawal of care

$\square$ Non-escalation of care

$\square$ Do not resuscitate order

$\square$ None of the above

9. Do you have a computer at home?

$\square$ Yes

$\square$ No

10. How often do you use a computer per week?

$\square$ Never

$\square$ Rarely

$\square$ Every once in a while

$\square$ Often

11. What is your computer skill level?

$\square$ Novice 


$\square$ Beginner
$\square$ Advanced
$\square$ Expert

Thank you for your participation. You may provide any additional comments below: 


\section{D.6 Parental Evaluation Tasks for the PPADS Tool}

Title of research project: Study of a Neonatal Intensive Care Unit Physician-Parent

\section{Decision Support Tool}

Participant ID:

Thank you for taking the time to help us evaluate our physician-parent decision support tool (PPADS). This tool has been developed to help parents who have very ill infants in the NICU. This tool is intended to be used in addition to parent-physician meetings, to help parents better understand information about their baby and help them make difficult care decisions with their doctor.

During the evaluation, we ask you to provide your opinion of the tool and perform a few short simple tasks to evaluate it. We ask that you think out loud as you interact with the tool to provide us with more information. Feel free to ask questions at any time. You are not obliged to complete this study and you may withdraw at any time.

Please complete each of the tasks below. You may use the checkboxes to keep track of the tasks you have completed. The tasks should take about 30 minutes to complete.

\section{Task 1:}

Imagine that you are the parent of a baby girl, Jane, born 2 weeks ago at 24 weeks gestation (3 months early).

She needed help to breathe right at birth and was placed on a breathing machine (ventilator). She has very immature, injured lungs, and each attempt to remove her from the ventilator has failed.

She now has bleeding into her lungs from a problem with her heart called a PDA; this has made her lungs even sicker. She is now on the maximum level of ventilator support but is still not doing well.

She has needed blood transfusions for the lung bleeding. Her blood pressure is low and she needs medication to help keep it normal.

She has stopped voiding (producing urine) because her kidneys are failing. Her salts in her blood are severely unbalanced putting her at risk for heart rhythm problems.

An x-ray of her intestines has shown that she has a severe gut infection called NEC. She has been started on antibiotics for this.

The doctor looking after Baby Jane has met with you to discuss her situation. The medical team feels that Jane will likely not survive, despite ongoing therapy. They feel that even if she did survive, she would most likely suffer from severe handicaps including 
cerebral palsy $(\mathrm{CP})$ and intellectual deficits. They recommend changing her direction of care to palliative care and withdrawing all painful, invasive, life support.

Please use this PPADS tool to help you understand your options when considering this decision.

Steps:

1) $\square$ Log in using:

User name: 1234567

Password: 123

2) $\square$ View the home page

3) View medical information

$\square$ View the current conditions

$\square$ View the note from the clinician

$\square$ View the current treatments

$\square$ Find and read the definition of at least two of the current treatments

$\square$ View the medical outcome predictions (Risk of mortality, Risk of BPD, Risk of IVH)

4) Go to the decision support page

$\square$ View the different direction of care options

$\square$ View the reason a change in direction of care was suggested

5) Complete a new decision support form

$\square$ Fill out the decision support form (assume information when not known)

6) $\square$ Print the decision support form

7) $\square$ Go to homepage

8) $\square$ Log out

\section{Task 2:}

Imagine that you are the parent of a baby boy, Bob, who was born at term gestation 1 week ago.

He did fine for the first few days of life, but then became pale and stopped feeding. He quickly got sicker and had trouble breathing and had to be placed on a ventilator. He is still on the ventilator.

He developed low blood pressure and needed medications to keep his blood pressure normal. He continued to get worse and he developed heart and kidney failure. An echocardiogram (ECHO) was done, which showed that he has congenital heart disease, which requires open-heart surgery. A medication was started to help his heart pump blood more easily throughout his body, and this stabilized him.

His kidneys remain damaged and he is still not voiding (producing urine). This has resulted in a severe imbalance of the salts in his blood. 
An MRI of his head showed that his brain did not receive enough blood flow or oxygen and now he has widespread injury to the brain.

The doctor looking after Baby Bob would like to meet with you to discuss his current clinical status. He is worried that Baby Bob has had some irreversible injuries to many of his organs as a result of being so sick and worries about whether Bob will be able to tolerate the surgery.

Please use this PPADS tool to help you understand Bob's current issues to help you prepare for a meeting with the medical team.

Steps:

1) $\square$ Log in using:

User name: 7654321

Password: 765

2) $\square$ View home page

3) View medical information

$\square$ View the current conditions

Find and read the definition of at least two of the current conditions

$\checkmark$ View the note from the clinician

$\square$ View the current treatments

$\square$ View the medical outcome predictions (Risk of mortality, Risk of BPD, Risk of IVH)

4) $\square$ View the glossary

5) $\square$ Search glossary for "IVH"

6) $\square$ Log out

\section{Task 3:}

For this task you may log back in to either Baby Jane's or Baby Bob's account and explore the tool at your leisure.

Steps:

1) $\square$ Log in using:

User name for Jane: 1234567

Password: 123

User name for Bob: 7654321

Password: 765

2) $\square$ Explore the tool at your leisure

3) $\square$ Log out 


\section{D.7 Parental Evaluation Questionnaire for the PPADS Tool}

Title of research project: Study of a Neonatal Intensive Care Unit Physician-Parent Decision Support Tool

Participant ID:

The purpose of this study is to determine whether the PPADS tool is easy to use, useful, and has the potential to be used by parents while their baby is admitted in the neonatal intensive care unit. The questionnaire will assess your opinion about the tool and should take about 10 minutes to complete. Please be aware that you are under no obligation to complete this questionnaire and you may withdraw from the study at any time.

1. Your overall view of PPADS:

$\square$ Exceptional

$\square$ Satisfying

$\square$ Frustrating

$\square$ Unacceptable

2. The organization of information in PPADS is:

$\square$ Very clear

$\square$ Moderately clear

$\square$ Somewhat confusing

$\square$ Very confusing

3. Navigation through PPADS is:

$\square$ Very easy

$\square$ Moderately easy

$\square$ Somewhat difficult

$\square$ Very difficult

4. For you to understand the meaning of the labels and buttons in PPADS is:

$\square$ Very easy

$\square$ Moderately easy

$\square$ Somewhat difficult

$\square$ Very difficult

5. Using PPADS to perform the assigned tasks is: 
$\square$ Always easy

$\square$ Mostly easy

$\square$ Sometimes easy

$\square$ Never easy

6. The amount of information used in PPADS is:

$\square$ Too much information

$\square$ Just right

$\square$ Too little information

7. The length of decision support form is:
$\square$ Too long
$\square$ Just right
$\square$ Too short

8. The terms used in PPADS are:
$\square$ Always easily understood
$\square$ Moderately easily understood
$\square$ Sometimes easily understood
$\square$ Never easily understood

9. Please mark how much you agree or disagree with the following statements:

\begin{tabular}{|c|c|c|c|c|}
\hline & $\begin{array}{c}\text { Strongly } \\
\text { Disagree } \\
1\end{array}$ & $\begin{array}{c}\text { Disagree } \\
2\end{array}$ & $\begin{array}{c}\text { Agree } \\
3\end{array}$ & $\begin{array}{c}\text { Strongly } \\
\text { Agree } \\
4\end{array}$ \\
\hline $\begin{array}{l}\text { 1. I would find it easy to learn to us } \\
\text { the tool }\end{array}$ & & & & \\
\hline 2. Using the tool would not add stre & 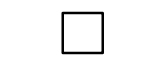 & & & - \\
\hline $\begin{array}{l}\text { 3. I was able to find the information } \\
\text { was looking for }\end{array}$ & & & & \\
\hline $\begin{array}{l}\text { 4. I found I could navigate the tool } \\
\text { quickly }\end{array}$ & & & & \\
\hline
\end{tabular}

10. Please mark how much you agree or disagree with the following statements: 


\begin{tabular}{|c|c|c|c|c|}
\hline & $\begin{array}{c}\text { Strongly } \\
\text { Disagree } \\
1 \\
\end{array}$ & $\begin{array}{c}\text { Disagree } \\
2 \\
\end{array}$ & $\begin{array}{c}\text { Agree } \\
3 \\
\end{array}$ & $\begin{array}{c}\text { Strongly } \\
\text { Agree } \\
4 \\
\end{array}$ \\
\hline $\begin{array}{l}\text { 1. The list of the neonate's current medical } \\
\text { condition was useful }\end{array}$ & $\square$ & $\square$ & $\square$ & $\square$ \\
\hline $\begin{array}{l}\text { 2. The list of neonate's current medical } \\
\text { conditions was easy to understand }\end{array}$ & $\square$ & $\square$ & $\square$ & $\square$ \\
\hline $\begin{array}{l}\text { 3. The list of the neonate's current } \\
\text { treatments was useful }\end{array}$ & $\square$ & $\square$ & $\square$ & $\square$ \\
\hline $\begin{array}{l}\text { 4. The list of the neonate's current } \\
\text { treatments was easy to understand }\end{array}$ & $\square$ & $\square$ & $\square$ & $\square$ \\
\hline $\begin{array}{l}\text { 5. The neonate's risk predictions were } \\
\text { useful }\end{array}$ & $\square$ & $\square$ & $\square$ & $\square$ \\
\hline $\begin{array}{l}\text { 6. The neonate's risk predictions were } \\
\text { easy to understand }\end{array}$ & $\square$ & $\square$ & $\square$ & $\square$ \\
\hline $\begin{array}{l}\text { 7. The reason a change in direction of care } \\
\text { was suggested was useful }\end{array}$ & $\square$ & $\square$ & $\square$ & $\square$ \\
\hline $\begin{array}{l}\text { 8. The reason a change in direction of care } \\
\text { was suggested was easy to understand }\end{array}$ & $\square$ & $\square$ & $\square$ & $\square$ \\
\hline 9. The decision support form was useful & $\square$ & $\square$ & $\square$ & $\square$ \\
\hline $\begin{array}{l}\text { 10. The decision support form was easy to } \\
\text { understand }\end{array}$ & $\square$ & $\square$ & $\square$ & $\square$ \\
\hline $\begin{array}{l}\text { 11. The information about the different } \\
\text { directions of care were presented in a } \\
\text { balanced way }\end{array}$ & $\square$ & $\square$ & $\square$ & $\square$ \\
\hline $\begin{array}{l}\text { 12. The glossary descriptions provided for } \\
\text { the different medical terms were useful }\end{array}$ & $\square$ & $\square$ & $\square$ & $\square$ \\
\hline $\begin{array}{l}\text { 13. The glossary descriptions provided for } \\
\text { the different medical terms were easy to } \\
\text { understand }\end{array}$ & $\square$ & $\square$ & $\square$ & $\square$ \\
\hline
\end{tabular}




\begin{tabular}{|c|c|c|c|c|}
\hline & $\begin{array}{c}\text { Strongly } \\
\text { Disagree } \\
1\end{array}$ & $\begin{array}{c}\text { Disagree } \\
\text { Agree }\end{array}$ & $\begin{array}{c}\text { Strongly } \\
\text { Agree } \\
4\end{array}$ \\
\hline $\begin{array}{c}\text { 14.After using the PPADS tool I would feel } \\
\text { more informed }\end{array}$ & $\square$ & $\square$ & $\square$ & $\square$ \\
\hline $\begin{array}{c}\text { 15.After using the PPADS tool I would } \\
\text { have a better understanding of the } \\
\text { direction of care options }\end{array}$ & $\square$ & $\square$ & $\square$ & $\square$ \\
\hline $\begin{array}{c}\text { 16.After using the PPADS tool I would feel } \\
\text { more certain about my decision }\end{array}$ & $\square$ & $\square$ & $\square$ & $\square$ \\
\hline $\begin{array}{c}\text { 17. After using the PPADS tool I would } \\
\text { feel clearer about how my values affect } \\
\text { my decision }\end{array}$ & $\square$ & $\square$ & $\square$ & $\square$ \\
\hline $\begin{array}{c}\text { 18.If given the opportunity I would use the } \\
\text { tool }\end{array}$ & $\square$ & $\square$ & $\square$ & $\square$ \\
\hline
\end{tabular}

Thank you very much for your participation. Please list any other comments including: overall opinion of the tool, positives, negatives, problems you had, and suggestions for improvements below: 